\title{
Zambia: Second Review Under the Three-Year Arrangement Under the Poverty Reduction and Growth Facility and Request for Waiver and Modification of Performance Criteria, and Financing Assurances Review-Staff Report; Staff Statement; Press Release on the Executive Board Discussion; and Statement by the Executive Director for Zambia
}

In the context of the second review under the three-year arrangement under the Poverty Reduction and Growth Facility and request for waiver and modification of performance criteria, and financing assurances review with Zambia, the following documents have been released and are included in this package:

- $\quad$ the staff report for the second review under the three-year arrangement under the Poverty Reduction and Growth Facility and request for waiver and modification of performance criteria, and financing assurances review, prepared by a staff team of the IMF, following discussions that ended on February 9, 2005, with the officials of Zambia on economic developments and policies. Based on information available at the time of these discussions, the staff report was completed on March 25, 2005. The views expressed in the staff report are those of the staff team and do not necessarily reflect the views of the Executive Board of the IMF.

- $\quad$ a staff statement of April 8, 2005 updating information on recent developments.

- $\quad$ a press release summarizing the views of the Executive Board as expressed during its April 8, 2005 discussion of the staff report that completed the review and request.

- $\quad$ a statement by the Executive Director for Zambia.

The documents listed below have been or will be separately released.

Letter of Intent sent to the IMF by the authorities of Zambia* Memorandum of Economic and Financial Policies by the authorities of Zambia* Technical Memorandum of Understanding* Enhanced Initiative for Heavily Indebted Poor Countries - Completion Point Document *May also be included in Staff Report

The policy of publication of staff reports and other documents allows for the deletion of market-sensitive information.

To assist the IMF in evaluating the publication policy, reader comments are invited and may be sent by e-mail to publicationpolicy@imf.org.

Copies of this report are available to the public from

International Monetary Fund • Publication Services

$70019^{\text {th }}$ Street, N.W. • Washington, D.C. 20431

Telephone: (202) 623-7430 • Telefax: (202) 623-7201

E-mail: publications@imf.org •Internet: http://www.imf.org

Price: $\$ 15.00$ a copy

\section{International Monetary Fund Washington, D.C.}



INTERNATIONAL MONETARY FUND

ZAMBIA

\title{
Second Review Under the Three-Year Arrangement \\ Under the Poverty Reduction and Growth Facility and Request for Waiver and Modification of Performance Criteria, and Financing Assurances Review
}

\author{
Prepared by the African Department \\ (In consultation with other departments) \\ Approved by Saul Lizondo and G. Russell Kincaid
}

March 25, 2005

- Discussions on the second review of the three-year Poverty Reduction and Growth Facility (PRGF) arrangement were held in Zambia from January 31 to February 9, 2005. The staff team met with Mr. Magande, Minister of Finance and National Planning; Mr. Fundanga, Governor of the Bank of Zambia (BoZ); other senior officials; representatives of the private sector; and members of the diplomatic and donor community.

- The staff team comprised Mr. Andrews (head), Mr. Dunn, Mr. Fassina (all AFR), Mr. Arnason (PDR), Mr. Baldini (FAD), and Mr. Kakoza (Resident Representative). The mission worked closely with World Bank staff.

- This report covers the second review under the PRGF arrangement. A Joint Staff Advisory Note on the authorities' Poverty Reduction Strategy Paper annual progress report and its addendum, including a supplement on objectives and policies for 2005, as well as the enhanced HIPC Initiative completion point document have been issued separately.

- Zambia is requesting the third disbursement under the current PRGF arrangement of SDR 5.502 million (1.1 percent of quota) to support the program outlined in the attached letter of intent (LOI) and memorandum of economic and financial policies (MEFP). The disbursement would be available upon the completion of the second review under the arrangement. The authorities have also requested waivers for one quantitative and one structural performance criterion. 


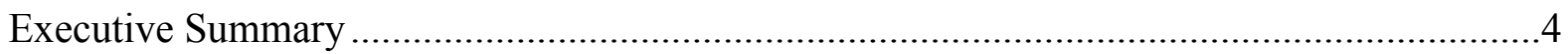

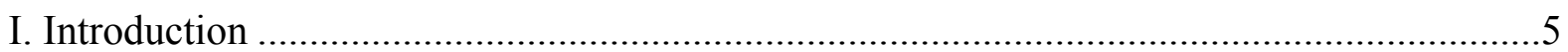

II. Recent Economic Developments and Performance under the PRGF-Supported Program...5

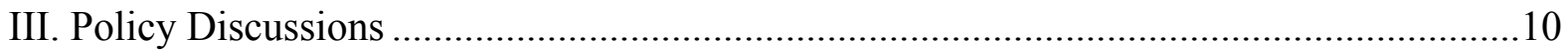

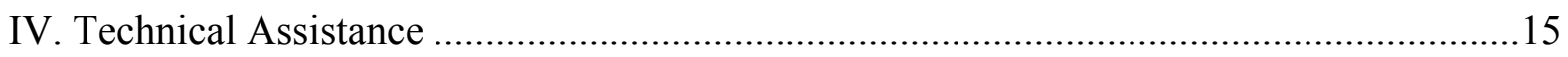

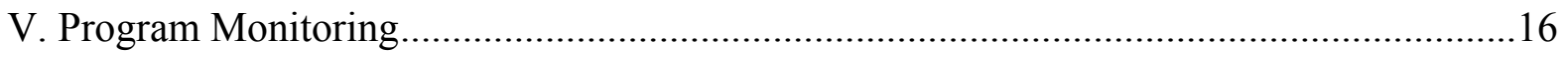

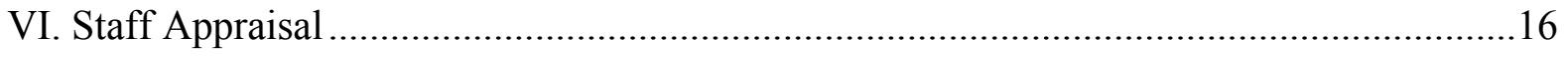

Figures

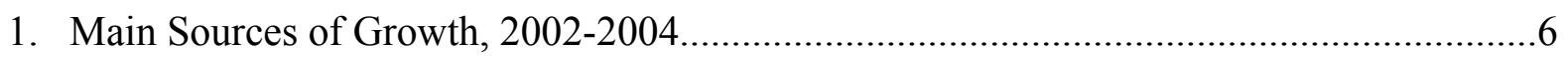

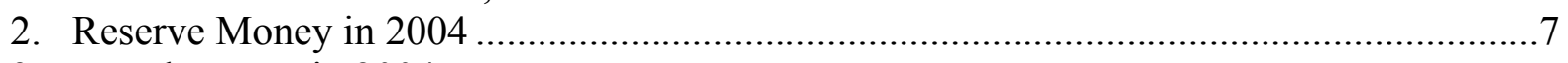

3. Broad Money in 2004 ................................................................................................

4. T-Bill Rates and Average Lending Rates of Commercial Banks,

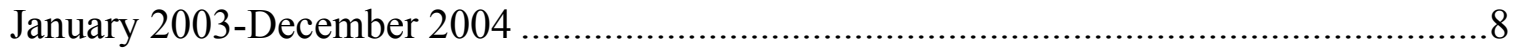

5. Effective Exchange Rates, January 2000-December 2004 ................................................ 8

6. Bilateral Exchange Rates, January 2000-December 2004 ...................................................

Box

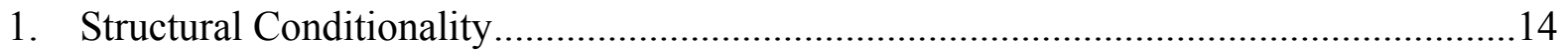

Tables

1. Selected Economic and Financial Indicators, 2002-07................................................18

2. Central Government Overall Operations, 2003-07 (in billions of kwacha).......................19

3. Central Government Overall Operations, 2003-07 (in percent of GDP) ...........................20

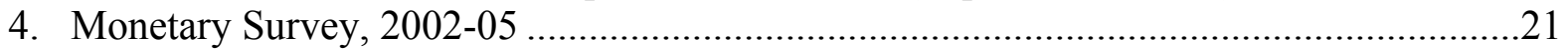

5. Assets and Liabilities of the Bank of Zambia, 2002-05 ..................................................23

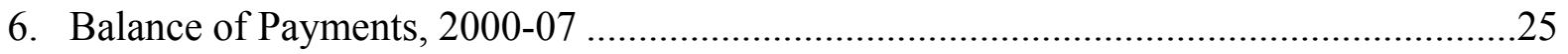

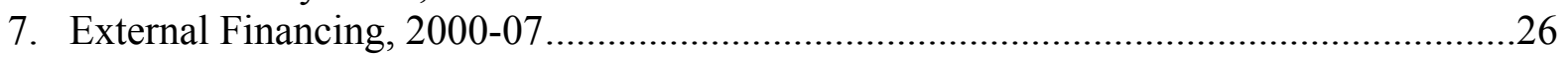

8. Schedule of Disbursements Under the PRGF Arrangement, 2004-07 .............................27 
Appendices

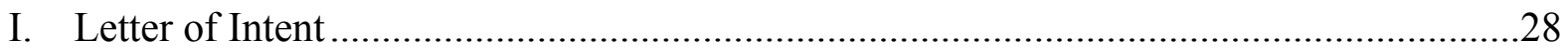

Memorandum of Economic and Financial Policies of the Government of Zambia ....30

Technical Memorandum of Understanding for the PRGF Arrangement....................44

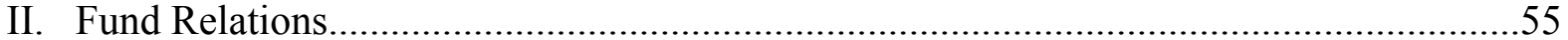

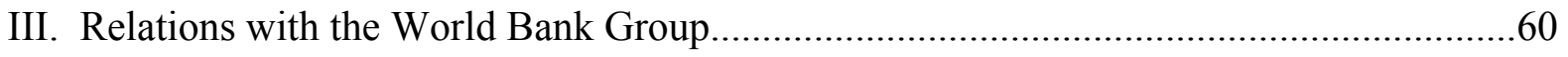




\section{Executive Summary}

\section{Recent Economic Developments and Performance Under the PRGF-Supported Program}

- Zambia's economy outperformed expectations in 2004: real GDP growth reached 5 percent, marking the fifth consecutive year of solid expansion, while inflation was held to $171 / 2$ percent (compared with the program projection of 20 percent), despite higher fuel prices.

- A strong fiscal adjustment and a consequent reduction in government domestic borrowing contributed to this positive outturn, easing pressure on inflation and interest rates. For the first time, budget allocations for priority poverty-reducing programs were fully released in 2004 .

- Performance under the PRGF-supported program has been good, although there were delays in some structural measures. All but one of the performance criteria (PCs) and all benchmarks under the quantitative program for December 2004 were observed. Two structural PCs were observed, and the third one, for end-February 2005, is now a prior action for the completion of the second review.

\section{Medium-Term Outlook and Program}

- The medium-term strategy in the PRSP focuses on reducing poverty through strong economic growth and diversification driven by the private sector, and a reorientation of government spending to priority areas. The strategy targets real GDP growth of at least 5 percent a year, single-digit inflation by 2007, and a strengthened international reserve position. Macroeconomic policies are anchored in limiting government's debt financing. Spending on poverty-reducing programs is expected to increase by 11/2 percentage points of GDP by 2007 .

\section{Program for 2005}

- The proposed program for 2005 aims to maintain the key features of the program approved by Executive Directors at the time of the first review under the PRGF. It envisages real GDP growth of 5 percent; an end-year inflation rate of no more than 15 percent; and a buildup of gross international reserves to 1.3 months of import cover by end- 2005 .

- Similarly, the program continues to limit government domestic borrowing to 1.6 percent of GDP, and includes a tightening of monetary policy. The authorities have requested a modification of quantitative targets for the first half of 2005 to reflect actions started in January 2005 to mop up the unanticipated expansion in liquidity at end-2004 and have proposed quarterly program targets for the second half of 2005.

- New structural measures are proposed for (i) the public expenditure management and financial accountability program; and (ii) the financial sector development plan.

- The program is subject to a number of risks. Attainment of the completion point and the approach of the elections in 2006 may lead to pressures for a relaxation of fiscal policy and a slowing of the momentum of reform. The staff therefore welcomes the emphasis in the government's recent statements, including the budget speech, on steadfast policy implementation. 


\section{INTRODUCTION}

1. On December 15, 2004, the Executive Board completed the first review under the PRGF arrangement with Zambia. Directors welcomed Zambia's improved economic performance and commended the authorities for a substantial fiscal adjustment that eased pressures on inflation and interest rates. Directors supported the proposed program for 2005, including a reduction in government's domestic borrowing and a tightening of monetary policy, and looked forward to further progress in reforms of the financial sector, private sector development and public expenditure management. Directors stressed the importance of increasing poverty-reducing expenditures over the medium term.

2. In the attached letter of intent (Appendix I), Zambia's Minister of Finance and National Planning (MoFNP) requests the third disbursement under the PRGF arrangement of SDR 5.502 million (1.1 percent of quota). This will be available on completion of the second review, which would also establish the minimum six months of satisfactory performance under a PRGF arrangement required immediately before reaching the completion point under the enhanced HIPC Initiative in view of an earlier interruption in Zambia's PRGF-supported program. In the attached memorandum of economic and financial policies (MEFP), the authorities describe performance under the program and propose modifications to quantitative targets ${ }^{1}$ and new structural measures for 2005 . The authorities also request waivers for nonobservance of one quantitative and one structural performance criterion.

\section{Recent ECONOMic DeVelopments And Performance Under the PRGF- SUPPORTED PROGRAM}

3. Economic growth of 5 percent in 2004 surpassed the 3.5 percent originally envisaged in the program, and inflation was held below the program projection. GDP growth in 2004 was broad-based (Table 1 and Figure 1). Mining output and construction activity - supported by strong private investment - grew by over 12 percent, while agriculture and manufacturing grew by more than 5 percent. Despite higher-than-expected fuel prices, inflation was 17.5 percent at end-2004, compared with the program projection of 20 percent.

4. Macroeconomic performance was aided by a large fiscal adjustment, which reduced pressures on interest rates and inflation. The overall fiscal deficit declined from 6.6 percent of GDP in 2003 to 1.7 percent in 2004, reducing the need for domestic borrowing from 5.1 percent of GDP to 0.8 percent of GDP over the same period (Tables 2 and 3 ). Total

\footnotetext{
${ }^{1}$ The modifications are described in paragraph 24 on program monitoring.
} 


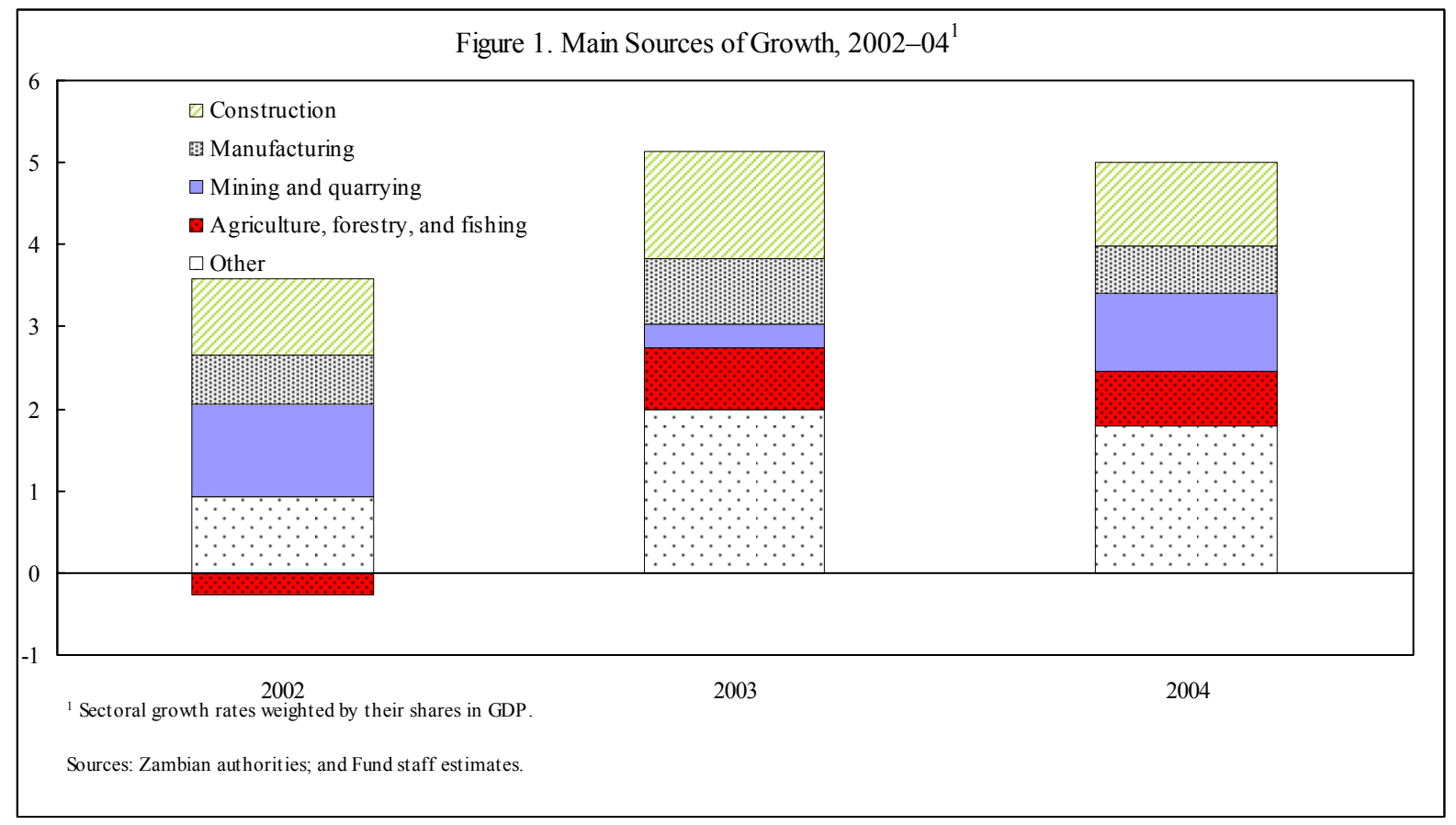

revenues were 0.3 percent of GDP lower than programmed, mainly because of reduced tax collections from commercial banks and earlier payment of value-added tax (VAT) refunds. Domestically financed spending (including interest payments) was 0.2 percent of GDP lower than programmed. The wage bill declined as budgeted because the government granted no nominal wage increase in 2004 and curtailed housing allowances. Recurrent departmental charges were 0.5 percent of GDP higher than originally budgeted reflecting supplementary allocations for the military and allocations to settle arrears to the electricity utility, ZESCO. ${ }^{2}$ Lower interest payments and cutbacks on nonpriority capital projects offset these expenditures. At the same time, the government fully funded expenditures on priority poverty-reducing programs (PRPs). However, authorized payments of about 1 percent of GDP were not encashed, mainly because more than half of the budget support for 2004 came in late December. The bulk of these releases that were not encashed are expected to lapse because ministries did not enter into corresponding spending commitments by the end of 2004. However, the staff and the authorities estimate that up to one-third of these releases not encashed in 2004 are associated with spending commitments and will therefore be encashed in 2005 and reflected in higher domestic financing in 2005.

\footnotetext{
${ }^{2}$ These payments were called for in the strategy for ZESCO, which, among other measures, requires government's financial relations with ZESCO to be commercially based.
} 
5. Large unanticipated swings in liquidity continued to complicate the implementation of monetary policy. In the first half of 2004, intervention by the BoZ was not strong enough to offset the decline in reserve requirements at the end of 2003 . The BoZ began to tighten liquidity in mid2004, and the growth in reserve and broad money slowed (Figures 2 and 3 ). Nevertheless, at the time of the first review monetary aggregates were seen as likely to be higher than projected for 2004. In addition, at the end of the year, large foreign currency deposits by corporations into commercial bank accounts and, to a lesser extent, disbursements of donor support and related transfers to line-ministries' bank accounts (as noted above) pushed up the 12-month increase in reserve and broad money to 21 percent and 30 percent, respectively (Tables 4 and 5). ${ }^{3}$ However, in January 2005, the BoZ intervened heavily through open market operations, reducing the 12month growth in reserve money to 14 percent; at the same time, the 12month increase in broad money declined to 25 percent.
Figure 2. Reserve Money in 2004

(Cumulative change from December 2003; in percent)

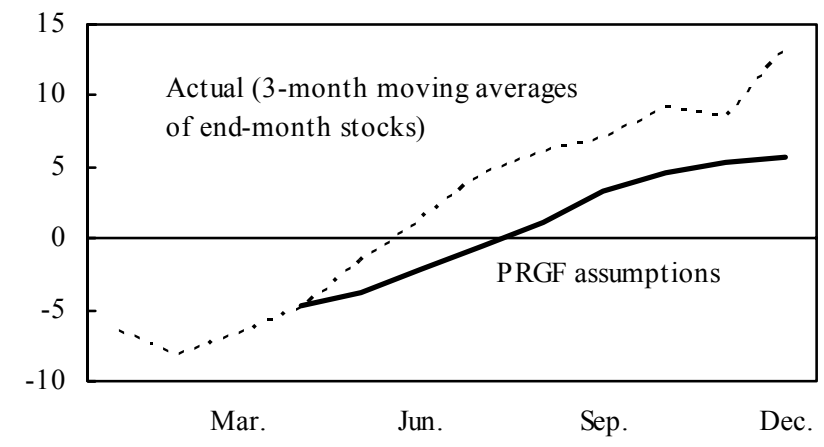

Sources: Zambian authorities; and Fund staff estimates.

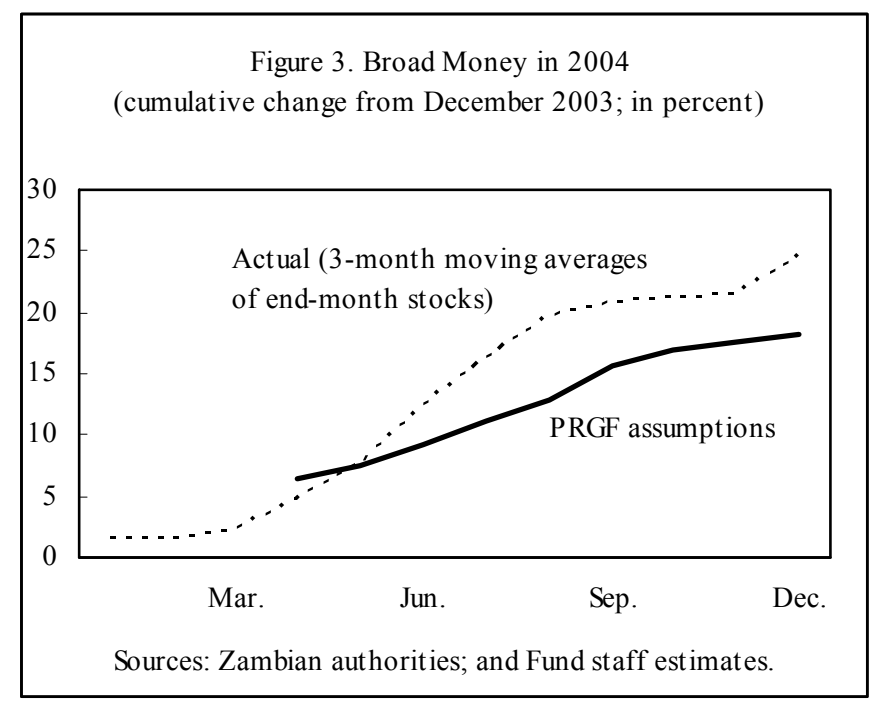

\footnotetext{
${ }^{3}$ Mainly due to higher corporate foreign currency deposits, commercial banks' net foreign assets rose by almost US\$50 million in December 2004. The domestic currency component of broad money increased by 19 percent in the 12 months to December 2004, broadly in line with the program.
} 
6. Despite these developments, monetary policy-supported by the fiscal adjustment - was effective in reducing pressure on commercial banks' lending rates. Interest rates on treasury bills - which had fallen from over 30 percent in December 2003 to about 7 percent in June 2004, following the reduction in reserve requirements - began to rise and stabilized in the final quarter at about 18 percent. Commercial bank lending rates did not fall as sharply as treasury bill yields and remained at around 37 percent in the second half of 2004 (Figure 4). Lower government borrowing facilitated an expansion in credit to the private sector of almost 50 percent in 2004, with agriculture and manufacturing accounting for the bulk of this rise.

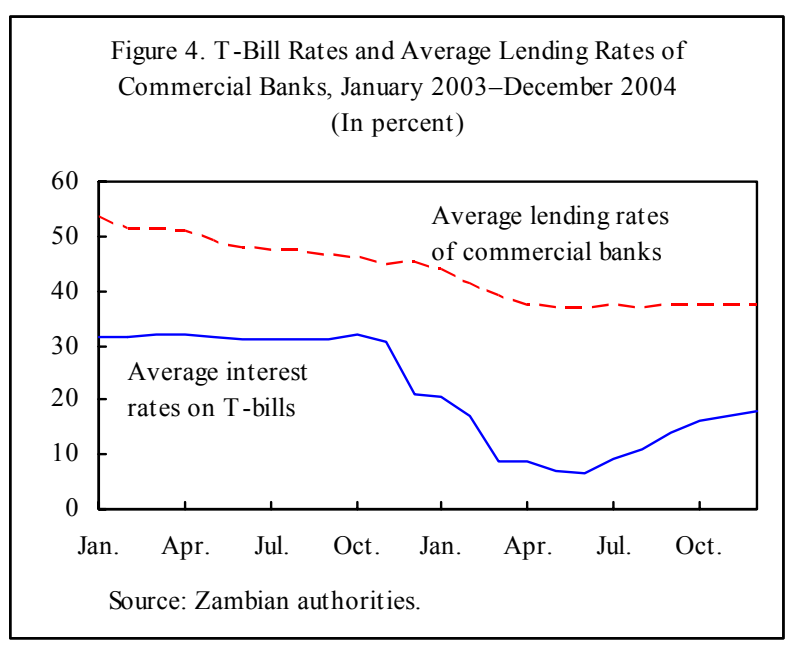

7. Zambia's external position strengthened in 2004. Metal exports increased by 65 percent, reflecting higher production and a 50 percent increase in copper prices. The growth of nonmetal exports also exceeded expectations, and, despite large capital imports in the mining sector and high oil prices, the current account deficit (including grants) narrowed from 7.5 percent of GDP in 2003 to 4.8 percent in 2004 (Table 6). Gross international reserves increased more than programmed (by US $\$ 35$ million) to 1.2 months of imports (Table 7). The kwacha was broadly stable against the U.S. dollar but appreciated in real effective terms by about 8 percent in 2004 (Figures 5 and 6).
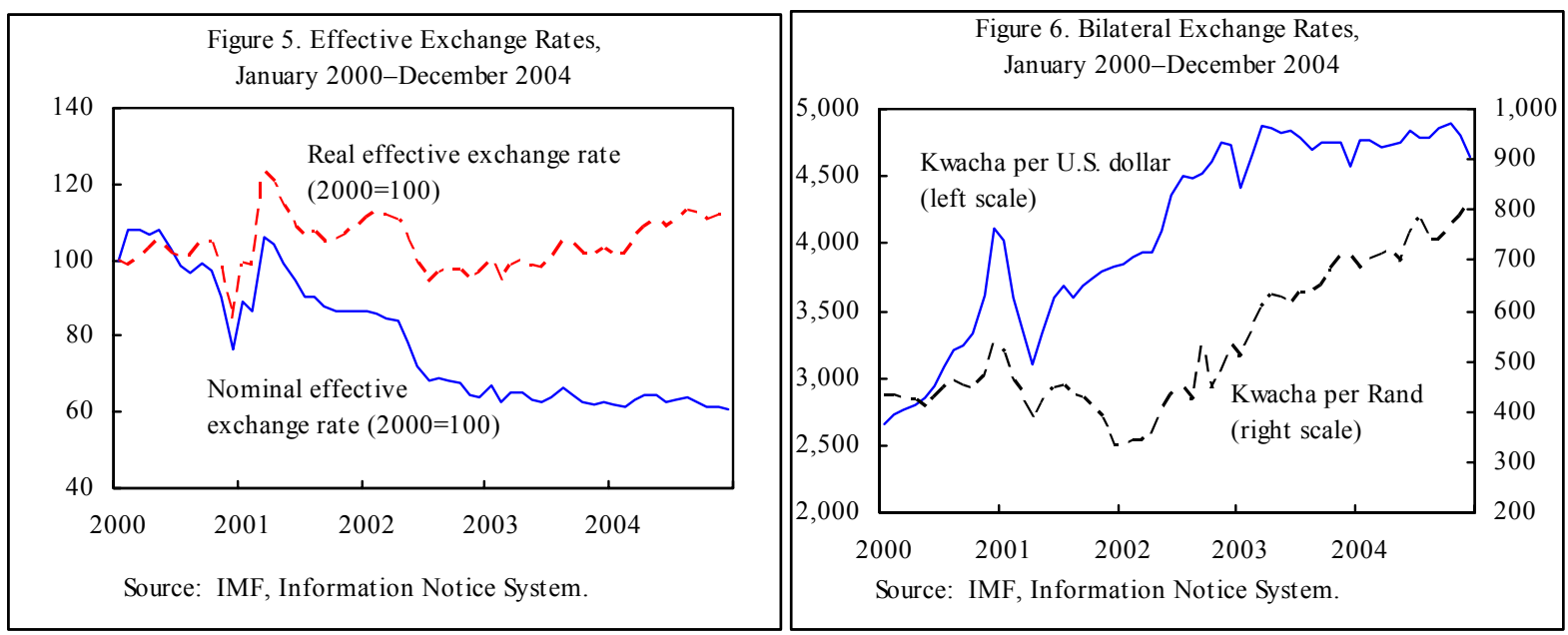


\section{Performance under the program continued to be strong, although some} structural reforms were delayed. All but one quantitative performance criteria and all quantitative benchmarks for December were observed, some by wide margins. The authorities are requesting a waiver because of a delay in debt-service payments to Russia, which have now been made. ${ }^{4}$ Regarding structural conditionality, the performance criterion for January 2005 on the adoption of the work program for the public expenditure management and financial accountability (PEMFA) reforms was met. The performance criterion for February 2005 on cabinet approval of legislative proposals to strengthen the BoZ's supervision of nonbank financial institutions (NBFIs), which were developed with technical assistance from the Fund, was not met. The government is requesting a waiver for the nonobservance of this performance criterion based upon its subsequent implementation, which is now a prior action for completion of the review. This action has not yet been taken and the Board will be informed separately. The government has continued to refrain from making extrabudgetary payments for which it is not legally liable (a continuous performance criterion) and has observed limits on funding unforeseen expenditures (a continuous benchmark). Quarterly budget execution reports have been published, but with a delay of more than 45 days. The government developed, in a consultation with stakeholders, a wider definition of poverty-reducing spending that was used in the preparation of the 2005 budget (a program benchmark). ${ }^{5}$ Further consultations with stakeholders may result in some refinement of this classification.

\footnotetext{
${ }^{4}$ The payments in question fell due in October 2004, but a waiver was not requested at the completion of the first review in December. Based on a communication to the Russian government from the Zambia authorities indicating (i) that the payment had not been made because of a misunderstanding over the due date; and (ii) that a payment instruction had been issued, AFR concluded that the late payment did not constitute a breach of the performance criterion on the nonaccumulation of arrears. However, after the completion of the review, the payment instruction was not executed, while the Zambian authorities sought to verify that interest had not been overpaid. The authorities did not inform the staff of this further delay and did not contact the creditor who, in early January 2005, informed the Paris Club that the payment was in arrears. LEG has reviewed the facts in this case and concluded that the continuous performance criterion was not observed. However, the nonobservance did not give rise to a noncomplying disbursement in December because the Zambian authorities had the right to rely on the staff's assessment at the time that the PC had been met and no waiver was required. Although the payments have now been made, a waiver is needed for the second review because of the continuous nature of the PC.

${ }^{5}$ This new definition and its rationale are described the Progress Report on PSRP Implementation of February 2005 (Appendix II of the Addendum to the Second PRSP Implementation Progress Report, 2004). As indicated in the JSAN (3/11/05) (www.imf.org), the definition of poverty reducing expenditures was expanded to cover all public expenditures related to PRSP implementation.
} 


\section{Policy Discussions}

9. Against the background of the strong fiscal adjustment in 2004, discussions focused on consolidating these gains and maintaining the momentum of growth. The government reaffirmed the strategy set out in its PRSP to promote growth through diversification and creation of an enabling environment for the private sector, while improving public services. The central macroeconomic objectives over the medium term continue to be growth of at least 5 percent a year, a gradual reduction in inflation to a single

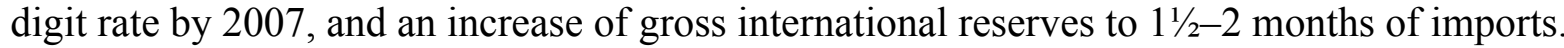
The framework is anchored on limiting the government's debt financing and seeks to reorient spending toward poverty reduction.

10. As indicated at the time of the first review under the PRGF arrangement, the medium-term expenditure framework envisages large spending for elections and the constitutional review. A technical working group headed by the Ministry of Justice with representatives from the Electoral Commission of Zambia and the donor community is examining these expenditure estimates, drawing on international experience. Election-related expenditures in 2005 are fully-funded in the budget, and the government reiterated its intention to close financing gaps in 2006 and 2007 through a combination of additional grants and offsetting measures.

11. The government's macroeconomic framework for $\mathbf{2 0 0 5}$ remains essentially as presented in the November MEFP (Table 1). However, the new MEFP envisages a tightening of the monetary program to reverse the sharp increase in monetary aggregates in late 2004. The MEFP also proposes program targets for the second half of 2005 and new structural measures in public expenditure management, external debt management, and financial sector development.

12. The authorities and the staff confirmed that the budget submitted to parliament in late January is consistent with the framework presented to the Board at the first review in December 2004. The budget retains the objective of limiting government's domestic borrowing in 2005 to 1.6 percent of $\mathrm{GDP}^{6}$ and the domestic primary surplus of 0.9 percent of GDP is also in line with the framework of the first review (Tables 2 and 3 ). The overall deficit, including grants, is now projected to be 2.5 percent of GDP or 0.3 percentage points higher than envisaged at the first review. This increase reflects a small shift in the composition of external financing from grants to highly concessional loans. Recourse to external borrowing (as a percent of GDP) in 2004-05 would, however, be as

\footnotetext{
${ }^{6}$ The program includes an adjustor to accommodate the possible effect on domestic financing in 2005 of expenditures that were authorized, but not spent in 2004. Full use of the adjustor would entail additional domestic financing of 0.3 percent of GDP (see paragraph 4) but domestic financing in 2004 and 2005 combined would still be lower than originally programmed because of the strong outturn in 2004. The stock of domestic debt would decline to 16.9 percent of GDP at end-2005 compared with the 17.7 percent of GDP projected at the time of the first review.
} 
originally envisaged. The budget includes broadly revenue-neutral tax measures. Revenue losses totaling 0.2 percent of GDP will result from bringing some agricultural exports into the VAT regime, raising the threshold for income tax, and lowering the top rate of income tax. These losses will be more than offset by the expiration of Konkola Copper Mines' duty exception on imports of consumer goods and by the introduction of export taxes to replace bans on timber and scrap metal exports (see paragraph 11 of the MEFP). On the expenditure side, a small increase in the wage bill to hire additional teachers is offset by cuts outside the education budget, including cancellation of a census that was planned as part of the constitutional review process. The budget allocates about 1 percent of GDP to settle arrears, with the bulk of this for clearance of arrears on housing allowances and pensions. ${ }^{7}$

\section{The budget was prepared using a new, wider, definition of poverty-reducing} expenditures, as called for by a program benchmark. ${ }^{8}$ A comparison with the 2004 outturn using the same classification, which should be available within the next two months, is expected to show a significant rise in poverty-reducing expenditures in 2005, reflecting the net hiring of teachers and health workers (see paragraph 12 of the MEFP). While uncertainties remain, the recruitment provided for in the budget is also expected to absorb available trained staff in 2005 . The authorities reiterated their commitment to increase poverty-reducing expenditures over the medium term.

\section{Within this framework, discussions focused on the need to ensure effective} budget execution. In addition to accommodating net hiring, the budget includes an allocation for a general wage increase. The staff agreed that announcement of this envelope - and the lifting of the nominal wage freeze applied in 2004 - would facilitate negotiations with public employees' unions. A negotiating team has been established with MoFNP representation. The authorities reaffirmed their commitment to hold the wage bill, including housing allowances within the budgeted amount. The authorities also stressed the need for tight control of expenditures.

\footnotetext{
${ }^{7}$ External commercial arrears, for which a financing assurances review is required, have been the subject of good faith negotiations between the authorities and the creditors concerned, which are continuing.

${ }^{8}$ Under the new definition, poverty-reducing expenditures account for 36 percent of the 2005 budget spending (including on external debt amortization) or about 10 percent of GDP. However, this total includes donor-financed expenditures not under government control. Poverty-reducing spending accounts for 22.3 percent of domestically financed expenditure (or 5 percent of GDP). Activity-based budgeting has also improved the classification of capital and recurrent programs. In the 2005 budget, recurrent departmental charges are 1.6 percent of GDP higher than in the November MEFP, and transfers and pensions are 1.9 percent of GDP higher, while capital spending and the residual category of other recurrent expenditures have declined by slightly more than these amounts.
} 


\section{The discussion of monetary policy for $\mathbf{2 0 0 5}$ took place against the backdrop of} the sharp increases in monetary aggregates in December. The staff agreed with the authorities that exceptional factors had contributed to the surge in reserve and broad money in December. However, monetary expansion was already higher than envisaged before these factors intervened so that monetary growth targets were consistently exceeded. It was therefore agreed that efforts that began in January to reverse the buildup in liquidity are to continue in line with a revised monetary program, which will retain reserve and broad money stock targets for end-2005 established at the first review (Tables 4 and 5). Monetary tightening will be facilitated by the smaller increase in gross international reserves in 2005 that is now targeted, taking into account the over-performance in 2004. In addition, greater stability in the money multiplier - now that changes in reserve requirements have worked through the system - is expected to improve the control of broad money. The authorities recognize that close coordination of monetary and fiscal policy will also be essential to control monetary aggregates while avoiding undue pressures on interest rates or the exchange rate. Particular attention will be paid to the possible drawdown of government deposits to fund expenditures that were authorized but not executed in 2004. In the event that government's domestic borrowing in the early part of 2005 is higher as a result of the execution of expenditures authorized last year - as allowed for by the corresponding program adjustor - the implications for the monetary program will need to be assessed. The authorities also recognized the need to widen the range of instruments and expand the resources available to deal with unexpected inflows of liquidity, issues that will be addressed in MFD's technical assistance to the BoZ.

16. External prospects for $\mathbf{2 0 0 5}$ remain favorable. A further rise in copper output should offset a projected easing of prices, and nonmetal exports are expected to expand briskly. Thus, although imports will rise with economic activity, the current account deficit (including grants) is projected to narrow further to 4.3 percent of GDP (Table 6). The authorities now project external debt service in 2005 to be higher than at the time of the first review, but this is expected to be offset by an increase in external budget support. Including debt-service relief following the attainment of the HIPC Initiative completion point, the 2005 program is fully financed (Table 7).

17. The authorities agreed that, in the area of structural reforms, they should give priority to key steps supporting the implementation of the PEMFA program as well as areas in which preparatory work is already well advanced. ${ }^{9}$ The framework for monitoring and evaluating the PEMFA program, to be used in the first assessment of the program later this year, is expected to draw upon the public expenditure and financial accountability (PEFA) framework and the benchmarks of the HIPC Initiative assessment and action plan to track poverty-reducing spending. ${ }^{10}$ Initial PEMFA reforms monitored under

\footnotetext{
${ }^{9}$ See Box 1 for a fuller discussion of structural reforms and the roles of other institutions.

${ }^{10}$ The second "Public Expenditure Management Country Assessment and Action Plan: Zambia" was conducted in October 2003.
} 
the PRGF arrangement will include the development of new financial regulations and the preparation of a cash-flow framework for line-ministries. In addition, the MoFNP intends to bring forward the preparation of the medium-term expenditure framework (MTEF) for 200608; a Green Paper is to be published by end-August, two months earlier than in $2004 .{ }^{11}$ The government also expects to take additional steps to control defense spending after the auditor general has concluded special audits of the military wage bill and recurrent departmental charges (MEFP, paragraph 24).

\section{The authorities and staff agreed on the urgency of strengthening debt}

management capacity. ${ }^{12}$ The immediate priority is to routinely update the debt database to cover all of the government's external obligations. Thus, the stock of the government's external debt will be validated quarterly, beginning with the second quarter of 2005, and projections of debt service for the following three years (the time horizon of the MTEF) will be provided to the budget office. The government expects to upgrade its debt-management operations with technical support from the World Bank.

\section{The government continues to make progress in the resolution of the NBFIs}

(MEFP, paragraphs 26-27). The BoZ's close supervision of the National Savings and Credit Bank and the Development Bank of Zambia has yielded some improvements in their balance sheets, but further work is needed. Specifically, with technical assistance from cooperating partners, the authorities are focusing on the development of operational business plans leading to full compliance with the Banking and Financial Services Act and incorporation under the Companies Act in 2006, including limits on ownership concentration, which would also apply to the government's holdings. The BoZ also continues to closely monitor the operations of the Zambia National Building Society (ZNBS). In February, the BoZ received a revised business plan for the ZNBS. By end-April, the Government and the BoZ, in consultation with the staffs of the Fund and the World Bank, will adopt an action plan for the resolution of ZNBS.

\footnotetext{
${ }^{11}$ PEMFA reforms will also support the implementation of the integrated financial management information system (IFMIS). However, as noted in the HIPC completion point document (3/25/05) at www.imf.org, and paragraph 23 of the MEFP, factors beyond the government's control have again delayed the signing of a contract to supply hardware and software for the IFMIS.

${ }^{12}$ See Box 4, in the HIPC completion point document (3/25/05) at www.imf.org.
} 


\section{Box 1. Structural Conditionality}

\section{Coverage of Structural Conditionality in the PRGF-Supported Program for 2005}

The prior action, structural performance criteria, and benchmarks in the program for 2005 (Appendix I, Table 4) focus on areas critical for achieving the program's macroeconomic objectives and also include measures that were not implemented in 2004. The conditions cover:

- Public expenditure management: development of an assessment framework for the PEMFA program and review of the implementation; budget preparation (MTEF), execution, and reporting (publication of quarterly reports); piloting of an IFMIS and conducting a preliminary review of this pilot.

- Debt management: quarterly validation of external debt data and updating of the debt-service schedule.

- Financial sector: cabinet approval of proposals to strengthen the BOZ's supervision of NBFIs; progress on the resolution of three NBFIs; issuance of a new invitation for bids for purchase of 49 percent equity in $\mathrm{ZNCB}$, with management control.

- Governance and transparency: unbudgeted expenditures funded only to the limits of the budget contingency or after cabinet approval, with offsetting savings identified.

World Bank's conditionality under an Economic Management and Growth Credit covers the following areas:

- Measures to prevent public sector arrears to public utilities.

- Initial reforms of public sector pension schemes.

- Resolution of non bank financial institutions.

- Public expenditure management: revision of the Finance Act and strengthening budget preparation and auditing.

- Control of the government payroll and consolidation of allowances into basic salaries.

The European Union's conditionality includes funding targets for the Ministry of Health, the Ministry of Education, the Auditor General Office, and poverty-reducing programs; reporting of budget execution; and progress in primary education, HIV/AIDS prevention, children and women's health, and female empowerment.

The African Development Bank's conditionality focuses on strengthening the autonomy and effectiveness of the Auditor General's Office.

Bilateral donors' conditionality is concentrated — in a joint initiative — on the PEMFA program. In addition, some donors are providing budget support conditioned on progress in macroeconomic stability, public financial management, core structural reforms, health, education, and HIV/AIDS. 
20. The Government reiterated its commitment to the sale of 49 percent of the equity in the Zambia National Commercial Bank (ZNCB) to a strategic investor. Following protracted and ultimately unsuccessful negotiations on this sale, in mid-March, the Zambia Privatization Agency (ZPA) terminated discussions with the preferred and reserve bidders (MEFP, paragraph 28). With the aim of expediting this process, the ZPA will issue a new invitation for bids for purchase of 49 percent of the equity in $\mathrm{ZNCB}$ with management control. In view of the importance of protecting the budget from further losses and improving the functioning of the financial system, it was agreed this action would constitute a performance criterion for the next review under the PRGF. Given the preliminary interest exhibited by some reputable banks operating in the region, it is expected that the selection of new bidders and ensuing negotiations would proceed at a rapid pace.

21. The BoZ also intends to implement other recommendations from the Financial Sector Development Plan. Revisions to the Banking and Financial Services Act (BFSA), to be prepared with technical assistance from MFD and LEG, are expected to address issues beyond the three insolvent NBFIs, with the aim of strengthening the BoZ's regulatory and supervisory powers and modernizing the regulation of the mortgage market. The authorities are also preparing amendments to the Bank of Zambia Act to address the weaknesses identified in the Safeguard Assessment concluded in October 2004 (see Appendix II).

22. Priorities and institutional arrangements are being established to support the implementation of the private sector development initiative (PSDI). In February, the Domestic Business Council (chaired by the President) and the PSDI steering committee met for the first time. These groups bring together government officials and representatives of the business and donor communities to monitor implementation of the PSDI. It is expected that development partners will also support this effort. Alongside these steps to establish an implementation mechanism, initial measures to be taken focus on revisions of the Trade Licensing Act, and amendment of the Investment Act to simplify investment approvals (paragraph 29 of the MEFP).

\section{Technical Assistance}

23. Technical assistance (TA) from the Fund will support program implementation. In the financial sector, TA from MFD and LEG will be provided to strengthen the BoZ's liquidity management, and amend the Bank of Zambia Act and legislation on the supervision of banks and other financial institutions. The authorities have requested support from FAD to prepare regulations and accounting manuals for the new Finance Act. Following the completion of the data module of the Report on Standards and Codes, support from STA will focus on bringing the fiscal accounts into line with the Government Finance Statistics Manual (2001), and on improving the monetary survey. The authorities have also expressed interest in TA to rebase the national accounts and the consumer price index. 


\section{Program Monitoring}

24. Progress in implementing the PRGF-supported program will continue to be monitored quarterly. Performance criteria are now proposed through the end of 2005. In addition, the government is requesting modifications to the performance criteria for March and June on the net domestic assets of the BoZ (to reflect the changes to the monetary program), the net domestic financing of the government (to reflect a minor rephasing of spending and the inclusion of the adjustor discussed above), and a modification to the quarterly ceilings on the central government wage bill (to accommodate additional hiring).

\section{Staff Appraisal}

25. The continued strengthening of Zambia's economic performance is most welcome. Growth in 2004 reached 5 percent, surpassing expectations, and the economy is projected to grow at least as rapidly in 2005, extending to six years the strongest and longest period of growth in more than two decades. Although faster growth is needed to reduce pervasive poverty, it is encouraging that the recent expansion has broadened, with agriculture growing steadily. Inflation remains too high, but important progress has been made, setting the stage for further declines in inflation over the next two years.

26. Improved macroeconomic management has underpinned this strengthened performance. In 2004, a much-needed fiscal adjustment halted an unsustainable rise in domestic debt, thereby reducing pressures on both prices and interest rates. The sharp decline in government borrowing was made possible by a fiscal policy that embraced politically difficult spending restraints, and by holding total spending and its composition much closer to the budget than in the past. These improvements were evident in the full and timely funding, for the first time, of priority poverty-reducing programs in 2004.

27. Appropriately, the budget for 2005 continues to restrain domestic borrowing. The staff notes that, as a result of the strong fiscal adjustment in 2004, the budget also provides for a significant increase in real spending in priority areas, including a welcome increase in resources to hire teachers and health workers. Within this framework, the staff urges the authorities to maintain fiscal discipline to enhance the credibility of the disinflation policy.

28. The staff welcomes the efforts that are now under way to examine the level and composition of expenditures for the elections and the constitutional review process through 2007. Progress in this area is needed to ensure that the next medium-term expenditure framework provides for these expenditures, while the projected financing gaps over the medium term are closed through cuts in nonpriority areas or additional external grants.

29. Good progress has been made in further defining PEMFA reforms including the adoption, in close cooperation with development partners, of a work program for the first year. The staff supports the initial emphasis placed on developing a monitoring and evaluation framework, which should focus on attaining measurable improvements in public expenditure management. At the same time, the staff urges the authorities to ensure the 
implementation of reforms covered under the PRGF-supported program. Overall, it is important that the PEMFA program carry forward the progress that has been achieved in strengthening public expenditure management and the preparations for implementation of the integrated financial management information system.

30. Recent experience has highlighted the importance of debt management. The staff welcomes the authorities' commitment to strengthen and centralize debt management in a single department in the MoFNP with the assistance of the World Bank. However, there is also an urgent need to ensure that, under the existing institutional structure, the external debt database is updated regularly so that the budget estimates capture all debt service due, including that on new disbursements and rescheduling agreements.

31. The Bank of Zambia has taken appropriate steps so far in 2005 to reverse the overly loose monetary policy in 2004, as well as the surge in liquidity that occurred at the end of 2004. Continued restraint will be required to ensure that monetary growth is contained. Close coordination of monetary and fiscal policy will be essential to ensure that the reserve money program is consistently implemented.

32. Reforms are needed to create an environment more conducive to private sector development. Implementation of the reform agenda is now key. The establishment of the domestic business council and a steering committee should support implementation; the initial focus should be on simplifying the regulatory and licensing framework.

33. The program is subject to a number of risks. The economy remains vulnerable to climatic and terms of trade shocks. In addition, attainment of the completion point under the HIPC Initiative and the approach of the elections in 2006 may lead to pressures for a relaxation of fiscal policy and a slowing of the momentum of reform. The staff therefore welcomes the emphasis in the government's recent statements, including the budget speech, on steadfast policy implementation, which is needed to maintain Zambia's improved economic performance and catalyze further donor support. At the same time, a more consistent implementation of the monetary program is needed to avert the risk of continued high inflation.

34. The staff recommends that Zambia be granted waivers for the nonobservance of the two performance criteria noted above and that the second review under the PRGF

arrangement be completed. The authorities have settled the external arrears that caused the nonobservance of one quantitative performance criterion. Moreover, cabinet approval of proposals to strengthen BoZ supervision of NBFIs justifies the waiver that is requested for the nonobservance of the corresponding structural performance criterion. The staff also recommends that the performance criteria be modified as requested and that the financing assurances review be completed. 
$-18-$

Table 1. Zambia: Selected Economic and Financial Indicators, 2002-07

\begin{tabular}{|c|c|c|c|c|c|c|c|c|}
\hline & \multirow[t]{2}{*}{2002} & \multirow[t]{2}{*}{2003} & \multicolumn{2}{|c|}{2004} & \multicolumn{2}{|c|}{2005} & \multirow{2}{*}{$\begin{array}{l}2006 \\
\text { Proj. }\end{array}$} & \multirow{2}{*}{$\begin{array}{l}2007 \\
\text { Proj. }\end{array}$} \\
\hline & & & Prog. & Prel. S & Rpt. 04 & Proj. & & \\
\hline & \multicolumn{8}{|c|}{ (Annual percentage change, unless otherwise indicated) } \\
\hline \multicolumn{9}{|l|}{ National income and prices } \\
\hline Real GDP & 3.3 & 5.1 & 3.5 & 5.0 & 5.0 & 5.0 & 5.0 & 5.0 \\
\hline GDP deflator & 19.9 & 19.8 & 19.6 & 20.0 & 15.5 & 15.2 & 9.3 & 6.0 \\
\hline Consumer prices (annual average) & 22.2 & 21.5 & 18.5 & 18.0 & 19.4 & 16.2 & 12.3 & 7.3 \\
\hline Consumer prices (end of period) & 26.7 & 17.2 & 20.0 & 17.5 & 15.0 & 15.0 & 10.0 & 5.0 \\
\hline \multicolumn{9}{|l|}{ External sector } \\
\hline U.S. dollar value of exports of goods and services & 2.4 & 15.7 & 21.5 & 49.4 & 7.3 & 8.6 & 3.9 & 1.5 \\
\hline U.S. dollar value of imports of goods and services & -2.5 & 13.3 & 13.7 & 23.3 & -1.1 & 2.8 & 9.2 & 0.5 \\
\hline Export volume (goods) & 11.2 & 1.8 & 4.9 & 5.6 & 21.7 & 19.5 & 17.9 & 7.7 \\
\hline Import volume (goods) & -3.9 & 6.9 & 7.7 & 4.3 & 0.1 & 7.4 & 17.3 & 3.1 \\
\hline Copper export volume & 11.3 & 7.1 & 8.0 & 8.7 & 22.0 & 22.0 & 14.8 & 4.5 \\
\hline Nonmetal export volume & 20.3 & -3.7 & 3.6 & 17.7 & 9.7 & 9.1 & 7.5 & 8.0 \\
\hline Copper export prices (average, U.S. dollars per pound) & 0.70 & 0.78 & 0.96 & 1.16 & 0.99 & 1.09 & 0.90 & 0.82 \\
\hline Nominal effective exchange rate (annual average) & -19.3 & -14.1 & -13.5 & -2.2 & $\ldots$ & $\ldots$ & $\ldots$ & $\ldots$ \\
\hline Real effective exchange rate (annual average) & -5.8 & -1.7 & -0.2 & 8.3 & $\ldots$ & $\ldots$ & $\ldots$ & $\ldots$ \\
\hline Terms of trade & -6.7 & 4.2 & 10.7 & 20.3 & -11.7 & -1.0 & -6.8 & -2.9 \\
\hline \multicolumn{9}{|l|}{ Money and credit (change in percent of beginning-of-year M2) } \\
\hline Net foreign assets & 68.5 & -9.0 & 0.0 & 13.9 & 24.7 & 19.5 & $\ldots$ & $\ldots$ \\
\hline Net domestic assets & -37.0 & 32.5 & 18.1 & 16.3 & -5.7 & -4.7 & $\ldots$ & $\ldots$ \\
\hline Net domestic credit & -29.4 & 36.6 & 18.8 & 16.4 & -5.7 & -4.7 & $\ldots$ & $\ldots$ \\
\hline Net claims on government & 3.1 & 27.0 & 9.7 & 1.0 & 6.1 & 5.9 & $\ldots$ & $\ldots$ \\
\hline Claims on nongovernment & 6.1 & 9.6 & 9.1 & 15.4 & 8.8 & 8.8 & $\ldots$ & $\ldots$ \\
\hline Broad money & 31.5 & 23.4 & 18.1 & 30.2 & 19.0 & 14.8 & $\ldots$ & $\ldots$ \\
\hline Velocity & 4.5 & 4.6 & 4.8 & 4.4 & 4.7 & 4.7 & $\ldots$ & $\cdots$ \\
\hline \multicolumn{9}{|l|}{ Central government budget } \\
\hline Revenue (excluding grants) & 16.0 & 26.5 & 30.0 & 28.8 & 21.9 & 21.1 & 15.0 & 11.2 \\
\hline Grants & 79.0 & 5.5 & -2.5 & 0.6 & 47.6 & 30.5 & 31.4 & 8.1 \\
\hline Expenditures 1/ & 20.7 & 24.6 & 10.2 & 9.2 & 25.8 & 21.0 & 26.2 & 7.2 \\
\hline \multirow[t]{2}{*}{ Domestic expenditures $1 / 2 /$} & 11.5 & 26.8 & 17.5 & 18.6 & 28.2 & 24.4 & 23.4 & 5.4 \\
\hline & \multicolumn{8}{|c|}{ (In percent of GDP) } \\
\hline \multicolumn{9}{|l|}{ Investment and savings } \\
\hline Gross national savings 3/ & 15.5 & 18.1 & 18.6 & 19.5 & 19.6 & 18.5 & 18.1 & 18.6 \\
\hline Gross foreign savings 4 / & 6.5 & 7.5 & 4.6 & 4.8 & 4.1 & 4.3 & 3.9 & 3.9 \\
\hline Gross domestic investment & 22.0 & 25.6 & 23.3 & 24.3 & 23.7 & 22.8 & 22.0 & 22.5 \\
\hline Of which: public investment & 11.8 & 11.4 & 9.2 & 8.8 & 8.3 & 7.1 & 9.5 & 10.1 \\
\hline \multicolumn{9}{|l|}{ Central government budget } \\
\hline Revenue and grants & 26.2 & 24.9 & 24.1 & 23.9 & 24.8 & 24.4 & 25.3 & 25.1 \\
\hline Revenue (excluding grants) & 17.9 & 17.9 & 18.7 & 18.4 & 18.3 & 18.4 & 18.4 & 18.4 \\
\hline Expenditures (excluding interest) 1/ & 27.2 & 27.0 & 23.1 & 23.3 & 23.6 & 23.5 & 26.7 & 26.1 \\
\hline Interest due & 4.1 & 3.9 & 4.2 & 3.5 & 3.3 & 3.4 & 2.8 & 2.8 \\
\hline Domestic expenditures $1 / 2 /$ & 20.7 & 20.9 & 19.5 & 19.6 & 20.2 & 20.2 & 21.7 & 20.6 \\
\hline Overall balance, cash basis & -6.3 & -6.6 & -3.4 & -1.7 & -2.2 & -2.5 & -2.6 & -2.1 \\
\hline Domestic financing & 2.1 & 5.1 & 2.2 & 0.8 & 1.6 & 1.6 & 1.2 & 0.6 \\
\hline \multicolumn{9}{|l|}{ External sector } \\
\hline Current account balance, excluding grants & -17.3 & -16.2 & -11.4 & -11.9 & -11.6 & -11.8 & -12.0 & -11.7 \\
\hline Current account balance, including grants and debt relief $4 /$ & -6.5 & -7.5 & -4.6 & -4.8 & -4.0 & -4.3 & -3.9 & -3.9 \\
\hline & & & percent & exports & ods and & ices) & & \\
\hline External debt & & & & & & & & \\
\hline External official debt service & 11.4 & 15.4 & 23.4 & 20.0 & 6.8 & 7.6 & 4.1 & 5.5 \\
\hline External program assistance 5/ & 13.1 & 4.5 & 4.3 & 3.6 & 6.1 & 6.7 & 4.9 & 3.9 \\
\hline & & (In & ons of $\mathrm{C}$ & dollars, & ss otherv & indicate & & \\
\hline Current account balance, including grants and debt relief 4 / & -246 & -323 & -238 & -260 & -226 & -268 & -252 & -272 \\
\hline Overall balance of payments & -383 & -321 & -283 & -285 & -283 & -347 & -368 & -317 \\
\hline Gross official reserves (end of period) & 283 & 194 & 173 & 222 & 246 & 246 & 310 & 353 \\
\hline In months of imports of goods and services & 2.2 & 1.3 & 1.0 & 1.2 & 1.3 & 1.3 & 1.5 & 1.7 \\
\hline
\end{tabular}

Sources: Zambian authorities; and IMF staff estimates and projections.

1/ Includes contingency reserve and payments of domestic arrears.

2/ Excludes external interest payments and foreign-financed project expenditure.

3/ After enhanced HIPC Initiative debt relief.

4/ Includes program and budget grants and debt relief on interest payments.

5/ For 2005-07, figures are preliminary projections and include only identified commitments. 
Table 2. Zambia: Central Government Overall Operations, 2003-07 (In billions of kwacha)

\begin{tabular}{|c|c|c|c|c|c|c|c|c|c|c|c|}
\hline & \multirow{2}{*}{$\begin{array}{c}2003 \\
\text { Act. }\end{array}$} & \multicolumn{2}{|c|}{2004} & \multirow{2}{*}{$\begin{array}{r}2005 \\
\text { Proj. } \\
\text { Staff Rpt. } 04 \\
\end{array}$} & \multirow{2}{*}{$\begin{array}{l}2005 \\
\text { Prog. }\end{array}$} & \multicolumn{4}{|c|}{2005} & \multirow{2}{*}{$\begin{array}{l}2006 \\
\text { Proj. }\end{array}$} & \multirow{2}{*}{$\begin{array}{l}2007 \\
\text { Proj. }\end{array}$} \\
\hline & & Prog. & Prel. & & & $\begin{array}{r}\text { Prog. } \\
\text { Q1 } \\
\end{array}$ & $\begin{array}{r}\text { Prog. } \\
\text { Q2 } \\
\end{array}$ & $\begin{array}{r}\text { Prog. } \\
\text { Q3 } \\
\end{array}$ & $\begin{array}{r}\text { Prog. } \\
\text { Q4 } \\
\end{array}$ & & \\
\hline Revenue and grants & 5,104 & 6,172 & 6,173 & 7,730 & 7,608 & 1,813 & 1,860 & 1,848 & 2,087 & 9,057 & 9,997 \\
\hline Revenue & 3,680 & 4,784 & 4,740 & 5,696 & 5,739 & 1,306 & 1,400 & 1,482 & 1,551 & 6,600 & 7,341 \\
\hline Tax revenue & 3,548 & 4,547 & 4,546 & 5,479 & 5,523 & 1,246 & 1,360 & 1,451 & 1,466 & 6,347 & 7,065 \\
\hline Income taxes & 1,622 & 2,049 & 2,032 & 2,457 & 2,436 & 555 & 588 & 656 & 636 & 2,795 & 3,112 \\
\hline Excise taxes & 482 & 615 & 607 & 717 & 720 & 163 & 167 & 196 & 194 & 834 & 927 \\
\hline Value-added tax (VAT) & 1,034 & 1,373 & 1,362 & 1,620 & 1,588 & 372 & 402 & 385 & 429 & 1,859 & 2,069 \\
\hline Domestic VAT & 393 & 608 & 453 & 641 & 609 & 147 & 156 & 132 & 175 & 736 & 819 \\
\hline Import VAT & 642 & 766 & 909 & 979 & 979 & 226 & 247 & 253 & 254 & 1,123 & 1,250 \\
\hline Customs duty & 409 & 509 & 544 & 685 & 778 & 156 & 202 & 213 & 207 & 859 & 957 \\
\hline Nontax revenue 1/ & 132 & 239 & 194 & 217 & 217 & 60 & 40 & 32 & 84 & 253 & 277 \\
\hline Grants & 1,424 & 1,388 & 1,433 & 2,034 & 1,869 & 507 & 460 & 366 & 536 & 2,457 & 2,656 \\
\hline Program & 229 & 163 & 258 & 481 & 446 & 151 & 44 & 70 & 180 & 361 & 252 \\
\hline Project 2/ & 1,195 & 1,225 & 1,175 & 1,553 & 1,423 & 356 & 416 & 296 & 356 & 2,095 & 2,404 \\
\hline Expenditures & 6,337 & 6,981 & 6,919 & 8,414 & 8,373 & 1,827 & 2,040 & 2,109 & 2,397 & 10,563 & 11,328 \\
\hline Current expenditures & 4,002 & 4,640 & 4,654 & 5,824 & 6,170 & 1,306 & 1,491 & 1,647 & 1,725 & 7,151 & 7,314 \\
\hline Wages and salaries & 1,728 & 2,018 & 2,012 & 2,457 & 2,531 & 593 & 642 & 640 & 655 & 2,894 & 3,148 \\
\hline Public service retrenchment & 10 & 33 & 20 & 66 & 66 & 13 & 39 & 14 & 0 & 75 & 80 \\
\hline Recurrent departmental charges (RDCs) 3/ & 648 & 686 & 835 & 1,126 & 1,513 & 282 & 301 & 429 & 501 & 1,849 & 1,581 \\
\hline Of which: arrears clearance & 52 & 77 & 84 & 141 & 141 & 25 & 15 & 41 & 59 & 188 & 188 \\
\hline Of which: elections \& constitutional review & 0 & 26 & 19 & 186 & 146 & 30 & 47 & 50 & 19 & 714 & 306 \\
\hline Transfers and pensions & 361 & 419 & 446 & 599 & 760 & 182 & 122 & 194 & 262 & 680 & 718 \\
\hline Of which: Settlement of pension arrears & 5 & 30 & 27 & 142 & 159 & 43 & 28 & 14 & 74 & 154 & 166 \\
\hline Domestic interest & 563 & 855 & 746 & 850 & 850 & 187 & 237 & 209 & 217 & 794 & 738 \\
\hline External interest 4/ & 229 & 213 & 152 & 193 & 200 & 37 & 64 & 44 & 56 & 199 & 194 \\
\hline Other current expenditures & 456 & 405 & 430 & 517 & 243 & 12 & 85 & 114 & 32 & 645 & 837 \\
\hline Of which: financial restructuring & 209 & 120 & 108 & 100 & 100 & 5 & 35 & 47 & 13 & 150 & 180 \\
\hline Contingency & 6 & 11 & 13 & 15 & 8 & 0 & 1 & 4 & 3 & 15 & 18 \\
\hline Capital expenditure & 2,335 & 2,341 & 2,265 & 2,591 & 2,203 & 521 & 549 & 462 & 671 & 3,412 & 4,015 \\
\hline Domestically financed & 507 & 601 & 585 & 661 & 521 & 103 & 70 & 102 & 246 & 824 & 1,076 \\
\hline Foreign financed & 1,828 & 1,740 & 1,681 & 1,930 & 1,682 & 418 & 480 & 359 & 425 & 2,588 & 2,938 \\
\hline Change in balances and statistical discrepancy 5 / & -116 & -70 & 304 & 0 & 0 & 0 & 0 & 0 & 0 & 0 & 0 \\
\hline Additional grants/measures 6/ & $\cdots$ & $\cdots$ & $\cdots$ & $\cdots$ & $\cdots$ & $\ldots$ & $\ldots$ & $\cdots$ & $\ldots$ & 579 & 511 \\
\hline Overall balance (cash basis) & $-1,349$ & -879 & -442 & -685 & -765 & -14 & -180 & -261 & -310 & -927 & -821 \\
\hline Overall balance excluding grants & $-2,774$ & $-2,267$ & $-1,875$ & $-2,719$ & $-2,634$ & -521 & -640 & -626 & -846 & $-3,384$ & $-3,477$ \\
\hline Domestic balance & -716 & -282 & -22 & -596 & -564 & -20 & -50 & -176 & -318 & -596 & -344 \\
\hline Domestic primary balance & -153 & 573 & 724 & 254 & 286 & 120 & 140 & -14 & -148 & 198 & 394 \\
\hline Financing & 1,349 & 879 & 442 & 685 & 765 & 14 & 180 & 261 & 310 & 927 & 821 \\
\hline Domestic 7/ & 1,041 & 573 & 212 & 500 & 500 & 60 & 157 & 84 & 199 & 412 & 245 \\
\hline Bank & 979 & 434 & 45 & 343 & 343 & 29 & 86 & 71 & 157 & 272 & 162 \\
\hline Nonbank & 62 & 139 & 167 & 158 & 157 & 31 & 71 & 13 & 42 & 140 & 83 \\
\hline External & 308 & 306 & 230 & 185 & 264 & -45 & 23 & 176 & 110 & 515 & 576 \\
\hline Program loans & 46 & 181 & 96 & 192 & 220 & 0 & 44 & 98 & 78 & 194 & 217 \\
\hline Project loans 2/ & 633 & 548 & 526 & 377 & 446 & 109 & 111 & 110 & 116 & 493 & 534 \\
\hline Amortization 4/ & -371 & -423 & -392 & -384 & -402 & -155 & -131 & -32 & -84 & -172 & -176 \\
\hline \multicolumn{12}{|l|}{ Memorandum items: } \\
\hline HIPC-related poverty reducing spending & 213 & 534 & 530 & $\cdots$ & $\ldots$ & $\ldots$ & $\ldots$ & $\cdots$ & $\cdots$ & $\cdots$ & $\cdots$ \\
\hline Poverty-reducing spending (wider definition) $8 /$ & $\cdots$ & $\ldots$ & $\cdots$ & $\cdots$ & 3,182 & $\ldots$ & $\cdots$ & $\ldots$ & $\ldots$ & $\cdots$ & ... \\
\hline of which: domestically financed & $\ldots$ & $\ldots$ & $\ldots$ & $\ldots$ & 1,538 & $\ldots$ & $\ldots$ & $\ldots$ & $\ldots$ & $\ldots$ & \\
\hline External budget support & 275 & 344 & 354 & 673 & 666 & 151 & 88 & 168 & 259 & 555 & 469 \\
\hline External debt service & 600 & 636 & 544 & 577 & 603 & 191 & 196 & 76 & 140 & 371 & 370 \\
\hline Stock of domestic arrears (end period) $9 /$ & 747 & 670 & 675 & 529 & 534 & 650 & 635 & 594 & 534 & 346 & 229 \\
\hline Stock of domestic debt (end period) $10 /$ & 4,481 & 5,054 & 4,693 & 5,532 & 5,194 & 4,753 & 4,910 & 4,994 & 5,194 & 5,606 & 5,851 \\
\hline GDP (annual) & 20,520 & 25,578 & 25,814 & 31,172 & 31,212 & 31,212 & 31,212 & 31,212 & 31,212 & 35,812 & 39,865 \\
\hline
\end{tabular}

Sources: Zambian authorities; and IMF staff estimates and projections.

1/ In 2004, nontax revenues include K 62 billion of additional proceeds due to the reconciliation of government accounts.

$2 /$ In 2005, project grants and loans are also funding current expenditures.

3/ Include purchases of goods and services, some allowances, and training expenses.

4/ The Paris Club has agreed to provide debt relief on Cologne flow terms up to end-June 2005. Once the completion point under the HIPC Initiative is reached, the Paris club is expected to provide debt relief on Cologne stock terms.

5/ Payments to Roan Antelope Mining Company of Zambia employees for retrenchment (K 70 billion) released in 2003:Q4 were cashed in 2004:Q1. Authorized expenditure of about 0.3 percent of GDP which could not be spent in 2004 due to late funding is expected to be executed in 2005 .

6/ Possible measures will not affect poverty-reducing programs.

7/ Until 2003 at face value. From 2004 at cost value. Excludes bonds issued in 2001 in favor of the Bank of Zambia (K 1,646 billion) and in 2002 in favor of the Zambia National Commercial Bank (K 248 billions).

8/ From 2005, fiscal accounts will identify poverty-reducing spending according to a wider definition consistent with the PRSP (MEFP, para. 14).

9/ Excludes the stock of statutory pension arrears, which was K 322 billion at end-2003. For 2004, preliminary data are estimates.

10/ At cost value. Includes bonds issued in 2001 in favor of BoZ (K 1,646 billion) and in 2002 in favor of the Zambia National Commercial Bank (K 248 billion). 
Table 3. Zambia: Central Government Overall Operations, 2003-07 (In percent of GDP)

\begin{tabular}{|c|c|c|c|c|c|c|c|c|c|c|c|}
\hline & \multirow{2}{*}{$\begin{array}{c}2003 \\
\text { Act. }\end{array}$} & \multicolumn{2}{|c|}{2004} & \multirow{2}{*}{$\begin{array}{r}2005 \\
\text { Proj. } \\
\text { Staff Rpt. } 04\end{array}$} & \multirow{2}{*}{$\begin{array}{c}2005 \\
\text { Prog. }\end{array}$} & \multicolumn{4}{|c|}{2005} & \multirow{2}{*}{$\begin{array}{l}2006 \\
\text { Proj. }\end{array}$} & \multirow{2}{*}{$\begin{array}{l}2007 \\
\text { Proj. }\end{array}$} \\
\hline & & Prog. & Prel. & & & $\begin{array}{r}\text { Prog. } \\
\text { Q1 }\end{array}$ & $\begin{array}{r}\text { Prog. } \\
\text { Q2 }\end{array}$ & $\begin{array}{r}\text { Prog. } \\
\text { Q3 }\end{array}$ & $\begin{array}{r}\text { Prog. } \\
\text { Q4 }\end{array}$ & & \\
\hline Revenue and grants & 24.9 & 24.1 & 23.9 & 24.8 & 24.4 & 5.8 & 6.0 & 5.9 & 6.7 & 25.3 & 25.1 \\
\hline Revenue & 17.9 & 18.7 & 18.4 & 18.3 & 18.4 & 4.2 & 4.5 & 4.7 & 5.0 & 18.4 & 18.4 \\
\hline Tax revenue & 17.3 & 17.8 & 17.6 & 17.6 & 17.7 & 4.0 & 4.4 & 4.6 & 4.7 & 17.7 & 17.7 \\
\hline Income taxes & 7.9 & 8.0 & 7.9 & 7.9 & 7.8 & 1.8 & 1.9 & 2.1 & 2.0 & 7.8 & 7.8 \\
\hline Excise taxes & 2.3 & 2.4 & 2.4 & 2.3 & 2.3 & 0.5 & 0.5 & 0.6 & 0.6 & 2.3 & 2.3 \\
\hline Value-added tax (VAT) & 5.0 & 5.4 & 5.3 & 5.2 & 5.1 & 1.2 & 1.3 & 1.2 & 1.4 & 5.2 & 5.2 \\
\hline Domestic VAT & 1.9 & 2.4 & 1.8 & 2.1 & 2.0 & 0.5 & 0.5 & 0.4 & 0.6 & 2.1 & 2.1 \\
\hline Import VAT & 3.1 & 3.0 & 3.5 & 3.1 & 3.1 & 0.7 & 0.8 & 0.8 & 0.8 & 3.1 & 3.1 \\
\hline Customs duty & 2.0 & 2.0 & 2.1 & 2.2 & 2.5 & 0.5 & 0.6 & 0.7 & 0.7 & 2.4 & 2.4 \\
\hline Nontax revenue $1 /$ & 0.6 & 0.9 & 0.8 & 0.7 & 0.7 & 0.2 & 0.1 & 0.1 & 0.3 & 0.7 & 0.7 \\
\hline Grants & 6.9 & 5.4 & 5.6 & 6.5 & 6.0 & 1.6 & 1.5 & 1.2 & 1.7 & 6.9 & 6.7 \\
\hline Program & 1.1 & 0.6 & 1.0 & 1.5 & 1.4 & 0.5 & 0.1 & 0.2 & 0.6 & 1.0 & 0.6 \\
\hline Project 2/ & 5.8 & 4.8 & 4.6 & 5.0 & 4.6 & 1.1 & 1.3 & 0.9 & 1.1 & 5.9 & 6.0 \\
\hline Expenditures & 30.9 & 27.3 & 26.8 & 27.0 & 26.8 & 5.9 & 6.5 & 6.8 & 7.7 & 29.5 & 28.4 \\
\hline Current expenditures & 19.5 & 18.1 & 18.0 & 18.7 & 19.8 & 4.2 & 4.8 & 5.3 & 5.5 & 20.0 & 18.3 \\
\hline Wages and salaries & 8.4 & 7.9 & 7.8 & 7.9 & 8.1 & 1.9 & 2.1 & 2.1 & 2.1 & 8.1 & 7.9 \\
\hline Public service retrenchment & 0.0 & 0.1 & 0.1 & 0.2 & 0.2 & 0.0 & 0.1 & 0.0 & 0.0 & 0.2 & 0.2 \\
\hline Recurrent departmental charges (RDCs) 3/ & 3.2 & 2.7 & 3.2 & 3.6 & 4.8 & 0.9 & 1.0 & 1.4 & 1.6 & 5.2 & 4.0 \\
\hline Of which: arrears clearance & 0.3 & 0.3 & 0.3 & 0.5 & 0.5 & 0.1 & 0.0 & 0.1 & 0.2 & 0.5 & 0.5 \\
\hline Of which: Elections \& constitutional review & 0.0 & 0.1 & 0.1 & 0.6 & 0.5 & 0.1 & 0.1 & 0.2 & 0.1 & 2.0 & 0.8 \\
\hline Transfers and pensions & 1.8 & 1.6 & 1.7 & 1.9 & 2.4 & 0.6 & 0.4 & 0.6 & 0.8 & 1.9 & 1.8 \\
\hline Of which: Settlement of pension arrears & 0.0 & 0.1 & 0.1 & 0.5 & 0.5 & 0.1 & 0.1 & 0.0 & 0.2 & 0.4 & 0.4 \\
\hline Domestic interest & 2.7 & 3.3 & 2.9 & 2.7 & 2.7 & 0.6 & 0.8 & 0.7 & 0.7 & 2.2 & 1.9 \\
\hline External interest 4/ & 1.1 & 0.8 & 0.6 & 0.6 & 0.6 & 0.1 & 0.2 & 0.1 & 0.2 & 0.6 & 0.5 \\
\hline Other current expenditures & 2.2 & 1.6 & 1.7 & 1.7 & 0.8 & 0.0 & 0.3 & 0.4 & 0.1 & 1.8 & 2.1 \\
\hline Of which: financial restructuring & 1.0 & 0.5 & 0.4 & 0.3 & 0.3 & 0.0 & 0.1 & 0.2 & 0.0 & 0.4 & 0.5 \\
\hline Contingency & 0.0 & 0.0 & 0.1 & 0.0 & 0.0 & 0.0 & 0.0 & 0.0 & 0.0 & 0.0 & 0.0 \\
\hline Capital expenditure & 11.4 & 9.2 & 8.8 & 8.3 & 7.1 & 1.7 & 1.8 & 1.5 & 2.1 & 9.5 & 10.1 \\
\hline Domestically financed & 2.5 & 2.4 & 2.3 & 2.1 & 1.7 & 0.3 & 0.2 & 0.3 & 0.8 & 2.3 & 2.7 \\
\hline Foreign financed & 8.9 & 6.8 & 6.5 & 6.2 & 5.4 & 1.3 & 1.5 & 1.2 & 1.4 & 7.2 & 7.4 \\
\hline Change in balances and statistical discrepancy $5 /$ & -0.6 & -0.3 & 1.2 & 0.0 & 0.0 & 0.0 & 0.0 & 0.0 & 0.0 & 0.0 & 0.0 \\
\hline Additional grants/measures $6 /$ & $\ldots$ & $\ldots$ & $\ldots$ & $\ldots$ & $\ldots$ & $\ldots$ & $\ldots$ & $\ldots$ & $\ldots$ & 1.6 & 1.3 \\
\hline Overall balance (cash basis) & -6.6 & -3.4 & -1.7 & -2.2 & -2.5 & 0.0 & -0.6 & -0.8 & -1.0 & -2.6 & -2.1 \\
\hline Overall balance excluding grants & -13.5 & -8.9 & -7.3 & -8.7 & -8.4 & -1.7 & -2.1 & -2.0 & -2.7 & -9.4 & -8.7 \\
\hline Domestic balance & -3.5 & -1.1 & -0.1 & -1.9 & -1.8 & -0.1 & -0.2 & -0.6 & -1.0 & -1.7 & -0.9 \\
\hline Domestic primary balance & -0.7 & 2.2 & 2.8 & 0.8 & 0.9 & 0.4 & 0.4 & 0.0 & -0.5 & 0.6 & 1.0 \\
\hline Financing & 6.6 & 3.4 & 1.7 & 2.2 & 2.5 & 0.0 & 0.6 & 0.8 & 1.0 & 2.6 & 2.1 \\
\hline Domestic 7/ & 5.1 & 2.2 & 0.8 & 1.6 & 1.6 & 0.2 & 0.5 & 0.3 & 0.6 & 1.2 & 0.6 \\
\hline Bank & 4.8 & 1.7 & 0.2 & 1.1 & 1.1 & 0.1 & 0.3 & 0.2 & 0.5 & 0.8 & 0.4 \\
\hline Nonbank & 0.3 & 0.5 & 0.6 & 0.5 & 0.5 & 0.1 & 0.2 & 0.0 & 0.1 & 0.4 & 0.2 \\
\hline External & 1.5 & 1.2 & 0.9 & 0.6 & 0.8 & -0.1 & 0.1 & 0.6 & 0.4 & 1.4 & 1.4 \\
\hline Program loans & 0.2 & 0.7 & 0.4 & 0.6 & 0.7 & 0.0 & 0.1 & 0.3 & 0.3 & 0.5 & 0.5 \\
\hline Project loans 2/ & 3.1 & 2.1 & 2.0 & 1.2 & 1.4 & 0.3 & 0.4 & 0.4 & 0.4 & 1.4 & 1.3 \\
\hline Amortization 4/ & -1.8 & -1.7 & -1.5 & -1.2 & -1.3 & -0.5 & -0.4 & -0.1 & -0.3 & -0.5 & -0.4 \\
\hline \multicolumn{12}{|l|}{ Memorandum items: } \\
\hline HIPC-related poverty reducing spending & 1.0 & 2.1 & 2.1 & $\ldots$ & $\ldots$ & $\ldots$ & $\ldots$ & $\ldots$ & $\ldots$ & $\ldots$ & $\ldots$ \\
\hline Poverty-reducing spending (wider definition) 8/ & $\ldots$ & $\ldots$ & $\ldots$ & $\ldots$ & 10.0 & $\ldots$ & $\ldots$ & $\ldots$ & $\ldots$ & $\ldots$ & $\ldots$ \\
\hline of which: domestically financed & $\ldots$ & $\ldots$ & $\ldots$ & $\ldots$ & 4.9 & $\ldots$ & $\ldots$ & $\ldots$ & $\ldots$ & $\ldots$ & $\ldots$ \\
\hline External budget support & 1.3 & 1.3 & 1.4 & 2.2 & 2.1 & 0.5 & 0.3 & 0.5 & 0.8 & 1.6 & 1.2 \\
\hline External debt service & 2.9 & 2.5 & 2.1 & 1.8 & 1.9 & 0.6 & 0.6 & 0.2 & 0.4 & 1.0 & 0.9 \\
\hline Stock of domestic arrears (end period) 9/ & 3.6 & 2.6 & 2.6 & 1.7 & 1.7 & 2.1 & 2.0 & 1.9 & 1.7 & 1.0 & 0.6 \\
\hline Stock of domestic debt (end period) $10 /$ & 21.8 & 19.8 & 18.2 & 17.7 & 16.6 & 15.2 & 15.7 & 16.0 & 16.6 & 15.7 & 14.7 \\
\hline GDP (annual) & 20,520 & 25,578 & 25,814 & 31,172 & 31,212 & 31,212 & 31,212 & 31,212 & 31,212 & 35,812 & 39,865 \\
\hline
\end{tabular}

1/ In 2004, nontax revenues include K 62 billion of additional proceeds due to the reconciliation of government accounts.

2/ In 2005, project grants and loans are also funding current expenditures.

3/ Include purchases of goods and services, some allowances, and training expenses.

4/ The Paris Club has agreed to provide debt relief on Cologne flow terms up to end-June 2005. Once the completion point under the HIPC Initiative is reached, the Paris club is expected to provide debt relief on Cologne stock terms.

5/ Payments to Roan Antelope Mining Company of Zambia employees for retrenchment (K 70 billion) released in 2003:Q4 were cashed in 2004:Q1.

Authorized expenditure of about 0.3 percent of GDP which could not be spent in 2004 due to late funding is expected to be executed in 2005 .

6/ Possible measures will not affect poverty-reducing programs.

7/ Until 2003 at face value. From 2004 at cost value. Excludes bonds issued in 2001 in favor of the Bank of Zambia (K 1,646 billion) and in 2002 in favor of the Zambia National Commercial Bank (K 248 billions).

8/ From 2005, fiscal accounts will identify poverty-reducing spending according to a wider definition consistent with the PRSP (MEFP, para. 14).

9/ Excludes the stock of statutory pension arrears, which was K 322 billion at end-2003. For 2004, preliminary data are estimates.

10/ At cost value. Includes bonds issued in 2001 in favor of BoZ (K 1,646 billion) and in 2002 in favor of the Zambia National Commercial Bank (K 248 billion). 
Table 4. Zambia: Monetary Survey, 2002-05

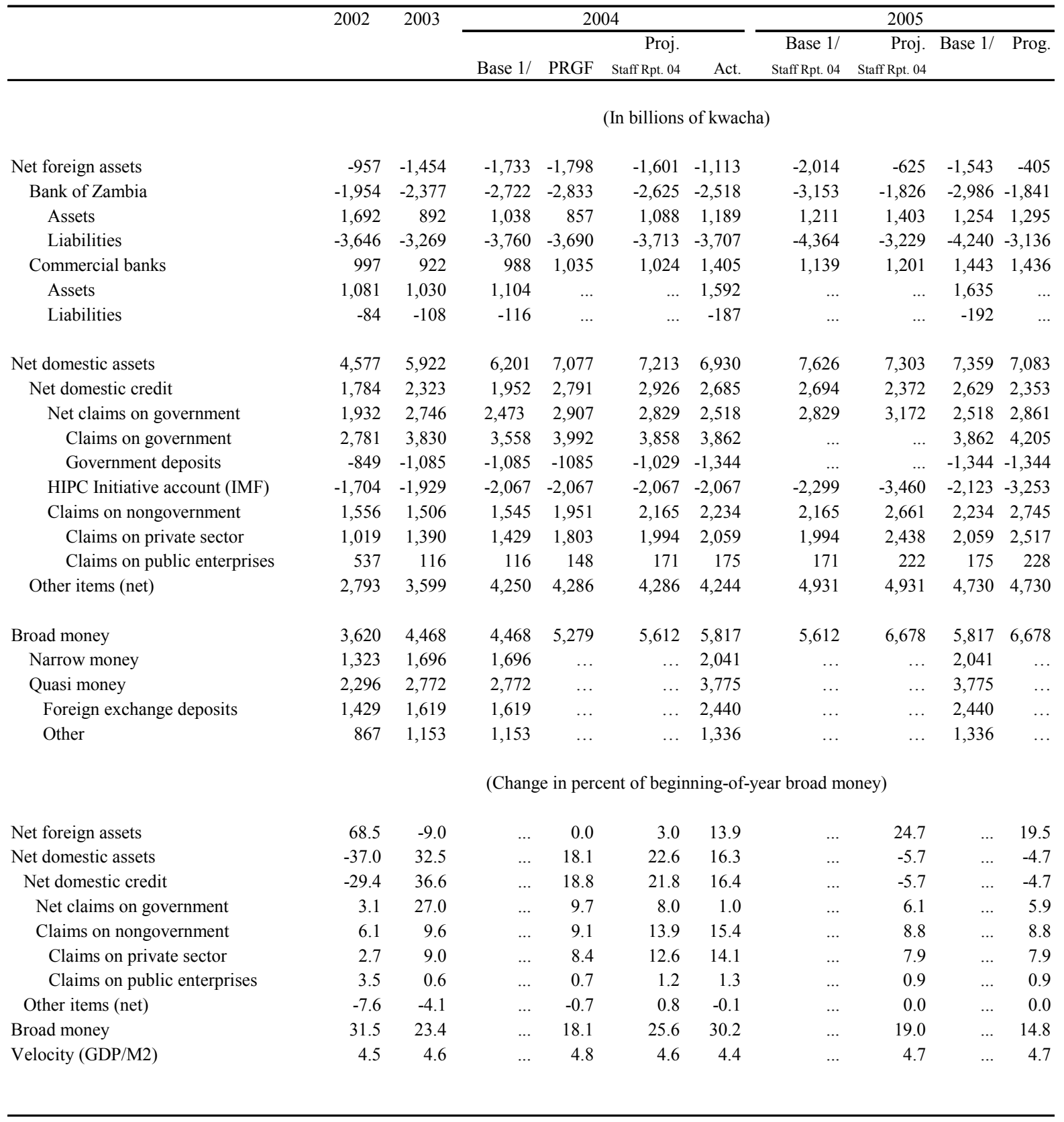

Sources: Zambian authorities; and IMF staff estimates and projections.

1 / The base for the year $t$ is obtained recalculating the figures for end-year $t-1$ on the basis of the program exchange rates assumed for the year $t$. Beginning in 2004, government securities are recorded at cost rather than at face value. 
Table 4. Zambia: Monetary Survey, 2002-05 (concluded)

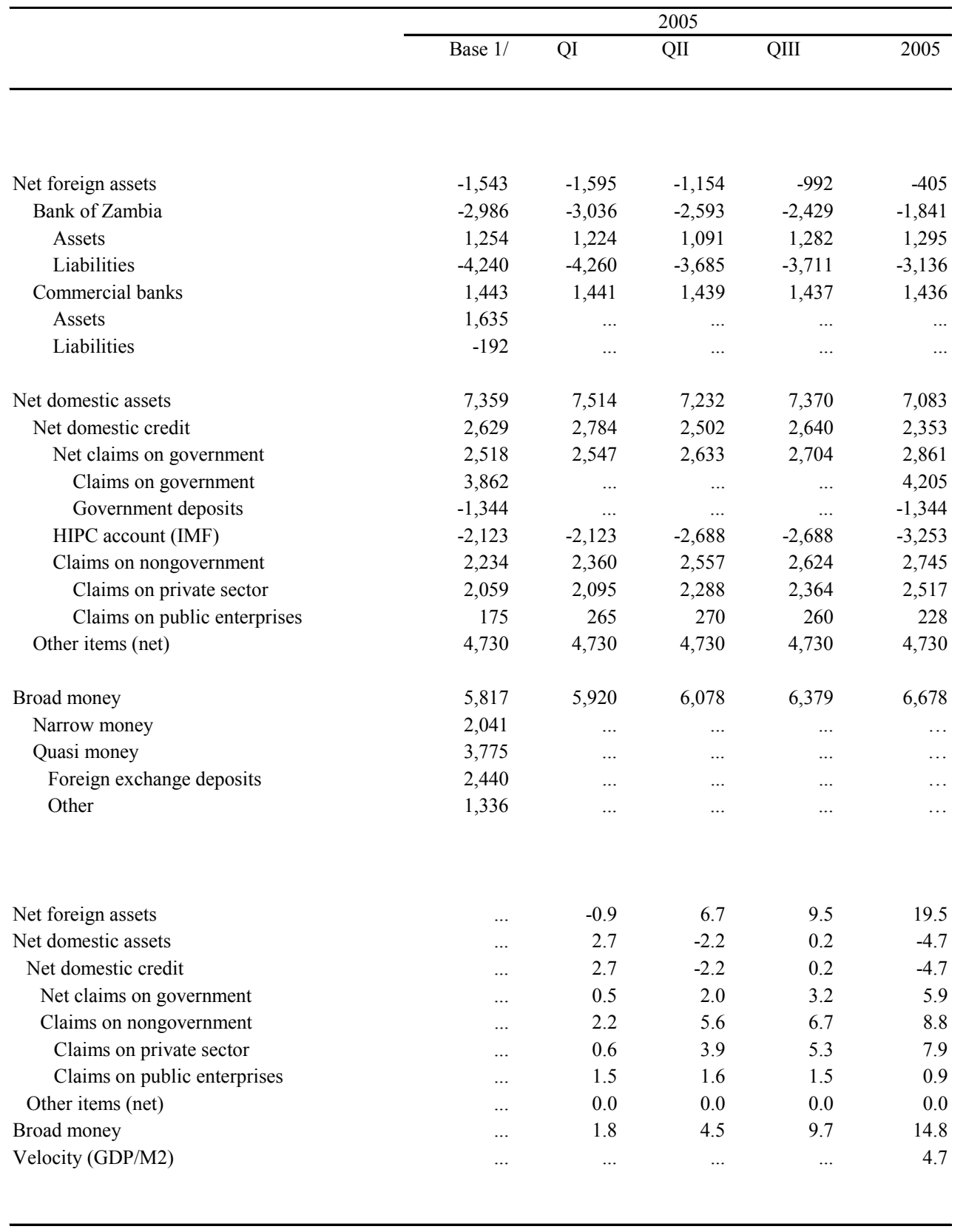

Sources: Zambian authorities; and IMF staff estimates and projections.

1/ The base for the year $t$ is obtained recalculating the figures for end year $t-1$ on the basis of the program exchange rates assumed for the year t. Beginning in 2004, government securities are recorded at cost rather than at face value. 
Table 5. Zambia: Assets and Liabilities of the Bank of Zambia, 2002-05 (In billions of kwacha, unless otherwise indicated)

\begin{tabular}{|c|c|c|c|c|c|c|c|c|c|c|}
\hline & \multirow[t]{3}{*}{2002} & \multirow[t]{3}{*}{2003} & \multicolumn{4}{|c|}{2004} & \multicolumn{4}{|c|}{2005} \\
\hline & & & \multirow[b]{2}{*}{ Base 1/ } & \multirow[b]{2}{*}{ PRGF } & \multicolumn{2}{|l|}{ Proj. } & \multirow{2}{*}{$\begin{array}{r}\text { Base 1/ } \\
\text { Staff Rpt. 04 } \\
\end{array}$} & \multirow{2}{*}{$\begin{array}{r}\text { Proj. } \\
\text { Staff Rpt. } 04 \\
\end{array}$} & \multirow[t]{2}{*}{ Base 1/ } & \multirow[t]{2}{*}{ Prog. } \\
\hline & & & & & Staff Rpt. 04 & Act. & & & & \\
\hline Net foreign assets & $-1,954$ & $-2,377$ & $-2,722$ & $-2,833$ & $-2,625$ & $-2,518$ & $-3,153$ & $-1,826$ & $-2,986$ & $-1,841$ \\
\hline Assets & 1,692 & 892 & 1,038 & 857 & 1,088 & 1,189 & 1,211 & 1,403 & 1,254 & 1,295 \\
\hline Liabilities & $-3,646$ & $-3,269$ & $-3,760$ & $-3,690$ & $-3,713$ & $-3,707$ & $-4,364$ & $-3,229$ & $-4,240$ & $-3,136$ \\
\hline Of which: IMF (net) & $-3,603$ & $-3,242$ & $-3,731$ & $-3,684$ & $-3,707$ & $-3,701$ & $-4,357$ & $-3,229$ & $-4,234$ & $-3,136$ \\
\hline Net domestic assets & 3,158 & 3,796 & 4,141 & 4,334 & 4,220 & 4,237 & 4,748 & 3,724 & 4,713 & 3,749 \\
\hline Net domestic credit & -189 & -476 & -615 & -518 & -376 & -516 & -776 & $-1,799$ & -572 & $-1,536$ \\
\hline Net claims on government & 1,128 & 1,090 & 1,090 & 1,120 & 1,236 & 1,241 & 1,068 & 1,119 & 1,241 & 1,361 \\
\hline Claims on government & 1,865 & 1,973 & $\ldots$ & $\ldots$ & $\ldots$ & $\ldots$ & $\ldots$ & $\ldots$ & $\ldots$ & $\ldots$ \\
\hline Government deposits & -737 & -883 & $\ldots$ & $\ldots$ & $\ldots$ & $\ldots$ & $\ldots$ & $\ldots$ & $\ldots$ & $\ldots$ \\
\hline HIPC Initiative account (IMF) & $-1,704$ & $-1,929$ & $-2,067$ & $-2,067$ & $-2,067$ & $-2,067$ & $-2,299$ & $-3,460$ & $-2,123$ & $-3,253$ \\
\hline Claims on nongovernment & 387 & 363 & 363 & 429 & 456 & 310 & 456 & 542 & 310 & 356 \\
\hline Of which: claims on banks & 112 & 262 & $\ldots$ & $\ldots$ & $\ldots$ & $\ldots$ & $\ldots$ & $\ldots$ & $\ldots$ & $\ldots$ \\
\hline Other items (net) & 3,347 & 4,272 & 4,755 & 4,734 & 4,764 & 4,753 & 5,524 & 5,524 & 5,285 & 5,285 \\
\hline Open market operations & $\ldots$ & 0 & 0 & 118 & -168 & 0 & 0 & 0 & 0 & 0 \\
\hline Reserve money & 1,205 & 1,419 & 1,419 & 1,501 & 1,595 & 1,719 & 1,595 & 1,898 & 1,727 & 1,908 \\
\hline Currency in circulation & 479 & 670 & 670 & $\ldots$ & $\ldots$ & 816 & $\ldots$ & $\ldots$ & 816 & $\ldots$ \\
\hline Required reserves (kwacha deposits) & 264 & 286 & 286 & $\ldots$ & $\ldots$ & 352 & $\ldots$ & $\ldots$ & 352 & $\ldots$ \\
\hline Required reserves (foreign exchange deposits) & 234 & 224 & 224 & $\cdots$ & $\ldots$ & 295 & $\ldots$ & $\cdots$ & 303 & $\ldots$ \\
\hline Dollar denominated & 86 & 224 & 224 & $\ldots$ & $\ldots$ & 295 & $\ldots$ & $\ldots$ & 303 & $\ldots$ \\
\hline Kwacha denominated & 148 & 0 & 0 & $\ldots$ & $\ldots$ & 0 & $\ldots$ & $\ldots$ & 0 & $\ldots$ \\
\hline Current accounts & 222 & 233 & 233 & $\ldots$ & $\ldots$ & 249 & $\ldots$ & $\ldots$ & 249 & $\ldots$ \\
\hline Nongovernment deposits & 6 & 6 & 6 & $\cdots$ & $\cdots$ & 7 & $\cdots$ & $\cdots$ & 7 & ... \\
\hline \multicolumn{11}{|l|}{ Memorandum items: } \\
\hline Multiplier (broad money/reserve money) & 3.0 & 3.1 & $\ldots$ & 3.5 & 3.5 & 3.4 & $\ldots$ & 3.5 & $\ldots$ & 3.5 \\
\hline Reserve money (percent change from end of previous year) & $\ldots$ & 17.8 & $\ldots$ & 5.7 & 12.4 & 21.1 & $\cdots$ & 19.0 & $\cdots$ & 10.5 \\
\hline
\end{tabular}

Sources: Zambian authorities; and IMF staff estimates and projections.

1 / The base for the year $t$ is obtained by recalculating the figures for end year $t-1$ on the basis of the program exchange rates assumed for the year $t$. Beginning in 2004, government securities are recorded at cost rather than at face value. Base for 2004 revised in October 2004. 
Table 5. Zambia: Assets and Liabilities of the Bank of Zambia, 2002-05 (concluded)

(In billions of kwacha, unless otherwise indicated)

\begin{tabular}{|c|c|c|c|c|c|}
\hline & \multicolumn{5}{|c|}{2005} \\
\hline & Base 1/ & QI & QII & QIII & 2005 \\
\hline Net foreign assets & $-2,986$ & $-3,036$ & $-2,593$ & $-2,429$ & $-1,841$ \\
\hline Assets & 1,254 & 1,224 & 1,091 & 1,282 & 1,295 \\
\hline Liabilities & $-4,240$ & $-4,260$ & $-3,685$ & $-3,711$ & $-3,136$ \\
\hline Of which: IMF (net) & $-4,234$ & $-4,260$ & $-3,685$ & $-3,711$ & $-3,136$ \\
\hline Net domestic assets & 4,713 & 4,727 & 4,330 & 4,252 & 3,749 \\
\hline Net domestic credit & -572 & -558 & -955 & $-1,033$ & $-1,536$ \\
\hline Net claims on government & 1,241 & 1,246 & 1,403 & 1,315 & 1,361 \\
\hline Claims on government & $\ldots$ & $\ldots$ & $\ldots$ & $\ldots$ & $\ldots$ \\
\hline Government deposits & $\ldots$ & $\ldots$ & $\ldots$ & $\ldots$ & $\ldots$ \\
\hline HIPC account (IMF) & $-2,123$ & $-2,123$ & $-2,688$ & $-2,688$ & $-3,253$ \\
\hline Claims on nongovernment & 310 & 320 & 330 & 340 & 356 \\
\hline Of which: claims on banks & $\ldots$ & $\ldots$ & $\ldots$ & $\ldots$ & $\ldots$ \\
\hline Other items (net) & 5,285 & 5,285 & 5,285 & 5,285 & 5,285 \\
\hline Open market operations & 0 & 0 & 0 & 0 & 0 \\
\hline Reserve money & 1,727 & 1,691 & 1,737 & 1,822 & 1,908 \\
\hline Currency in circulation & 816 & $\ldots$ & $\ldots$ & $\ldots$ & $\ldots$ \\
\hline Required reserves (kwacha deposits) & 352 & $\ldots$ & $\ldots$ & $\ldots$ & $\ldots$ \\
\hline Required reserves (foreign exchange deposits) & 303 & $\ldots$ & $\ldots$ & $\ldots$ & $\ldots$ \\
\hline Dollar denominated & 303 & $\ldots$ & $\ldots$ & $\ldots$ & $\ldots$ \\
\hline Kwacha denominated & 0 & $\ldots$ & $\ldots$ & $\ldots$ & $\ldots$ \\
\hline Current accounts & 249 & $\ldots$ & $\ldots$ & $\ldots$ & $\ldots$ \\
\hline Nongovernment deposits & 7 & $\ldots$ & $\ldots$ & $\ldots$ & $\ldots$ \\
\hline \multicolumn{6}{|l|}{ Memorandum items: } \\
\hline Multiplier (broad money/reserve money) & $\ldots$ & 3.5 & 3.5 & 3.5 & 3.5 \\
\hline Reserve money (percent change from end of previous year) & $\ldots$ & -2.1 & 0.6 & 5.5 & 10.5 \\
\hline
\end{tabular}

Sources: Zambian authorities; and IMF staff estimates and projections.

1/ The base for the year $t$ is obtained recalculating the figures for end year $t-1$ on the basis of the program exchange rates assumed for the year $t$. Beginning in 2004, government securities are recorded at cost rather than at face value. Base for 2004 revised in October 2004. 
Table 6: Zambia: Balance of Payments, 2000-07 (In millions of U.S. dollars, unless otherwise indicated)

\begin{tabular}{|c|c|c|c|c|c|c|c|c|c|}
\hline & \multirow{2}{*}{$\begin{array}{r}2000 \\
\text { Est. }\end{array}$} & \multirow{2}{*}{$\begin{array}{r}2001 \\
\text { Est. }\end{array}$} & \multirow{2}{*}{$\begin{array}{c}2002 \\
\text { Est. }\end{array}$} & \multirow{2}{*}{$\begin{array}{r}2003 \\
\text { Est. }\end{array}$} & \multicolumn{2}{|c|}{2004} & \multirow{2}{*}{$\begin{array}{l}2005 \\
\text { Proj. }\end{array}$} & \multirow{2}{*}{$\begin{array}{r}2006 \\
\text { Proj. }\end{array}$} & \multirow{2}{*}{$\begin{array}{r}2007 \\
\text { Proj. } \\
\end{array}$} \\
\hline & & & & & Prog. & Est. & & & \\
\hline Current account balance $1 /$ & -622 & -758 & -652 & -700 & -589 & -642 & -733 & -788 & -819 \\
\hline Trade balance on goods and nonfactor services & -446 & -570 & -504 & -549 & -433 & -363 & -266 & -397 & -375 \\
\hline Merchandise trade balance & -221 & -342 & -259 & -311 & -166 & -109 & 54 & -70 & -41 \\
\hline Exports, f.o.b. & 746 & 884 & 916 & 1,052 & 1,403 & 1,588 & 1,821 & 1883 & 1905 \\
\hline Metals & 497 & 590 & 560 & 669 & 949 & 1,103 & 1,288 & 1307 & 1278 \\
\hline Nonmetal & 249 & 295 & 357 & 383 & 454 & 485 & 533 & 577 & 627 \\
\hline Imports, f.o.b. & -978 & $-1,253$ & $-1,204$ & $-1,393$ & $-1,599$ & $-1,727$ & $-1,799$ & -1987 & -1982 \\
\hline Metal sector & -177 & -310 & -176 & -169 & -156 & -286 & -290 & -334 & -267 \\
\hline Nonmetal & -801 & -943 & $-1,028$ & $-1,224$ & $-1,443$ & $-1,441$ & $-1,509$ & -1653 & -1715 \\
\hline Goods procured by airlines & 11 & 27 & 28 & 29 & 30 & 31 & 32 & 34 & 35 \\
\hline Services, nonfactor (net) & -225 & -228 & -245 & -238 & -267 & -254 & -320 & -327 & -334 \\
\hline Income (net) & -158 & -168 & -155 & -148 & -132 & -304 & -435 & -354 & -405 \\
\hline Of which: official interest payments & -155 & -144 & -137 & -131 & -120 & -121 & -110 & -101 & -95 \\
\hline Current transfers (net) & -18 & -20 & 7 & -3 & -24 & 25 & -31 & -36 & -39 \\
\hline Capital and financial accounts & 202 & 466 & 238 & 380 & 306 & 260 & 386 & 420 & 502 \\
\hline Project grants & 153 & 222 & 236 & 240 & 246 & 246 & 284 & 383 & 420 \\
\hline Financial account & 49 & 245 & 2 & 140 & 60 & 14 & 102 & 37 & 82 \\
\hline Official loan disbursement (net) & -140 & -96 & -122 & -141 & -106 & -221 & -136 & -77 & -80 \\
\hline Disbursement & 93 & 136 & 111 & 101 & 110 & 110 & 89 & 90 & 93 \\
\hline Amortization (-) & -233 & -233 & -234 & -242 & -216 & -331 & -225 & -167 & -174 \\
\hline Change in NFA of commercial banks (- increase) & -89 & 40 & -53 & 48 & -5 & -90 & 17 & -10 & -10 \\
\hline Private capital (net) & 278 & 301 & 178 & 233 & 171 & 324 & 221 & 125 & 172 \\
\hline Foreign direct investment & 122 & 72 & 178 & 172 & 192 & 344 & 250 & 158 & 187 \\
\hline Private borrowing (net) & 156 & 229 & 0 & 61 & -21 & -20 & -29 & -33 & -15 \\
\hline Errors and omissions, short-term capital & 111 & -107 & 31 & -2 & 0 & 97 & 0 & 0 & 0 \\
\hline Overall balance & -309 & -399 & -383 & -321 & -283 & -285 & -347 & -368 & -317 \\
\hline Financing & 309 & 399 & 383 & 321 & 283 & 285 & 347 & 251 & 207 \\
\hline Change in net int. reserves of BoZ (- increase) & -155 & -124 & -225 & -161 & 12 & -44 & -266 & -48 & -66 \\
\hline Gross official reserves of BoZ (- increase) & -68 & 0 & -169 & 89 & 24 & -28 & -24 & -64 & -43 \\
\hline IMF (net) & -80 & -119 & -50 & -244 & -5 & -10 & -235 & 17 & -23 \\
\hline Disbursements & 26 & 94 & 173 & 0 & 236 & 248 & 34 & 32 & 16 \\
\hline Repayments & -106 & -213 & -222 & -244 & -241 & -257 & -269 & -15 & -39 \\
\hline Debt relief & 217 & 436 & 437 & 389 & 276 & 264 & 480 & 197 & 191 \\
\hline Non-HIPC Initiative 2/ & 217 & 170 & 171 & 154 & 120 & 245 & 152 & 70 & 64 \\
\hline HIPC Initiative, including IMF 3/ & $\ldots$ & 266 & 266 & 235 & 156 & 19 & 328 & 127 & 128 \\
\hline Of which: IMF & $\ldots$ & 150 & 153 & 169 & 0 & 2 & 237 & 8 & 4 \\
\hline Of which: Paris Club 4/ & $\ldots$ & 65 & 64 & 16 & 100 & -2 & 38 & 51 & 56 \\
\hline Program support & 185 & 75 & 138 & 55 & 69 & 65 & 133 & 101 & 82 \\
\hline Grants & 32 & 31 & 69 & 45 & 33 & 44 & 89 & 66 & 44 \\
\hline Loans & 154 & 44 & 69 & 10 & 36 & 21 & 44 & 35 & 38 \\
\hline Financing Gap ( $+=$ deficit $)$ & 0 & 0 & 0 & 0 & 0 & 0 & 0 & 117 & 109 \\
\hline \multicolumn{10}{|l|}{ Memorandum items: } \\
\hline Current acc't bal. excl. grants (percent of GDP) & -19.2 & -20.8 & -17.3 & -16.2 & -11.4 & -11.9 & -11.8 & -12.0 & -11.7 \\
\hline Current acc't bal. incl. grants (US\$m) 5/ & -369 & -394 & -246 & -323 & -238 & -260 & -268 & -252 & -272 \\
\hline Current acc't bal. incl. grants (percent of GDP) 5/ & -11.4 & -10.8 & -6.5 & -7.5 & -4.6 & -4.8 & -4.3 & -3.9 & -3.9 \\
\hline Merchandise trade balance (percent of GDP) & -6.8 & -9.4 & -6.9 & -7.2 & -3.2 & -2.0 & 0.9 & -1.1 & -0.6 \\
\hline Terms of trade (percentage change) & -4.2 & -1.7 & -6.7 & 4.2 & 10.7 & 20.3 & -1.0 & -6.8 & -2.9 \\
\hline Copper volume (thousands of metric tons) & 234 & 297 & 330 & 353 & 382 & 384 & 469 & 538 & 563 \\
\hline Copper price (U.S. dollars per pound) & 0.82 & 0.77 & 0.70 & 0.78 & 0.96 & 1.16 & 1.09 & 0.90 & 0.82 \\
\hline Gross official reserves $6 /$ & 114 & 114 & 283 & 194 & 173 & 222 & 246 & 310 & 353 \\
\hline (in months of imports) & 1.0 & 0.9 & 2.2 & 1.3 & 1.0 & 1.2 & 1.3 & 1.5 & 1.7 \\
\hline Official debt service, cash payments & 139 & 142 & 123 & 192 & 376 & 371 & 152 & 86 & 117 \\
\hline (in percent of exports) & 15.9 & 13.5 & 11.4 & 15.4 & 23.4 & 20.0 & 7.6 & 4.1 & 5.5 \\
\hline Official budget debt service, cash payments & 130 & 70 & 50 & 108 & 129 & 114 & 120 & 67 & 73 \\
\hline Net present value of debt, percent of exports & 213 & 208 & 205 & 177 & 181 & 141 & 117 & 104 & 101 \\
\hline
\end{tabular}

Sources: Bank of Zambia (BoZ); and IMF staff estimates and projections.

1/ Excludes grants and debt relief on interest.

2/ Indicates debt relief that would have been available under the traditional mechanism, relative to 1999 Paris Club rescheduling, comparable treatment from non-Paris Club bilaterals.

3/ It is assumed that Zambia will reach the HIPC completion point in the first half of 2005.

Initiative

is reached, the Paris Club is expected to provide debt relief on Cologne stock terms. Paris Club creditors have also committed to providing additional bilateral relief going beyond the HIPC Initiative.

5/ Includes project and program grants, as well as debt relief on interest payments.

6/ Reserves at current exchange rates. Includes balances in the special Bank for International Settlements. 
Table 7. Zambia: External Financing, 2000-07

(In millions of U.S. dollars)

\begin{tabular}{|c|c|c|c|c|c|c|c|c|c|}
\hline & \multirow{2}{*}{$\begin{array}{r}2000 \\
\text { Est. }\end{array}$} & \multirow{2}{*}{$\begin{array}{c}2001 \\
\text { Est. }\end{array}$} & \multirow{2}{*}{$\begin{array}{r}2002 \\
\text { Est. }\end{array}$} & \multirow{2}{*}{$\begin{array}{r}2003 \\
\text { Est. }\end{array}$} & \multicolumn{2}{|c|}{2004} & \multirow{2}{*}{$\begin{array}{l}2005 \\
\text { Proj. }\end{array}$} & \multirow{2}{*}{$\begin{array}{l}2006 \\
\text { Proj. }\end{array}$} & \multirow{2}{*}{$\begin{array}{l}2007 \\
\text { Proj. }\end{array}$} \\
\hline & & & & & Prog. & Proj. & & & \\
\hline Current account deficit (excl. official transfers and interest ) & -467 & -614 & -543 & -589 & -469 & -520 & -623 & -687 & -723 \\
\hline Change in n.f.a. of commercial banks & -89 & 40 & -53 & 48 & -5 & -90 & 17 & -10 & -10 \\
\hline Private capital (net) & 278 & 301 & 178 & 233 & 171 & 324 & 221 & 125 & 172 \\
\hline Gross reserves $(-=$ increase $)$ & -68 & 0 & -169 & 89 & 24 & -28 & -24 & -64 & -43 \\
\hline BoZ liabilities & -7 & -5 & -6 & -6 & -6 & -6 & -6 & 0 & 0 \\
\hline Other foreign assets of the $\mathrm{BoZ}$ & 30 & $\ldots$ & $\ldots$ & $\ldots$ & $\ldots$ & $\ldots$ & $\ldots$ & $\ldots$ & $\ldots$ \\
\hline Debt service, scheduled 1/ & -494 & -590 & -593 & -616 & -578 & -710 & -604 & -283 & -308 \\
\hline Interest & -155 & -144 & -137 & -131 & -120 & -121 & -110 & -101 & -95 \\
\hline Multilaterals & -39 & -37 & -31 & -33 & -33 & -34 & -31 & -28 & -26 \\
\hline Of which: IMF & -9 & -8 & -3 & -6 & -6 & -6 & -6 & -4 & -5 \\
\hline Bilaterals & -116 & -107 & -105 & -98 & -88 & -85 & -75 & -69 & -64 \\
\hline Of which: Paris Club & -114 & -105 & -104 & -97 & -88 & -82 & -73 & -67 & -63 \\
\hline Amortization & -340 & -446 & -456 & -486 & -458 & -588 & -494 & -182 & -213 \\
\hline Multilaterals & -41 & -260 & -270 & -295 & -292 & -347 & -348 & -114 & -142 \\
\hline Of which: IMF & 0 & -213 & -222 & -244 & -241 & -257 & -269 & -15 & -39 \\
\hline Bilaterals & -192 & -186 & -186 & -191 & -173 & -259 & -172 & -97 & -97 \\
\hline of which: Paris Club & -173 & -167 & -169 & -180 & -162 & -211 & -136 & -81 & -84 \\
\hline Other debt related items & 42 & -19 & 21 & -10 & -14 & 0 & 0 & 0 & 0 \\
\hline Accumulation of arrears $(+=$ increase $)$ & -10 & 31 & 12 & 48 & -60 & 0 & 0 & 0 & 0 \\
\hline Financing gap before debt relief and donor disbursements & -785 & -856 & -1153 & -804 & -937 & -1029 & -1020 & -919 & -912 \\
\hline Debt relief & 217 & 436 & 437 & 389 & 276 & 264 & 480 & 197 & 191 \\
\hline Before HIPC Initiative-Paris Club 2/ & 217 & 170 & 171 & 154 & 120 & 245 & 152 & 70 & 64 \\
\hline HIPC Initiative debt relief $3 /$ & $\ldots$ & 266 & 266 & 235 & 156 & 19 & 328 & 127 & 128 \\
\hline Of which: IMF & $\ldots$ & 150 & 153 & 169 & 0 & 2 & 237 & 8 & 4 \\
\hline Of which: Paris Club & $\ldots$ & 65 & 64 & 16 & 100 & -2 & 38 & 51 & 56 \\
\hline Gross official assistance & 457 & 527 & 685 & 416 & 661 & 668 & 540 & 606 & 611 \\
\hline Official creditors, excl. IMF & 431 & 433 & 512 & 416 & 425 & 421 & 505 & 574 & 596 \\
\hline Budgetary support & 185 & 75 & 138 & 55 & 69 & 65 & 133 & 101 & 82 \\
\hline World Bank 4/ & 140 & 44 & 56 & 20 & 34 & 21 & 20 & 20 & 20 \\
\hline African Development Bank & 13 & 0 & 13 & 0 & 9 & 0 & 24 & 15 & 18 \\
\hline European Union/Other 5/ & 32 & 31 & 69 & 35 & 26 & 44 & 89 & 66 & 44 \\
\hline Project financing & 246 & 358 & 347 & 341 & 356 & 356 & 373 & 473 & 514 \\
\hline Grants & 153 & 222 & 236 & 240 & 246 & 246 & 284 & 383 & 420 \\
\hline Loans & 93 & 136 & 111 & 101 & 110 & 110 & 89 & 90 & 93 \\
\hline Commodity support & 0 & 0 & 27 & 20 & 0 & 0 & 0 & 0 & 0 \\
\hline IMF (PRGF) & 26 & 94 & 173 & 0 & 236 & 248 & 34 & 32 & 16 \\
\hline Errors and ommissions & 111 & -107 & 31 & -2 & 0 & 97 & 0 & 0 & 0 \\
\hline Financing gap $(-=$ deficit $)$ & 0 & 0 & 0 & 0 & 0 & 0 & 0 & -117 & -109 \\
\hline \multicolumn{10}{|l|}{ Memorandum items: } \\
\hline Official debt service, cash payments, incl IMF & -245 & -142 & -123 & -189 & -376 & -446 & -124 & -86 & -117 \\
\hline Official debt service, cash payments, excl IMF & -237 & -70 & -50 & -108 & -129 & -185 & -86 & -76 & -77 \\
\hline Of which: Paris Club & -38 & -26 & -5 & -69 & -104 & -50 & -19 & -27 & -27 \\
\hline Net resource inflows, incl. IMF & 212 & 385 & 563 & 227 & 285 & 223 & 416 & 519 & 495 \\
\hline Percent of GDP & 7 & 11 & 15 & 5 & 6 & 4 & 7 & 10 & 9 \\
\hline Net resource inflows, excl. IMF & 195 & 363 & 462 & 308 & 296 & 236 & 420 & 498 & 519 \\
\hline Percent of GDP & 6 & 10 & 12 & 7 & 6 & 4 & 7 & 8 & 7 \\
\hline Gross official external reserves, eop & 114 & 114 & 283 & 194 & 173 & 222 & 246 & 310 & 353 \\
\hline Months of imports & 1.0 & 0.9 & 2.2 & 1.3 & 1.0 & 1.2 & 1.3 & 1.5 & 1.7 \\
\hline Percent of annual official debt service & 82 & 80 & 231 & 101 & 46 & 60 & 162 & 360 & 303 \\
\hline
\end{tabular}

Sources: Bank of Zambia (BoZ); and IMF staff estimates and projections.

1/ Scheduled, before debt relief. Drawn from 2000 decision point document adjusted for new loans.

2/ Indicates debt relief that would have been available under the traditional mechanism, relative to 1999 Paris Club rescheduling, comparable treatment from non-Paris Club bilaterals.

3/ The Paris Club has agreed to provide debt relief on Cologne flow terms through mid-2005. Once the completion point under the HIPC Initiative is reached, the Paris Club is expected to provide debt relief on Cologne stock terms. Paris Club creditors have also committed to providing additional bilateral debt relief beyond the HIPC Initiative.

4/ Assumes that Zambia moves to and remains on the base case for the Country Assistance Strategy.

5/ Assumes that disbursements under the EU variable tranche will be about 50 percent of funds allocated. 
Table 8. Zambia: Schedule of Disbursements Under the PRGF Arrangement, 2004-07

\begin{tabular}{|c|c|c|c|}
\hline \multicolumn{2}{|c|}{ Amount } & \multirow{2}{*}{$\begin{array}{l}\text { Availability } \\
\text { Date }\end{array}$} & \multirow[t]{2}{*}{ Conditions Necessary for Disbursement } \\
\hline $\begin{array}{l}\text { (In millions of } \\
\text { SDRs) }\end{array}$ & $\begin{array}{l}\text { (In percent } \\
\text { of quota) }\end{array}$ & & \\
\hline 82.5356 & 16.88 & June 16, 2004 & $\begin{array}{l}\text { Board approved the three-year arrangement and } \\
\text { endorsed the annual program. }\end{array}$ \\
\hline 82.5356 & 16.88 & December 15, 2004 & $\begin{array}{l}\text { Board completed the first review based on observance of } \\
\text { performance criteria for September 30, } 2004 \text {. }\end{array}$ \\
\hline 5.5024 & 1.13 & April 6, 2005 & $\begin{array}{l}\text { Observance of performance criteria for the December 31, 2004, } \\
\text { and completion of the second review. }\end{array}$ \\
\hline 5.5024 & 1.13 & June 16,2005 & Observance of performance criteria for the March 31, 2005. \\
\hline 5.5024 & 1.13 & September 13, 2005 & $\begin{array}{l}\text { Observance of performance criteria for the June } 30,2005 \text {, } \\
\text { and completion of the third review. }\end{array}$ \\
\hline 5.5024 & 1.13 & December 13, 2005 & Observance of performance criteria for the September 30, 2005. \\
\hline 5.5024 & 1.13 & March 15, 2006 & $\begin{array}{l}\text { Observance of the performance criteria for December } 31,2005 \text {, } \\
\text { and completion of the fourth review. }\end{array}$ \\
\hline 5.5024 & 1.13 & June 16, 2006 & Observance of performance criteria for the March 31, 2006. \\
\hline 5.5024 & 1.13 & September 13, 2006 & $\begin{array}{l}\text { Observance of performance criteria for the June } 30,2006 \text {, } \\
\text { and completion of the fifth review. }\end{array}$ \\
\hline 5.5024 & 1.13 & December 13, 2006 & Observance of performance criteria for the September 30, 2006. \\
\hline 5.5024 & 1.13 & March 14, 2007 & $\begin{array}{l}\text { Observance of performance criteria for the December } 31,2006 \text {, } \\
\text { and completion of the sixth review. }\end{array}$ \\
\hline 5.5024 & 1.13 & May 31,2007 & Observance of performance criteria for the March 31, 2007. \\
\hline
\end{tabular}


Lusaka, March 23, 2005

Mr. Rodrigo de Rato

Managing Director

International Monetary Fund

Washington, D.C.

Dear Mr. de Rato,

The first review of the economic program supported by Zambia's arrangement with the Fund under the Poverty Reduction and Growth Facility (PRGF) was completed on December 15, 2004. In the attached Memorandum of Economic and Financial Policies (MEFP) and Technical Memorandum of Understanding, we review progress in implementing the program in 2004 and update the program for 2005. The focus of our strategy continues to be on achieving high rates of economic growth and reducing poverty through sustainable fiscal and monetary policies and structural reforms to support fiscal adjustment and a reorientation of spending toward poverty reduction, improve the environment for private sector development, and strengthen governance.

The Government of Zambia requests the completion of the second review and the third disbursement under the PRGF arrangement in the amount of SDR 5.502 million. In this connection, the Government also requests the IMF Executive Board to grant waivers for the nonobservance of two performance criteria. A delay in making debt service payments to Russia resulted in nonobservance of the performance criterion on the accumulation of external payments areas. These payments have now been settled. The government also requests a waiver for the nonobservance of the performance criterion on cabinet approval of a proposal to repeal sections of the Building Societies Act, the National Savings and Credit Bank Act and the Development Bank of Zambia Amendment Act that are conflict with the Banking and Financial Services Act. The government expects that cabinet will approve the proposal in March, as a prior action for completion of the review. We are also requesting modifications to the quantitative performance criteria for 2005.

The Government of Zambia believes that the successful pursuit of the economic program supported by the PRGF arrangement, approved on June 16, 2004, along with the diligent implementation of the Poverty Reduction Strategy Paper in 2004 and the good progress that has been made in meeting the completion point triggers, has laid an excellent basis for us to attain the completion point under the enhanced Highly Indebted Poor Countries (HIPC) Initiative before the end of March, 2005. The debt relief to be provided under the HIPC 
Initiative will certainly assist Zambia in reaching its growth and poverty reduction objectives. To make steady progress toward the Millennium Development Goals, Zambia will, however, need additional financial assistance from the international community.

The Government of Zambia believes that the policies set forth in the attached MEFP are adequate to achieve the objectives of its program, but will take any further measures that may become appropriate for this purpose. Zambia will consult with the Fund on the adoption of these measures and in advance of revisions to policies contained in the MEFP, in accordance with the Fund's policies on such consultation.

The performance criteria and benchmarks for the third review, which is scheduled to be completed in mid-September 2005, will be based on June 2005 targets, and the performance criteria and benchmarks for the fourth review, which is scheduled to be completed in midMarch 2006, will be based on December 2005 targets, as set out in Tables 3 and 4 of the MEFP.

The Government of Zambia authorizes the Fund to make this letter and the attached memoranda available to the public, including through the placement of these documents on the Fund's website, subject to the removal of market sensitive information, following the approval of the Fund's Executive Board of the completion of the second review under the PRGF arrangement.

Yours sincerely,

$/ \mathrm{s} /$

Ng'andu P. Magande, MP

Minister of Finance and National Planning 


\section{ZAMBIA}

\section{Memorandum of Economic and Financial Policies of the Government of Zambia for 2005}

\section{RECENT ECONOMIC DEVELOPMENTS}

\section{A. Economic Performance in 2004}

1. Macroeconomic performance continued to strengthen in 2004, surpassing expectations. Real GDP growth is now estimated to have reached at least 5 percentcompared to the original budget assumption of 3.5 percent - and, despite the sharp increase in fuel prices during the year, the 12-month rate of inflation was contained at 17.5 percent. Strong growth in construction and mining contributed to this favorable outturn, while agricultural output expanded by just over 6 percent.

2. Fiscal policy implementation was broadly in line with the program, resulting in a sharp decline in domestic borrowing. In the final quarter of the year, tax collections strengthened, limiting the shortfall in tax and non-tax revenues to 0.3 percent of GDP. Total spending was 1 percent of GDP less than programmed, mainly reflecting a slower rate of execution of capital spending financed by external project grants and loans. Domestically financed spending (including interest payments) was 0.2 percent of GDP less than programmed. Within this total, the wage bill was 7.8 percent of GDP, as budgeted. Recurrent departmental charges (RDCs) were 0.5 percent of GDP higher than originally budgeted, as a result of supplementary allocations during the second half of the year. These included an increase in military spending and payments made by central government to settle arrears of central and local government to water utilities and ZESCO, as called for in the commercialization strategy for the electricity utility. This higher spending was more than offset by lower interest payments and savings on non priority capital projects. However, government protected releases for expenditures on priority poverty reducing programs which, for the first time in three years, were fully funded as budgeted. Improved fiscal discipline, and the delivery of external budget support in line with program expectations, allowed for a substantial reduction in government's domestic borrowing from over 5 percent of GDP in 2003 to less than 1 percent of GDP in 2004. However, authorized expenditure of about 1 percent of GDP went unexecuted by the end of the year, mainly because more than half of the budget support expected in 2004 (US\$38 million out of US\$65 million) came only in the last few days of December 2004.

3. The Bank of Zambia began to tighten liquidity conditions in mid-year but, as a result of exceptional factors, monetary growth surged at the end of 2004. Through November, the growth of reserve and broad money was 5 percent and 22 percent respectively. However, partly as a result of the large end-year disbursements of donors' budget support and transfers from the Government's accounts in the Bank of Zambia (BoZ) 
to its accounts in commercial banks, reserve money rose sharply to bring the end-year increase to 21 percent. Similarly, the 12-month increase in broad money reached 30 percent in December. Large foreign currency deposits by corporations into commercial bank accounts also contributed significantly to the rise in broad money. Interest rates on treasury bills, which had fallen very sharply, from over 30 percent in December 2003 to about 7 percent in June 2004, began to rise in mid-year and stabilized in the final quarter at about 18 percent.

4. Zambia's external position strengthened in 2004. Proceeds from copper and other metal exports increased by 65 percent as a result of a solid rise in metals production and an increase of copper prices in excess of 50 percent. The growth of non-metals exports, notably cotton lint and tobacco, also exceeded expectations. Thus, despite an increase in imports of 24 percent, owing mainly to an increase in capital imports for the mining sector and high oil prices, the current account deficit (including grants) narrowed from 7.5 percent of GDP in 2003 to an estimated 4.8 percent in 2004. With somewhat lower-than-projected external debt service payments and larger than anticipated purchases by the BoZ, the buildup of gross international reserves during the year exceeded the program target by US\$35 million; by year-end, gross international reserves reached US\$222 million, for an import coverage of 1.2 months, compared with the program target of 1.1 months. The kwacha appreciated against the dollar during the final quarter of 2004, and for the year as a whole the real effective exchange rate of the kwacha appreciated by about 8 percent.

5. The Government has finalized its second PRSP Annual Progress Report (APR), covering the period July 2003 to June 2004. The Government has also prepared an Addendum to the second APR, which covers progress in the implementation and monitoring and evaluation of Zambia's poverty reduction strategy from July to December 2004 and a supplement to the APR which lays out objectives and policies for 2005.

\section{B. Performance Under the Program Supported by the PRGF Arrangement}

\section{All but two of the quantitative and structural performance criteria (PCs) for the} second review were observed, as were all quantitative benchmarks (Tables 1 and 2). In early February, the government settled payments due to Russia that had been due since October. Under the structural program, the PC for January 2005 regarding the adoption of the work program for the Public Expenditure Management and Financial Accountability (PEMFA) reform program was met. The structural performance criterion regarding cabinet approval of a proposal to repeal sections of the Building Societies Act, the National Savings and Credit Bank Act, and the Development Bank of Zambia Act that were in conflict with the Banking and Financial Services Act was not met. This measure is a prior action for the completion of the second review. In addition, Government has continued to refrain from making extra budgetary payments for which it is not legally obligated (a continuous PC), and observed the limits on funding unforeseen expenditures (a continuous benchmark). Quarterly budget execution reports were published, but with a delay of more than the 45 days specified in the benchmark for the third quarter report. Government developed a wider definition of poverty reducing spending that was used in the preparation of the budget. Consultations are 
being held with stakeholders to confirm that this definition adequately reflects the priorities set out in the government's PRSP.

\section{THE MEDIUM-TERM STRATEGY}

7. The Government has reaffirmed the strategy set out in the PRSP and the November MEFP to promote growth through diversification while improving the quality of public services. Key macroeconomic objectives include real GDP growth of at least 5 percent a year over the medium term, with annual inflation falling to single digits by 2007 . In line with the objective of reducing domestic debt to sustainable levels, the framework calls for a gradual reduction in Government's domestic borrowing over the medium term. Government remains committed to increasing poverty-reducing spending by at least 1.6 percent of GDP between 2004 and 2007 .

8. As explained in the November 2004 MEFP, the medium term expenditure framework now envisages considerably higher spending to conduct the elections in 2006 and for the constitutional review. Election related expenditures in 2005 are fully funded in the budget. The government recognizes that the remaining financing gaps in 2006 and 2007 will need to be filled through a combination of additional grants and offsetting measures, including some reduction in the costs of these processes. A technical working group headed by the Ministry of Justice with representatives of the Electoral Commission of Zambia and donors is examining cost estimates, drawing on international experience. At this stage, no firm commitments have been made by donors to the government's request for additional assistance.

\section{THE PROGRAM FOR 2005}

9. The Government's economic policy agenda for 2005 also remains essentially unchanged from that presented in the November MEFP. Owing to the sharp increase in the monetary aggregates in late 2004 , however, some adjustments to the monetary program have been necessary to maintain the objective of lowering inflation to 15 percent by end2005. In addition, quantitative program targets have been set for the second half of 2005 and new structural measures have been introduced for the remainder of the year in the areas of public expenditure management and financial sector development.

\section{A. Fiscal Policy}

10. The budget proposal submitted to Parliament aims to limit domestic financing needs to 1.6 percent of GDP, in line with the framework included in the MEFP of November. Aggregate revenues are expected to be 18.4 percent of GDP, or 0.1 percent of GDP higher than in the earlier framework. The combination of these higher revenues and the Government's decision to cancel a census costing 0.1 percent of GDP, which had been included in initial spending plans for the constitutional review, will offset increases in other spending, including a small increase in the wage bill. The Ministry of Finance and National Planning (MoFNP) and the BoZ will closely monitor an expected partial unwinding of the 
balances at end-December 2004 of government deposits in the BoZ and commercial bank accounts and will ensure its consistency with the program objectives. To accommodate this unwinding in the program, the Technical Memorandum of Understanding (paragraphs 5 and 9) describes an adjustor to net domestic financing of the central government and to net domestic assets of the Bank of Zambia. Additional domestic financing up to 0.3 percent of GDP will be available provided that domestic revenues and expenditures are in line with the budget. The government also reaffirms the commitment to limit the need to authorize supplementary budget allocations during the year to enhance fiscal policy credibility.

11. The budget includes measures aimed at easing the burden of direct taxation, while broadening the tax base to raise revenues. The revised personal income tax regime, effective April 1, 2005, increases the threshold for exempting annual income to K 3.4 million (from K 3.1 million) and reduces the highest tax rate of 40 percent to 37.5 percent. These measures are expected to lead to a revenue loss of $\mathrm{K} 35.7$ billion or 0.1 percent of GDP. Several agricultural products, previously exempted, are now standard rated under the VAT regime; when exported, these products will attract VAT at a zero rate, resulting in a revenue loss of K 29.8 billion or 0.1 percent of GDP. Medical supplies and drugs that were previously exempted will be also be zero-rated, yielding a revenue loss of $\mathrm{K} 2.3$ billion. In line with the terms of the recent sale to a strategic investor, the duty free importation of consumables by Konkola Copper Mines will expire on March 31, 2005, yielding K 62.5 billion or 0.2 percent of GDP. In the area of customs duties and excises, several measures have been introduced, including a 25 percent export tax on raw-timber and scrap metal. These two measures are expected to yield K 24.6 billion. Higher customs duties on a few agricultural products should yield an additional K 5.8 billion. Overall, the tax policy measures are expected to raise revenues by 0.1 percent of GDP.

12. The Government recognizes that containing the wage bill continues to be critical for maintaining sound public finances and macroeconomic stability. The budget for 2005 limits the wage bill to 8.1 percent of GDP compared with 7.9 percent of GDP envisaged in the November MEFP. This increase will accommodate the hiring of additional teachers in 2005. It had been expected that the payment of terminal benefits to teachers using a grant from the Government of the Netherlands would enable the removal of 7,000 inactive teachers from the payroll, thereby making room for an equivalent number of new hirings. However, an audit identified fewer inactive teachers to be removed from the payroll and consequently additional resources were needed to bring total hirings to 7,000. The Ministry of Health has also been allocated resources to hire nearly 1,455 frontline staff in 2005 and to provide for a retention scheme for nurses and clinical staff.

13. The budget for $\mathbf{2 0 0 5}$ includes an allocation of $\mathrm{K} 343$ billion for wage increases. In line with procedures established in 2003, a government team comprised of representatives of the cabinet office and key ministries has commenced negotiations with unions representing government employees. In order to ensure consistency with the budget envelope, no wage agreements will be concluded outside the budget allocation of K 343 billion and this allocation will cover all public service employees. 
14. In the 2005 budget, the Government has broadened the definition of povertyreducing spending to more closely correspond to the priorities set out in Zambia's PRSP. Under the new definition, poverty reduction spending now includes a significant share of recurrent spending, including the wages of teachers in basic schools and health workers. On this definition 22.3 percent of domestically financed outlays, including external debt amortization, or the equivalent of 4.9 percent of GDP, are for poverty reduction. More than 80 percent of externally financed project expenditures, are identified as poverty reducing. It is expected that the budget for 2005 will entail an increase in poverty reducing expenditures of at least 0.5 percent of GDP as had been envisaged using the narrow definition. The reclassification of 2004 expenditures using the new definition, which is needed for comparison with the 2005 budget, will be available only after government has, in consultation with stakeholders, formally adopted a revised definition of poverty reducing spending.

15. The introduction of activity-based budgeting (ABB) has also allowed a more appropriate classification of programs under capital and recurrent expenditures. As a result in the 2005 budget, RDCs and transfers and pensions have increased by 1.6 percent and 1.9 percent of GDP respectively from the November MEFP, while capital spending and the residual category of other recurrent expenditures have declined by slightly more than the corresponding total amount.

\section{The Government gives high priority to the settling of domestic arrears on} statutory payments to public sector employees and to suppliers of goods and services. The 2005 budget allocates K 65 billion to the payment of arrears on housing allowances, sufficient to pay all remaining arrears from 2002 and a third of the outstanding arrears from 2003. The Government will also repay K 159 billion of pension arrears to public sector employees. In addition, the budget allocates $\mathrm{K} 76$ billion toward the payment of outstanding bills owed to contractors and suppliers, amounting to K 548 billion at end-September 2004 billion, including high penalty interest. As described in the November MEFP the government is seeking to negotiate discounts on these arrears to contractors and suppliers in an orderly and equitable framework. The government is also seeking donor assistance to settle these arrears and is considering the option of issuing medium-term bonds for this purpose. In total, the 2005 budget allocates K 300 billion for clearing arrears.

\section{B. Monetary Policy}

\section{The monetary program for 2005 has been tightened to reverse the} unanticipated increase in reserve and broad money at the end of 2004. In January, the BoZ mopped up excess liquidity, withdrawing over K 237 billion from banks' excess reserves and reversing most of the build up in reserve money at the end of 2004. For the year as a whole, the growth in reserve money will be limited to 10.5 percent, with the aim of containing the growth in broad money to 14.8 percent. These growth rates imply that by end2005 , stocks of reserve and broad money will be in line with the targets established in the November MEFP. Despite this slowing of monetary growth, the program will accommodate an expansion of credit to the private sector in real terms as commercial banks are expected to 
unwind the extraordinary buildup of foreign assets during December 2004, part of which has been identified as temporary in nature.

\section{External Prospects for 2005}

18. The external outlook for $\mathbf{2 0 0 5}$ is favorable. Notwithstanding an anticipated easing of copper prices from their record levels of 2004, metals exports are expected to rise by 17 percent, owing to a jump in copper production from the coming on stream of the Kansanshi copper mine. Non-metals exports are also expected to continue to record healthy growth. With work on the new Lumwana mine intensifying, imports to the mining sector are likely to remain close to their 2004 level. The oil import bill is also likely to remain high as a result of continued high oil prices and the need to restore inventories drawn down in 2004 . Total imports are therefore projected to rise by 4 percent in U.S. dollar terms. On this basis, the current account deficit (including grants) is projected to narrow further, to 3.1 percent of GDP in 2005.

19. Donor support and anticipated debt relief after the completion point ensure that the 2005 program is fully financed. External budgetary support is now projected to be $\$ 133$ million, or about $\$ 15$ million higher than at the time of the first review; about twothirds of the support is expected to be in the form of grants. At the same time, debt service obligations are estimated to be $\$ 15$ million higher than at the time of the first review. Based on the experience to date, disbursements under the EU's PRBS for 2004-06 are now expected to be received somewhat earlier than before. Good progress has been made in meeting the conditions for the release of the second tranche of the World Bank's structural adjustment credit. The program does not factor in a possible increase in bilateral grants under the Wider Harmonization in Practice (WHIP) program. Multilateral debt relief after the achievement of the completion point under the HIPC Initiative will contribute importantly to the financing of the 2005 program.

20. Zambia's trade regime remains open and supportive of export development and the diversification of the economy. The changes in tariff rates announced with the 2005 budget are minor and have a limited impact on the average nominal tariff on imports, estimated at 11.5 percent. The 25 percent tax on the export of unprocessed timber and scrap metals serves both the purpose of protecting the environment and creating additional employment in certain localities; however, the macroeconomic impact of these taxes, which replace bans on the export of timber and scrap metal, will be minimal. The Government is reviewing the experience of participating in the COMESA free trade area since 2000 and its options regarding a common external tariff. Based on this review, the feasibility of Zambia's membership of a COMESA customs union will be assessed.

\section{Structural Measures}

21. Structural reforms in $\mathbf{2 0 0 5}$ will continue to focus on public expenditure management and financial accountability (PEMFA), financial sector reform-especially the resolution of three non-bank financial institutions - and governance. These reforms, 
which are critical to support macroeconomic stability and growth, were elaborated in the last MEFP. The focus will therefore be on areas in which additional steps have been defined in the program

\section{Public Expenditure Management}

22. The work program for the first year of PEMFA, developed with cooperating partners supporting the program, was adopted in January 2005. Initial disbursements to support activities under this five year program totaling US\$72 million are expected to commence in the first quarter following the adoption of procurement guidelines. Against this background, priority under the PRGF-supported program is to be given to key steps needed to support the implementation of the PEMFA program or areas in which preparatory work is already well advanced. Thus the government will by end-May finalize, in consultation with the PEMFA technical working group, a framework for monitoring and evaluating the PEMFA program. This will draw upon the benchmarks of public expenditure management that are used under the HIPC assessment and action plans to track poverty-reducing spending (HIPC AAP) and the Public Expenditure and Financial Accountability (PEFA) framework. It is expected that the first assessment under this framework will be conducted by November 2005. To further strengthen the commitment control system, the MoFNP, in consultation with the PEMFA technical working group will by end-May design a cash-flow framework for all line ministries. Following the passage of the Finance Act, the government will by September 2005 prepare and issue new financial regulations and revised accounting manuals. By end-August 2005, the Budget Office in the MoFNP will prepare and publish the first draft (Green Paper) of the Medium-Term Expenditure Framework for 2006-08 for consultation with stakeholders. The Government will refrain from paying any amounts for which it is not legally liable and which are not included in the budget. The Government continues to publish quarterly budget execution reports using the activity-based budgeting classification, within 45 days of the end of each quarter. Finally, unbudgeted expenditure requirements will be funded only to the limits of the contingency resources indicated in the budget or only after the Cabinet has approved any changes by finding compensatory funding within the approved budget resources.

\section{The IFMIS component of the PEMFA work program has incurred further} delays, arising from complications beyond the Government's control. After Government modified the contract for the installation of IFMIS hardware and software to address issues raised by World Bank staff, it was learned that the contractor had separated from its parent company and changed its name. This action brought into question whether the new independent firm has the capacity to assure good execution of the contract. As proposed by the World Bank, the Government is exploring the possibility of the firm entering a joint venture with its former parent, in order to address the capacity question and to move ahead with the pilot project as soon as possible. As at the time of the first review, it is anticipated that the piloting of the IFMIS in at least three ministries will begin in June 2005. The Government will report on the findings of a preliminary review of the piloting of the IFMIS in at least three line ministries by end-December 2005. 
24. Special audits of the military wage bill and RDCs are being conducted by the auditor general. The increase in the budget allocation to the defense forces in 2005 has been limited to 11 percent. Additional steps will be taken in the light of the findings of these audits, which are expected to be presented to the President of Zambia in April.

\section{The Government is committed to strengthening Zambia's debt management}

capacity. The Government recognizes that its framework and capacity for the management of both external and domestic debt are inadequate in many respects and is actively working with the World Bank under their Debt Reform and Capacity Building Program to design a work program that will address the deficiencies. As part of this program, the Government has committed itself to the preparation of a public debt management reform plan. This plan will involve the establishment of an institutional framework that will centralize the management of debt and borrowing strategy in the Investment and Debt Management Department (IDM) of the MoFNP. A central and immediate need in this area is the continuous maintenance of an updated debt database as is the strengthening of a back office function to ensure the accuracy and comprehensiveness of debt transaction records and punctual payments on outstanding debt based on a reliable debt recording system. Also pressing is the need to strengthen the capacity of the IDM to conduct basic risk analysis to underpin the formulation of a debt management strategy, as well as to provide input into post-completion point borrowing negotiations. The establishment of a best practices debt management framework capacity will require resources and take considerable time. As a first step, the Government has decided that, beginning with the second quarter of 2005, IDM will validate the stock of government's external debt on a quarterly basis. Within 45 days of the end of each quarter IDM will provide the budget office with an updated projection of debt service due over the following three years. From August 2005, this will be a continuous performance criterion under the PRGF-supported program.

\section{Financial Sector Developments}

26. In line with the strategy laid out in the November 2004 MEFP (paragraph 35), the government took measures to address three problem non-bank financial institutions (NBFIs). It is expected that cabinet will shortly approve proposals - drafted with technical assistance from the IMF - to repeal sections of the National Savings and Credit Bank Act, the Development Bank of Zambia Amendment Act, and the Building Societies Act which are in conflict with the Banking and Financial Services Act. As a result of these amendments, the regulatory and supervisory powers of the BoZ over NBFIs will be strengthened.

\section{Progress was also made under the action plans of the National Savings and} Credit Bank (NSCB) and the Development Bank of Zambia (DBZ), as set out in the Memoranda of Understanding (MoU) signed in November. Performance of both the NSCB and the DBZ improved in the last quarter of 2004. Under the transitional provisions of the $\mathrm{MoU}$, the $\mathrm{BoZ}$ is exercising strengthened supervisory and regulatory powers over these two institutions. In observance of its commitments to contribute to the recapitalization of the NSCB and following IFAD's decision to participate in the recapitalization plan, the Government cancelled the NSCB's outstanding obligations of K 5.3 billion to the Zambia 
Revenues Authority, National Pension Scheme Authority, and the Zambia National Building Society. The Government will inject K 5.8 billion in cash into NSCB, when IFAD's contribution has been received. As detailed in the November MEFP, the DBZ received a capital injection by Ex-Im Bank of India amounting to 34 percent of its total paid up capital. With technical assistance from cooperating partners, DBZ and the NSCB will by June and September 2004, respectively, prepare business plans leading to full compliance with the BFSA and their incorporation under the Companies Act in 2006. The NSCB and the DBZ will submit incorporation plans to the BoZ by end-December 2005 (structural benchmark). In February, the BoZ received and assessed a revised business plan prepared by the Zambia National Building Society (ZNBS). To protect depositors' funds and ensure reduction of operating costs, the BoZ is continuing to monitor ZNBS's operations. By end-April, the Government and the BoZ, in consultation with the staffs of the World Bank and the IMF, will adopt an action plan for the resolution of the ZNBS.

28. The Government is committed to concluding the sale of 49 percent of the equity in the Zambia National Commercial Bank (ZNCB) to a strategic investor. To this end, the Zambia Privatization Agency (ZPA), following an international tender, selected a preferred and a reserve bidder to conduct detailed negotiations. The preferred bidder was the Amalgamated Bank of South Africa (ABSA) Group; the reserve bidder was the Africa International Financial Holdings Zambia Limited (AIFHZL). Negotiations with ABSA commenced in September 2003. Although negotiations were substantially concluded with the ABSA negotiation team in August 2004, and despite being given extended time, the ABSA team has failed to secure the approval of their board. In the meantime, in follow-on negotiations with AIFHZL, the ZPA concluded that a satisfactory agreement could not be reached. Apart from the many important new issues that arose after the submission of their bid, the AIFHZL consortium's membership changed raising questions and creating uncertainties about the group's final composition. In light of these developments and the long delays in concluding agreement with the two bidders, Government terminated negotiations. ZPA, will issue, in late March or early April as soon as procedural requirements are met, a new invitation for bids for purchase of the 49 percent equity in ZNCB with management control, in line with Government's privatization and financial sector policies. (This step constitutes a structural performance criterion for end-May). Given the preliminary interest exhibited by some reputable banks operating in the region, it is expected that the selection of new bidders and ensuing negotiations would proceed at a rapid pace. The sale of an additional 25.8 percent of shares in ZNCB to the Zambia Privatization Trust Fund will also proceed.

\section{Private sector development}

\section{The Government has taken several steps to advance the private sector} development initiative (PSDI). Priorities and institutional arrangements are being established to support the implementation of the initiative, which requires the cooperation and coordination of numerous ministries. In February, the Domestic Business Council (chaired by the President) and the PSDI steering committee (chaired by the deputy secretary to the cabinet) met for the first time. These groups bring together Government officials and 
representatives of the business and donor communities to monitor implementation of the PSDI work plan. It is expected that donor assistance will be forthcoming to support this effort. Alongside these steps to put in place an implementation mechanism, initial steps are being focused on areas that are directly under the control of the Ministry of Commerce Trade and Industry, the lead agency in the initiative. Proposals for revisions of the Trade Licensing Act, which would streamline trade licensing procedures, will be submitted to parliament; with approval, implementing regulations could then be established by end-2005. A new proposal to amend the Investment Act, that does not contain tax incentives, will be submitted to Cabinet during 2005. The new proposal would make operational a one-stop center to reduce bureaucratic procedures for investors to obtain required authorizations. The Government intends to establish the Tariff Commission, which includes among others the Ministers of Commerce, Finance and National Planning, and Justice, and representatives of the private sector to review tariff issues.

\section{TECHNICAL ASSISTANCE NEEDS}

30. The Government of Zambia and the BoZ have requested further technical assistance from the IMF to strengthen liquidity management operations of the BoZ; to strengthen the Bank of Zambia Act and legislation on the supervision of banks and other financial institutions; to prepare new regulations and revised accounting manuals for the new Finance Act; to enable compliance with the manual for Government Finance Statistics (2001); and to improve data on the monetary survey (follow-up). In addition, the Central Statistical Office will request follow-up technical assistance for the rebasing of data for the national accounts and consumer price index.

\section{PROGRAM MONITORING}

31. Progress in implementing the PRGF-supported program will continue to be monitored quarterly, based on the qualitative and structural performance measures indicated in Tables 3 and 4. Performance criteria are now proposed through the end of 2005. In addition, the government is requesting modifications to the performance criteria for March and June on net domestic assets of the BoZ and net domestic financing of the Government (and the inclusion of related adjustors), and a modification to the quarterly ceilings on the central government wage bill. These targets are defined in the attached technical memorandum of understandings. 
MEFP: Table 1. Zambia: Quantitative Performance Criteria (PC), Benchmarks and Indicative Targets Under the PRGF Program 1/ (In billions of Kwacha, unless otherwise indicated)

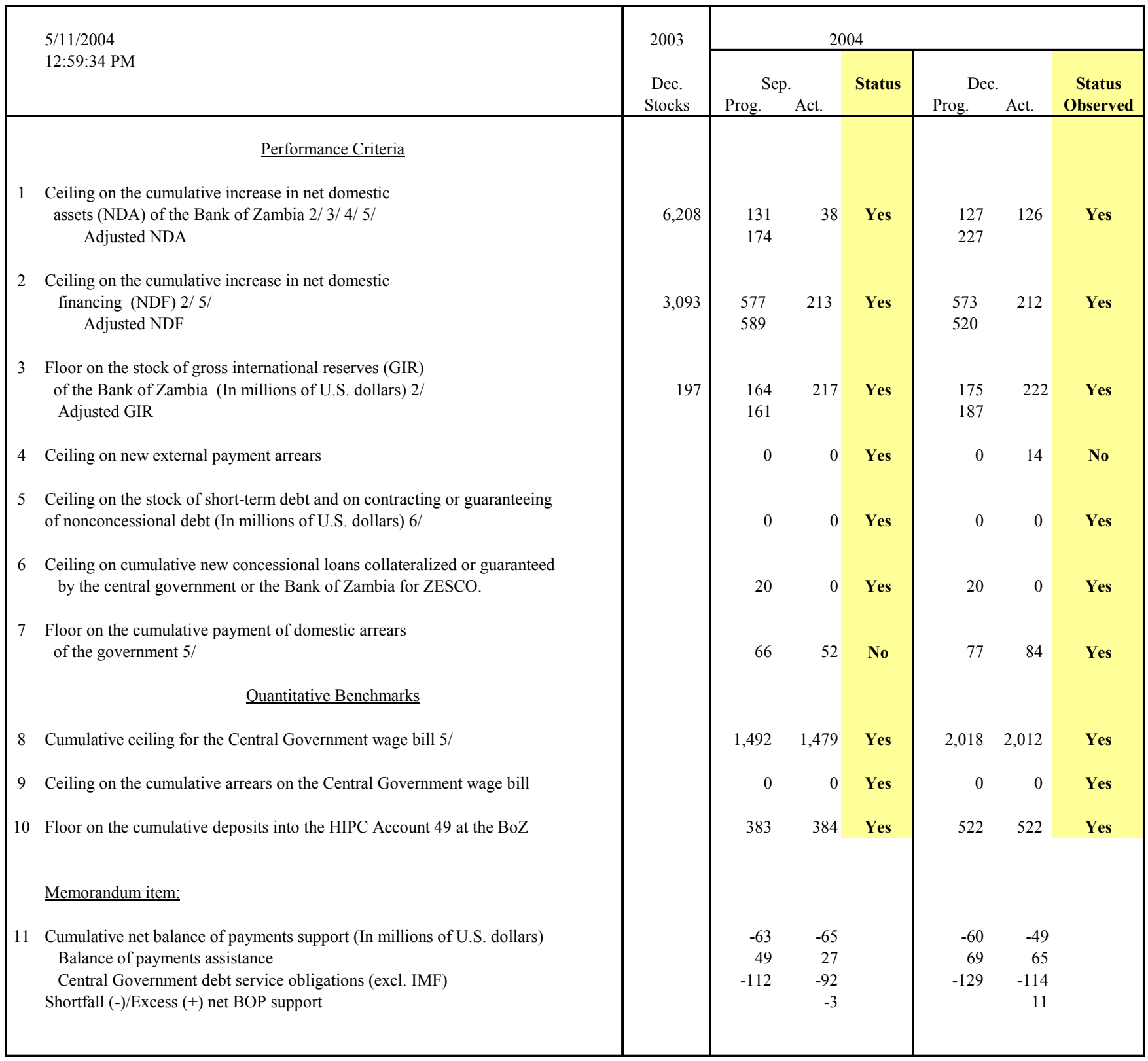

1/ The definitions of the indicative targets are contained in the Technical Memorandum of Understanding (TMU).

2/ Adjustors, including for balance of payments support are defined in the TMU.

3/ Excludes HIPC debt relief from the IMF.

4/ The ceiling will be adjusted for changes in the legal reserve requirements.

$5 /$ Cumulative from the end of 2003.

6/ Nonconcessional loans are defined as having a grant element of less than 40 percent. 
MEFP: Table 2. Zambia: Prior Actions, Structural Benchmarks, and Performance Criteria 1/

\begin{tabular}{|c|c|c|}
\hline & Timing & Status \\
\hline $\begin{array}{l}\text { Prior Action 2/ } \\
\text { Adoption by the government and the Bank of Zambia of action plans, finalized in consultation with the World Bank and IMF staff, for } \\
\text { the resolution of the National Savings and Credit Bank and the Development Bank of Zambia. }\end{array}$ & & Observed \\
\hline $\begin{array}{l}\text { Benchmarks and Performance Criteria } \\
\text { 1. Public Expenditure Management }\end{array}$ & & \\
\hline The Government will refrain from paying any amounts for which it is not legally liable and which are not included in the budget. 3/ & Continuous & Observed \\
\hline $\begin{array}{l}\text { Publication of quarterly budget execution reports using the activity-based budgeting classification, within } 45 \text { days of the end of each } \\
\text { quarter. }\end{array}$ & Continuous & $\begin{array}{l}\text { Not observed. End-Sept } \\
\text { data posted on the website } \\
\text { on Dec } 29 \text {. }\end{array}$ \\
\hline Adopt a work program covering the first year of the implementation of the PEMFA reform program. 3/ & Jan 2005 & Observed \\
\hline $\begin{array}{l}\text { Adopt a definition of poverty reducing spending corresponding to the priorities of the government PRSP and use this in the } \\
\text { preparation of the budget for } 2005 \text {. }\end{array}$ & Jan 2005 & Observed \\
\hline Initiate the piloting of the IFMIS in at least three line ministries. 3/ & June 2005 & On going \\
\hline $\begin{array}{l}\text { Report on the findings of a preliminary review of the piloting of the IFMIS in at least three line ministries. } 3 \text { / } \\
\text { 3. Financial Sector Reform }\end{array}$ & Dec 2005 & On going \\
\hline $\begin{array}{l}\text { Cabinet approval of a proposal to repeal the sections of the Building Societies Act, the National Savings and Credit Bank Act and the } \\
\text { Development Bank of Zambia Amendment Act that are in conflict with the Banking and Financial Services Act. 3/ }\end{array}$ & Feb 2005 & $\begin{array}{l}\text { Not observed. Prior Action } \\
\text { for the second review }\end{array}$ \\
\hline $\begin{array}{l}\text { Adoption by the Government and the Bank of Zambia of an action plan, finalized in consultation with the World Bank and IMF staff, } \\
\text { for the resolution of the Zambia National Building Society. } \\
\text { 4. Governance and Transparency }\end{array}$ & Apr 2005 & On going \\
\hline $\begin{array}{l}\text { Unforeseen expenditures will be funded only to the limits of the budget contingency or after Cabinet approval including the } \\
\text { identification of savings elsewhere in the budget. }\end{array}$ & Continuous & Observed \\
\hline
\end{tabular}

1/ The definitions of the prior actions, structural benchmarks, and performance criteria are contained in the Technical Memorandum of Understanding (TMU).

2/ The prior action for the completion of the review should be carried out and reported at least five working days prior to the Executive Board discussion.

3/ Performance criteria. 
MEFP: Table 3. Zambia: Quantitative Performance Criteria (PC), Benchmarks and Indicative Targets Under the PRGF Program 1/ (In billions of Kwacha, unless otherwise indicated)

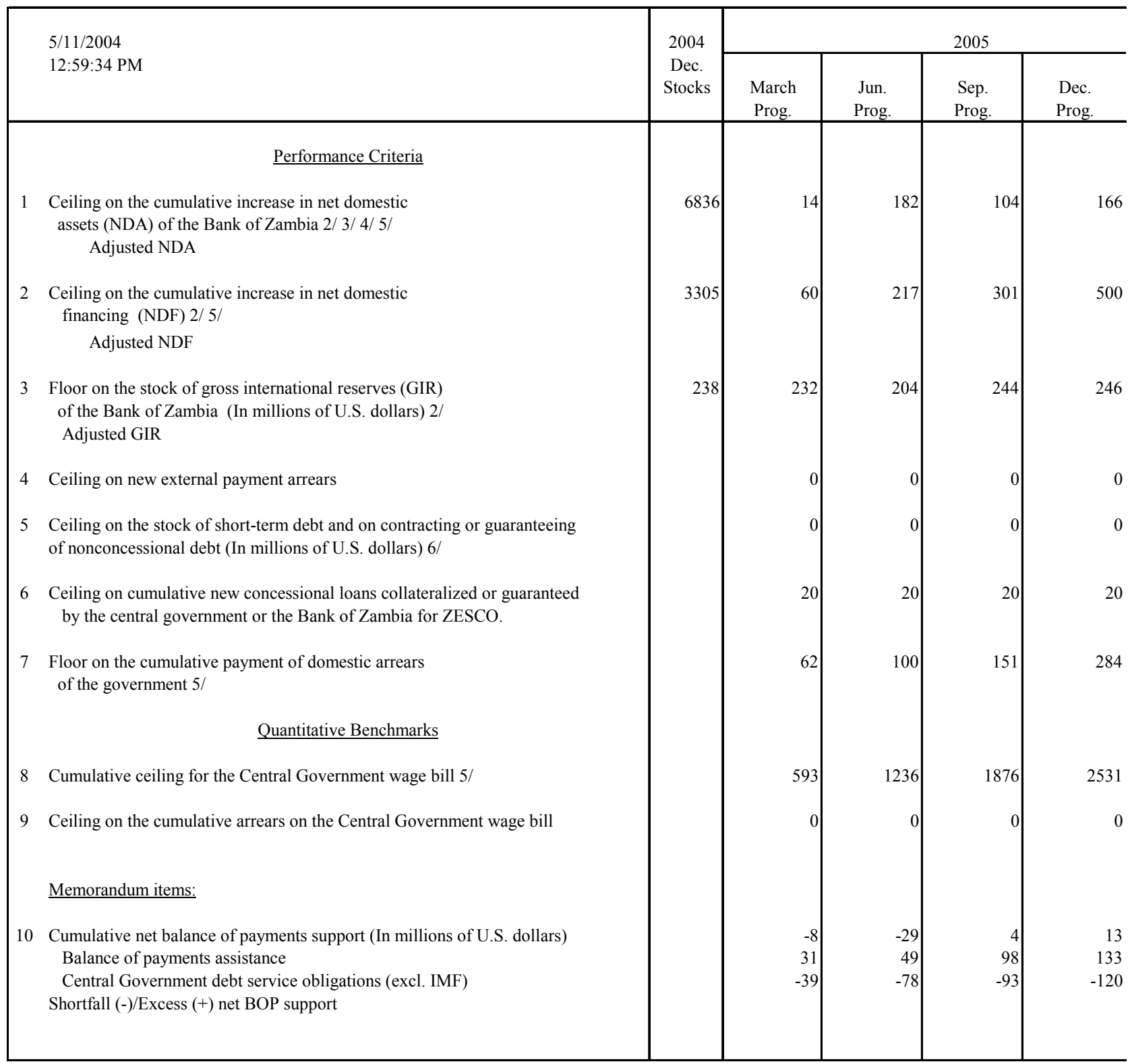

1/ The definitions of the indicative targets are contained in the Technical Memorandum of Understanding (TMU).

2/ Adjustors, including for balance of payments support are defined in the TMU.

3/ Excludes HIPC debt relief from the IMF.

4/ The ceiling will be adjusted for changes in the legal reserve requirements.

5/ Cumulative from the end of 2004.

6/ Nonconcessional loans are defined as having a grant element of less than 40 percent. 
MEFP: Table 4. Zambia: Structural Benchmarks and Performance Criteria 1/

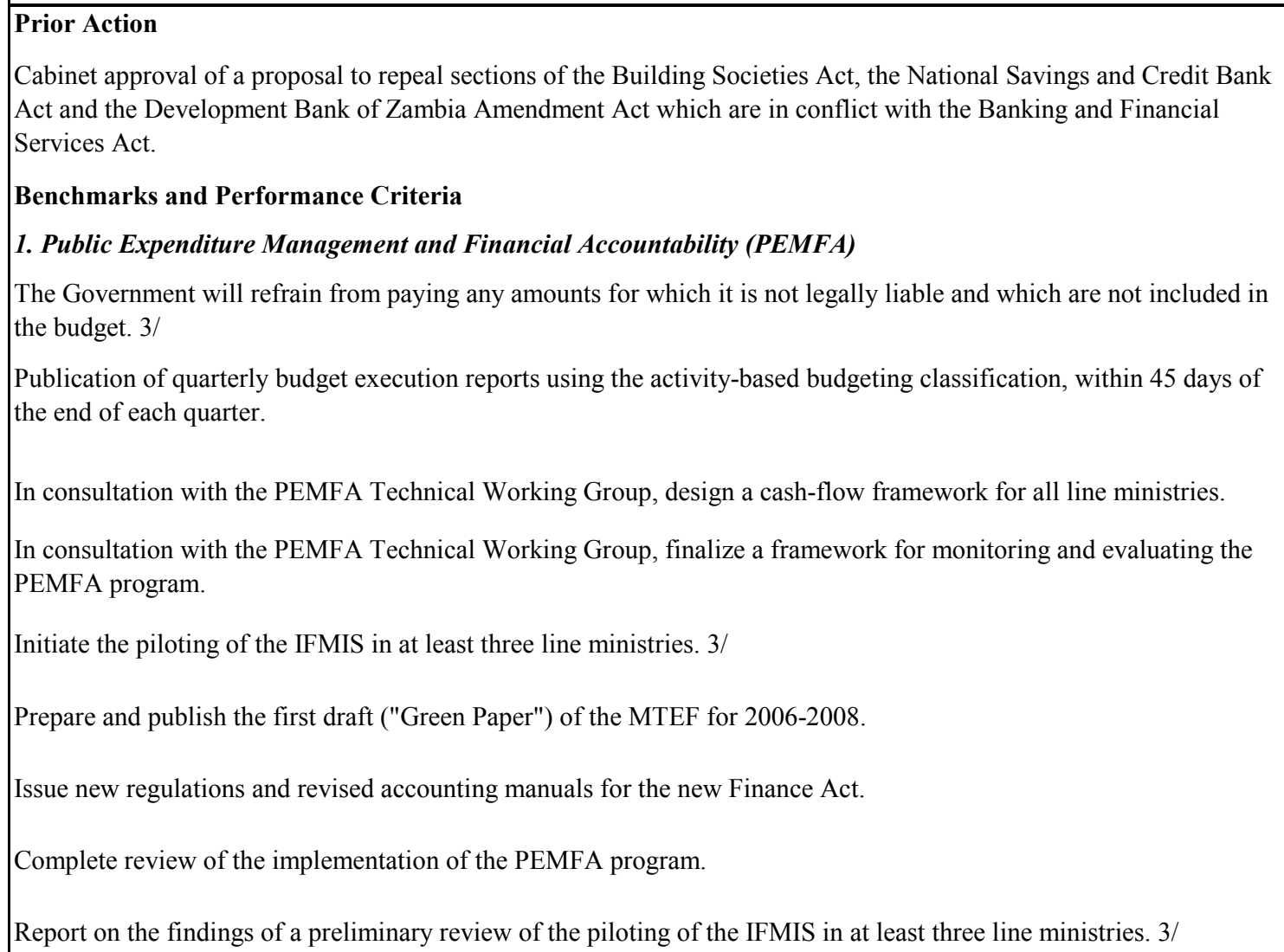

The Government will refrain from paying any amounts for which it is not legally liable and which are not included in the budget. 3/

Publication of quarterly budget execution reports using the activity-based budgeting classification, within 45 days of the end of each quarter.

In consultation with the PEMFA Technical Working Group, design a cash-flow framework for all line ministries.

In consultation with the PEMFA Technical Working Group, finalize a framework for monitoring and evaluating the PEMFA program.

Initiate the piloting of the IFMIS in at least three line ministries. 3/

Prepare and publish the first draft ("Green Paper") of the MTEF for 2006-2008.

Issue new regulations and revised accounting manuals for the new Finance Act.

Complete review of the implementation of the PEMFA program.

Report on the findings of a preliminary review of the piloting of the IFMIS in at least three line ministries. 3/

\section{Debt Management}

Beginning with the second quarter of 2005 , validate end-quarter external debt stock data and, within 45 days, provide updated 3-year schedule of debt service falling due. 3/

\section{Financial Sector Reform}

Adoption by the Government and the Bank of Zambia of an action plan, finalized in consultation with the World Bank and IMF staff, for the resolution of the Zambia National Building Society.

The NSCB and the DBZ submit to the BoZ plans for their incorporation in 2006 under the Companies Act.

\section{Governance and Transparency}

Unbudgeted expenditure requirements will be funded only to the limits of the contingency resources indicated in the budget or only after the Cabinet has approved any changes by finding compensatory funding within the approved budget resources.

1/ The definitions of the prior actions, structural benchmarks, and performance criteria are contained in the Technical Memorandum of Understanding (TMU).

2/ The prior action for the completion of the review should be carried out and reported at least five working days prior to the Executive Board discussion.

3/ Performance criteria.
Timing

Continuous

Continuous

May 2005

May 2005

June 2005

Aug 2005

Sep 2005

Nov 2005

Dec 2005

Continuous from Aug 2005

Apr 2005

Dec 2005

Continuous 


\section{Zambia: Technical Memorandum of Understanding for The Poverty Reduction and Growth Facility (PRGF) Arrangement}

\section{INTRODUCTION}

1. This memorandum sets out the understandings between the Zambian authorities and the International Monetary Fund (IMF) regarding the definitions of the quantitative and structural performance criteria and benchmarks for the program supported by the PRGF arrangement, as well as the related reporting requirements. The definitions are valid at the start of the program, but may need to be revisited to ensure that the memorandum continues to reflect the best understanding of the Zambian authorities and IMF staff to monitor the program.

\section{PRIOR ACTION}

2. The prior action described below should be carried out and reported at least five working days before the Fund's Executive Board discussion. The implementation of the prior action shall be conveyed through a written communication from the Minister of Finance and National Planning indicating that the prior actions have been observed, using the language of the prior action itself.

\section{A. Nonbank Financial Institutions}

3. Cabinet approval of a proposal to repeal sections of the Building Societies Act, the National Savings and Credit Bank Act, and the Development Bank of Zambia Amendment Act which are in conflict with the Banking and Financial Services Act.

\section{QUANTITATIVE PERFORMANCE CRITERIA AND BENCHMARKS: DEFINITIONS AND DATA SOURCES}

\section{A. Net Domestic Assets (NDA) of the BoZ}

4. Net domestic assets (NDA) of the BoZ are defined as the monthly average (based on daily data) of reserve money less net foreign assets calculated at Kwacha 4,771.3 per U.S. dollar (program exchange rate). ${ }^{1}$ Reserve money consists of currency issued, required reserves on Kwacha deposits, required reserves on foreign currency deposits (at the program exchange rate), positive current account balances of banks with the BoZ, and deposits in the $\mathrm{BoZ}$ of non-central government institutions. Net foreign assets of the BoZ are defined as gross international reserves (defined below) plus any other foreign assets, including the

\footnotetext{
${ }^{1}$ Unless otherwise defined, program exchange rates for 2005 between the U.S. dollar and other (nonKwacha) currencies will be equal to the end-December 2004 rates. Consequently, the U.S. dollar/SDR exchange rate is set at 1.5478. Any other assets (e.g. gold) would be revalued at their endDecember 2004 market prices.
} 
US\$25 million blocked reserves at the former Meridien Bank (MBZ), minus foreign liabilities (defined below). The Kwacha values are derived from the U.S. dollar values using the program exchange rate.

5. Foreign reserve liabilities are defined as short term (one year or less in original maturity) foreign currency-denominated liabilities of the $\mathrm{BoZ}$ to nonresidents and outstanding use of IMF credit. $^{2}$

6. The ceilings on NDA will be adjusted upward by the amount of the shortfall of balance of payments support net of debt service as indicated in Table 3 (item 10) of the Memorandum of Economic and Financial Policies (MEFP), up to a maximum of US\$20 million for the period end-December 2004 to end-December 2005. External disbursements that occur anytime during the month of the test date will be treated as if they were disbursed on the first day of the month. ${ }^{3}$ In the event of excess balance of payments support net of debt service, the ceiling on NDA will be adjusted downward by 100 percent of the additional excess support. However, if part of the excess support is used to reduce the stock of Treasury bills or bonds held by commercial banks and the non-bank sector, then the programmed NDA will be adjusted upward by that amount. The Kwacha value of the cumulative shortfall/excess will be calculated at the program exchange rate.

7. The ceiling on NDA will be adjusted upward up to a maximum of Kwacha 50 billion, to reflect decreases of the balance at December 31, 2004 of "Group 8 and X" accounts in the Bank of Zambia. ${ }^{4}$ The BoZ Economics Department will provide weekly data on the total balance of "Group 8 and X" accounts.

8. The ceiling on NDA will be adjusted downward/upward to reflect decreases/increases in the legal reserve requirements on deposits in commercial banks. The adjustor will be calculated as the percent change in the reserve requirement multiplied by the actual amount of reserves (Kwacha and foreign-currency denominated) at the end of the previous calendar month.

\footnotetext{
${ }^{2}$ The liability to Camdex will continue to be treated as a short-term foreign liability of the BoZ.

${ }^{3}$ This implies that, for purposes of monitoring the NDA, the targeted NDA will be increased by the amount of the disbursement divided by the number of business days in the month and multiplied by the number of business days from the beginning of the month to the day prior to the disbursement.

${ }^{4}$ The total balance at December 31, 2004 of "Group 8 and X" accounts was Kwacha 505 billion.
} 


\section{B. Net Domestic Financing (NDF)}

9. Net domestic financing is defined as the Central Government's net borrowing from the banking and nonbanking sectors (Table 1). ${ }^{5}$ All government-issued securities will be recorded at cost (face value less discount). NDF will be defined as:

(a) the net position of the Government with commercial banks, including: (i) Treasury bills; (ii) government bonds; (iii) loans and advances; less (iv) support to MBZ; and (v) central government deposits (defined to include account balances under the authority of controlling officers); plus

(b) BoZ holdings of: (i) Treasury bills; (ii) government bonds; (iii) the Kwacha bridge loan (overdraft facility); less (iv) the government's position at the BoZ; and (v) the donor suspense account; plus (vi) the long-term non-transferable security issued against the government's total indebtedness to BoZ as at end-2002.

(c) Nonbank holdings will include: Treasury bills; and government bonds.

10. The NDF ceiling will be adjusted upward by the amount of the shortfall in balance of payments support net of debt service as indicated in Table 3 (item 10) of the MEFP, up to a maximum of US\$20 million for the period end-December 2004 to endDecember 2005. In the event of excess balance of payments support net of debt service, the ceiling on NDF will be adjusted downward by 100 percent of the additional excess support. The Kwacha value of the cumulative shortfall/excess will be converted at the program exchange rate.

11. The ceiling on NDF will be adjusted upward/downward, up to a maximum of Kwacha 100 billion, to reflect decreases/increases of the balance at December 31, 2004 of "Group 8 and X" accounts in the Bank of Zambia and governments accounts in commercial banks. ${ }^{6}$ The calculation of the adjustor nets out any shortfalls/excess in domestic revenues with respect to the amounts indicated in the 2005 budget approved by Parliament.

12. The data source for the above will be the "Net Domestic Financing" Table produced by the BoZ Economics Department, submitted on a weekly basis, and reconciled with the monthly monetary survey.

\footnotetext{
${ }^{5}$ The Central Government includes all the administrations identified by the budget heads listed in the 2005 Yellow Book.

${ }^{6}$ The total balance at December 31, 2004 of "Group 8 and X" accounts was Kwacha 505.1 billion, while the total balance of government accounts in commercial banks was Kwacha 445.7 billion.
} 


\section{Gross International Reserves of the BOZ}

13. Unless otherwise noted here, gross international reserves of the BoZ will be defined as reserve assets of the BoZ (Table 2). Reserve assets are defined in the IMF BOP manual ( $5^{\text {th }}$ edition) and elaborated in the reserve template of the IMF's special data dissemination standards (SDDS). They exclude, for example, foreign assets not readily available to or controlled by the monetary authorities, and foreign currency claims on Zambian residents.

14. Gross international reserves consist of (i) monetary gold; (ii) foreign currency in cash; (iii) unencumbered foreign-currency deposits at non-resident banks; (iv) foreign securities and deposits; (v) SDR holdings and Zambia's reserve position with the IMF; and (vi) balances in the BIS account related to debt service to Paris Club creditors. Gross reserves exclude non-convertible currencies, pledged, swapped, or any encumbered reserve assets including but not limited to reserve assets used as collateral or guarantees for third-party external liabilities, commercial bank reserve requirements in foreign currency, and the US\$25 million deposit in MBZ (in liquidation).

15. The floor on gross international reserves will be adjusted: (i) downward by the amount in U.S. dollars of the shortfall in balance of payments support net of debt service as indicated in Table 3 (item 10) of the MEFP, up to a maximum of US\$20 million for the period end-December 2004 to end-December 2005; (ii) upward by 100 percent of the cumulative excess balance of payments support net of debt service. However, if part of the excess support is used to reduce the stock of Treasury bills or bonds held by commercial banks or the nonbank sector, then the programmed reserves buildup will be adjusted downward by that amount; (iii) downward/upward for any shortfall/excess in the U.S. dollar value of disbursements from the IMF under the PRGF arrangement; and (iv) downward for any increase in BoZ short-term foreign currency denominated debt (to residents and nonresidents), using the definition of short-term debt below.

16. For the purpose of this target, as well as those for external debt and arrears, valuation will be in U.S. dollars using the program exchange rate.

17. Data on gross international reserves, including its components, will be reported by the BoZ on a weekly and end-month basis.

\section{External Payment Arrears}

18. The performance criterion on the non-accumulation of new external arrears is continuous. Official external payment arrears are defined as unpaid debt service by the Central Government and BoZ, beyond the due date and/or the grace period, if any. This definition excludes arrears subject to rescheduling. It also excludes the accumulation of external arrears that arise from the transfer to non-residents of foreign currency denominated obligations owed by the Government of Zambia to resident creditors. 
19. Data on arrears are compiled jointly by the Ministry of Finance and National Planning (MoFNP) and BoZ and will be reported by the MoFNP on a quarterly basis.

\section{E. Official Medium- and Long-Term Concessional External Debt}

20. Official medium- and long-term concessional external debt is defined as all forms of official debt with original maturity of more than one year contracted or guaranteed by the Central Government and BoZ having a grant element of more than 40 percent, but excludes debts subject to rescheduling. The grant element is to be calculated by using currencyspecific commercial interest reference rates (CIRRs) reported by the OECD; for maturities of less than 15 years, the grant element will be calculated based on six-month averages of CIRRs, and for maturities longer than 15 years, the grant element will be calculated based on 10 -year averages. Adjustment lending from the IMF will be excluded.

21. This target applies not only to debt as defined in Point 9 of the Guidelines on Performance Criteria with Respect to Foreign Debt (Decision No. 12274-(00/85) of August 24, 2000; see Annex), but also to commitments contracted or guaranteed for which value has not been received. This excludes non-concessional loans stemming from the rescheduling of external arrears.

22. Detailed data on all new concessional and non-concessional debt contracted or guaranteed will be provided by the MoFNP on a monthly basis.

\section{F. Official External Short-Term Non-concessional External Debt}

23. Official external short-term non-concessional external debt is defined as the outstanding stock of external debt with original maturity of less than one year owed or guaranteed by the central Government or the BoZ. For this purpose short-term debt will include forward commodity sales but will exclude normal trade credit for imports. There will be no new official external short-term debt during the program period. The term "debt" has the meaning set forth in point No. 9 of the Guidelines on Performance Criteria with Respect to Foreign Debt (Decision No. 12274-(00/85) of August 24, 2000; see Annex).

24. The data will be reported by the MoFNP and BoZ on a monthly basis.

\section{G. Collateralizing/Guaranteeing of Loans to the Zambia Electricity Supply Corporation (ZESCO)}

25. The Government and the BoZ shall not extend or guarantee any new commercial debts of the Zambia Electricity Supply Corporation (ZESCO), including in the form of loans, suppliers credits and leases. New concessional borrowing, as defined above (Paragraph 20), will be subject to a cumulative limit of US\$20 million in 2005. The limit on new concessional borrowing will be subject to review, in consultation with IMF and World Bank staff, in the event that additional concessional resources are required. 


\section{H. Domestic Arrears of Government}

26. Domestic arrears are defined as: (i) any bill that has been received by a spending ministry from a supplier for goods and services delivered (and verified) and for which payment has not been made within 30 days after the due date of payments; (ii) wage, salary and any payment to government employees, including all forms of housing allowances, that were due to be paid in a given month but remained unpaid on the $15^{\text {th }}$ of the following month; and (iii) interest or principal obligations which remain unpaid 30 days after the due date of payment.

27. Information regarding domestic arrears is to be compiled through audits of the accounts of spending Ministries and agencies, conducted by the Internal Audit division of the MoFNP. The audits will be completed and data submitted to IMF staff by the Secretary of the Treasury within six weeks of the end of each quarter.

\section{The Central Government's Wage Bill}

28. For the purposes of the wage bill, the definition of Central Government includes all heads covered in the 2005 Yellow Book. The Central Government's total wage bill will include payments on wages, salaries, allowances, and all other items specified as personal emoluments in the Yellow Book, and any direct or indirect payments of housing allowances to employees. The Government will provide, on a monthly basis and by budget head, the following data: (i) the number of all employees in the Central Government for each budget head; (ii) the basic salary, the allowances and any other personal emoluments released during the month; (iii) the arrears incurred during the month on the basic salary, on the allowances, and on any other personal emoluments; (iv) the number of employees retrenched and the corresponding retrenchment costs.

29. All the data will be submitted to the IMF staff by the MoFNP within three weeks of the end of each month.

\section{STRUCTURAL PERFORMANCE CRITERIA AND BENCHMARKS}

\section{A. Public Expenditure Management}

30. The Government will refrain from making payments for which it is not legally liable and which are not included in the budget. It will also guide the liquidation and receivership process of public enterprises to minimize the Government's liabilities consistent with the law.

31. The MoFNP will publish quarterly budget execution reports using the classification system of activity-based budgeting within 45 days of the end of each quarter.

32. The MoFNP, in consultation with the Public Expenditure Management and Financial Accountability (PEMFA) program Technical Working Group, will design a cash-flow 
framework for all line ministries. The Accountant General will notify, by June 15, 2005, the IMF Resident Representative's Office that the cash-flow framework's design has been completed.

33. The MoFNP, in consultation with the Public Expenditure Management and Financial Accountability (PEMFA) program Technical Working Group, will develop a framework for monitoring and evaluating the PEMFA program. The Accountant General will submit, by June 15, 2005, to the IMF Resident Representative's Office the final framework for monitoring and evaluating the PEMFA program.

34. The Government will initiate the piloting of the Integrated Financial Management and Information System (IFMIS) in at least three ministries as a key step to introduce an effective system in all ministries and provinces. The Accountant General will notify, by July 15, 2005, the IMF Resident Representative's Office the initiation of the piloting.

35. The Government will prepare and publish, by end-August 2005, a first draft of the Medium-Term Expenditure Framework (“Green Paper”) for the period 2006-2008.

36. The MoFNP will issue the new regulations and the revised accounting manual needed for the complete and effective operations of the Finance Act approved by parliament in December 2004. The MoFNP will notify, by October 15, 2005, the IMF Resident Representative's Office the issuance of the statutory instrument needed to put in place the new regulations and the publication of the revised accounting manual.

37. On the basis of the monitoring and evaluating framework defined in consultation with the PEMFA Technical Working Group, the MoFNP will complete the review of the implementation of the PEMFA program by November 2005. The review will provide information on progress achieved, possible shortcomings, and specific measures to address weaknesses.

38. By December 2005, the Government will report the findings of a preliminary review of the piloting of the IFMIS in at least three ministries. This preliminary review will lead to the completion of a mid-term review of the pilot program as envisaged in the Enhanced HIPC Initiative completion point document.

\section{B. Debt Management}

39. Beginning with the second quarter of 2005, the MoFNP will validate end-quarter external debt stock data and, within 45 days, provide updated 3-year schedule of debt service falling due. 


\section{Financial Sector Reform}

40. Adoption by the Government and the Bank of Zambia of an action plan, finalized in consultation with the World Bank and IMF staff, for the resolution of the Zambia National Building Society.

41. The National Savings and Credit Bank and the Development Bank of Zambia will submit, by end-December 2005, to the BoZ plans for their incorporation in 2006 under the Companies Act.

42. The Zambia Privatization Agency will, by end-May, issue a new invitation for bids for the purchase of 49 percent equity in the Zambia National Commercial Bank with management control. The MoFNP will confirm in writing to Fund staff that this step has been taken, attaching a copy of the invitation for bids.

\section{Governance and Transparency}

43. Unbudgeted expenditure requirements will be funded only to the limits of the contingency resources indicated in the budget or only after the Cabinet has approved any changes by finding compensatory funding within the approved budget resources. 


\title{
ANNEX \\ Guidelines on Performance Criteria with Respect to Foreign Debt
}

\author{
Excerpt from Executive Board Decision No. 6230-(79/140), as revised on August 24, 2000
}

9. (a) For the purpose of this guideline, the term "debt" will be understood to mean a current, i.e., not contingent, liability, created under a contractual arrangement through the provision of value in the form of assets (including currency) or services, and which requires the obligor to make one or more payments in the form of assets (including currency) or services, at some future point(s) in time; these payments will discharge the principal and/or interest liabilities incurred under the contract. Debts can take a number of forms, the primary ones being as follows:

(i) loans, i.e., advances of money to the obligor by the lender made on the basis of an undertaking that the obligor will repay the funds in the future (including deposits, bonds, debentures, commercial loans and buyers' credits) and temporary exchanges of assets that are equivalent to fully collateralized loans under which the obligor is required to repay the funds, and usually pay interest, by repurchasing the collateral from the buyer in the future (such as repurchase agreements and official swap arrangements);

(ii) suppliers' credits, i.e., contracts where the supplier permits the obligor to defer payments until some time after the date on which the goods are delivered or services are provided; and

(iii) leases, i.e., arrangements under which property is provided which the lessee has the right to use for one or more specified period(s) of time that are usually shorter than the total expected service life of the property, while the retains the title to the property. For the purpose of the guideline, the debt is the present value (at the inception of the lease) of all lease payments expected to be made during the period of the agreement excluding those payments that cover the operation, repair or maintenance of the property.

(b) Under the definition of debt set out in point 9 (a) above, arrears, penalties, and judicially awarded damages arising from the failure to make payment under a contractual obligation that constitutes debt are debt. Failure to make payment on an obligation that is not considered debt under this definition (e.g., payment on delivery) will not give rise to debt. 
Table 1. Net Domestic Financing

\begin{tabular}{|c|c|c|c|}
\hline & 200 & & 2005 \\
\hline & End-Dec. & $\begin{array}{l}\text { End-Dec. } \\
\text { ogram Base }\end{array}$ & \\
\hline Total domestic financing (program) & $3,666,556$ & $3,666,556$ & \\
\hline Adjustment & 53,325 & 53,325 & \\
\hline Adjusted program financing & 0 & 0 & \\
\hline Excess/shortfall $(-=$ excess $)$ & 307,843 & 307,843 & \\
\hline Total domestic financing & $3,305,388$ & $3,305,388$ & \\
\hline Bank financing & $2,518,496$ & $2,518,496$ & \\
\hline Commercial banks & $1,277,128$ & $1,277,128$ & \\
\hline Treasury bills $1 /$ & 886,980 & 886,980 & \\
\hline Bonds 1/ & 841,987 & 841,987 & \\
\hline Loans and advances & 277 & 277 & \\
\hline less: Support to MBZ & $-8,423$ & $-8,423$ & \\
\hline less: Deposits & $-443,693$ & $-443,693$ & \\
\hline Bank of Zambia & $1,241,368$ & $1,241,368$ & \\
\hline Treasury bills $1 /$ & 62,581 & 62,581 & \\
\hline Bonds 1/ & 13,819 & 13,819 & \\
\hline Kwacha bridging loan & 409,629 & 409,629 & \\
\hline GRZ position & $-805,121$ & $-805,121$ & \\
\hline Donor suspense balance & $-86,284$ & $-86,284$ & \\
\hline GRZ long-term security IFO BoZ & $1,646,743$ & $1,646,743$ & \\
\hline Other & 0 & 0 & \\
\hline Nonbank financing & 786,892 & 786,892 & \\
\hline Treasury bills $1 /$ & 391,183 & 391,183 & \\
\hline Bonds 1/ & 395,710 & 395,710 & \\
\hline
\end{tabular}

Source: BoZ net domestic financing table.

1/ Measured at cost (face value less discount) starting from end-December 2003. 
Table 2. Zambia: Gross International Reserves 1/

(In millions of U.S. dollars)

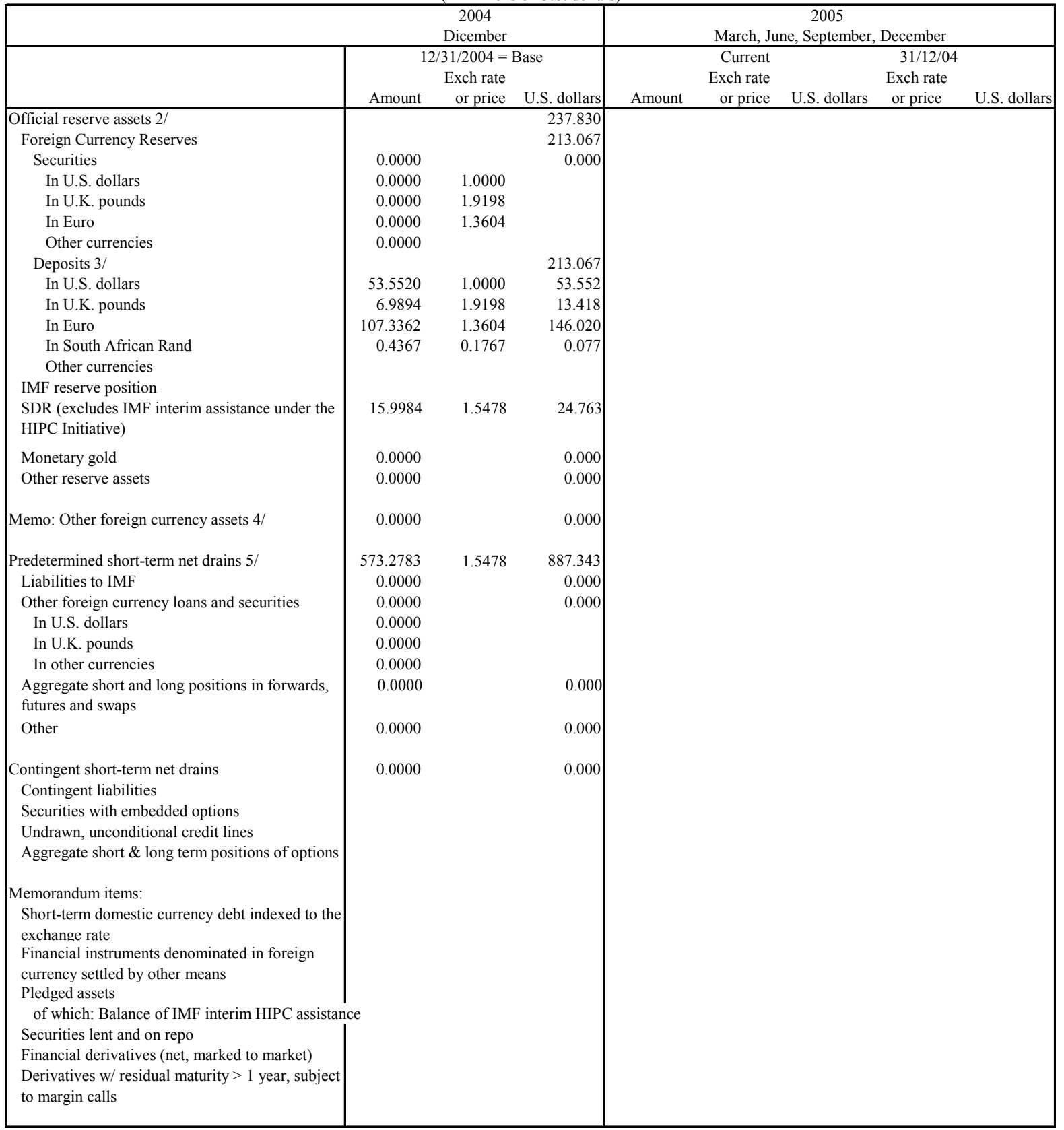

1/ As defined in the TMU or IMF, "Data Template on Int'l Reserves and Foreign Currency Liquidity: Operational Guidelines."

$2 /$ Corresponds to gross international reserves for program monitoring.

3/ Excludes deposits at resident banks, unless assets held abroad by the bank are explicitly connected to the foreign exchange deposits of the BoZ

and are totally and effectively controlled by BoZ and are available for balance of payment needs.

4/ Includes foreign currency deposits at resident banks.

5/ The program target for gross international reserves will be adjusted as described in the TMU. 
Zambia: Fund Relations

(As of January 31, 2005)

I. Membership Status: Joined: September 23, 1965; Article VIII

II. General Resources Account:

SDR Million

489.10

489.10

0.2

Reserve position in Fund

III. SDR Department:

Net cumulative allocation

Holdings

IV. Outstanding Purchases and Loans:

PRGF arrangements

SAF loans

V. Latest Financial Arrangements:

Type Approval

Date

PRGF

PRGF

PRGF
$06 / 16 / 2004$

$03 / 25 / 1999$

$12 / 06 / 1995$
Expiration

Date

$06 / 15 / 2007$

$03 / 28 / 2003$
$12 / 05 / 1998$
SDR Million

68.30

16.00

SDR Million

536.93

36.35
(SDR Million)

278.90

701.68
Percent

Quota

100.0

100.0

0.0

Percent

Allocation

100.0

23.4

Percent

Quota

109.8

7.4

VI. Projected Obligations to Fund (Without HIPC Assistance):

(SDR million; based on existing use of resources and present holdings of SDRs)

Principal

Charges/interest

Total

\begin{tabular}{rrrrr}
\multicolumn{6}{c}{ Forthcoming } \\
\hline$\frac{2005}{170.69}$ & $\underline{2006}$ & $\frac{2007}{26.95}$ & $\frac{2008}{47.50}$ & $\frac{2009}{54.76}$ \\
$\frac{3.81}{174.50}$ & $\frac{3.20}{13.69}$ & $\frac{3.12}{30.07}$ & $\frac{2.95}{50.46}$ & $\frac{2.71}{57.47}$
\end{tabular}

Amount Drawn

(SDR Million)

165.07

237.52

661.68 


\section{Implementation of HIPC Initiative:}

Enhanced

Framework

Commitment of HIPC assistance

Decision point date

Dec. 2000

Assistance committed (1999 NPV terms) ${ }^{19}$

Total assistance (US\$ million)

$2,499.00$

Of which: Fund assistance (SDR million)

Completion point date

Floating

Delivery of Fund assistance (SDR million)

Amount disbursed

Interim assistance

Completion point

Amount applied against member's obligations (cumulative) $^{21}$

351.60

\section{Safeguards Assessment:}

Under the Fund's safeguards assessment policy, the Bank of Zambia (BOZ) is subject to an assessment with respect to the PRGF arrangement, which was approved on June 16, 2004 and is scheduled to expire on June 15, 2007. A safeguards assessment of the BOZ was completed on October 20, 2004.

The assessment identified certain weaknesses. The authorities have taken the following steps in the light of the recommendations made:

(i) in December 2004, the BoZ's board adopted the International Financial Reporting Standards as the basis for financial reporting;

\footnotetext{
${ }^{19}$ Net present value (NPV) terms at the decision point under the enhanced framework.

${ }^{20}$ Including an additional grant contribution of SDR 16.4 million from the Netherlands to Zambia in the context of the enhanced HIPC Initiative.

${ }^{21}$ The amount applied against Zambia's obligations includes interest income earned on resources while in Zambia's subaccount within the Umbrella Account for HIPC Operations.
} 
(ii) in December 2004, the BoZ's board also adopted a policy for the appointment of external auditors (three year-term appointment, renewable only once);

(iii) pending the approval of an amendment to the Bank of Zambia Act the BoZ and the MoFNP are finalizing a Memorandum of Understanding indicating - for fiscal year 2004the exclusion of unrealized gains from profits available for distribution to government; and

(iv) the Finance Department of the BoZ prepared a formal format for reconciling the data reported to the Fund and, jointly with the Economics Department of the BoZ, set up an arrangement to improve and speed up reconciliation exercises.

Finally, the MoFNP is discussing measures to strengthen the operational independence of the $\mathrm{BoZ}$ that are expected to result in amendments to the Bank of Zambia Act.

\section{Exchange Rate Arrangement:}

The currency of Zambia is the kwacha. The exchange rate for the kwacha is determined in the inter-bank foreign exchange market, in a managed float with no preannounced path. On December 31, 2004, the Bank of Zambia mid-rate was K 4,771.3 per U.S. dollar. On April 19, 2002, Zambia accepted the obligations of Article VIII, Section 2, 3, and 4 of the Articles of Agreement. However, the Fund urged the authorities to eliminate the exchange restriction evidenced by the accumulation of external payments arrears, which is subject to approval by the Fund under Article VIII, as soon as possible.

\section{Article IV Consultations:}

Zambia is on the standard 12-month Article IV consultation cycle, subject to the provisions of the decision on consultation cycles approved on July 15, 2002. The last Article IV consultation was concluded by the Executive Board on April 7, 2004.

\section{FSAP Participation and ROSC:}

Zambia has participated in the financial sector assessment program (FSAP), and an FSAP mission from the Fund and the World Bank conducted a comprehensive external assessment of the financial system. The two FSAP missions visited Zambia during April 30-May 15 and July 15-26, 2002.

A fiscal transparency module of a Report on Observance of Standards and Codes (ROSC) assessing compliance with the IMF's Code of Good Practices on Fiscal TransparencyDeclaration of Principles was completed and issued to the Executive Board on October 31, 2001. A ROSC-Data Module was prepared in June 2004. 
XII. Technical Assistance (since 1997):

\section{Resident advisors}

\section{Department}

MFD

FAD
1997-2001

2002-2003

\section{Dates}

1994- 98

1998-99

1998-99

2002-2003

\section{Technical assistance missions}

Department

MFD

STA

\section{Dates}

November 2000

June 2001

November 2001

September 2002

January and May 2003

September 2003

April and August 2004

June 1997

November 1997

March 1998

May 2000

June 2002

June 2002

June 2002

July 2002

June 2004

\section{Position}

Senior Operations Advisor

Monetary Advisor on Monetary Policies and Investments

Advisor on Monetary Operations

Advisor on Budget Management

Advisor on Public Expenditure

Management

\section{Purpose}

Bank supervision

Bank supervision

Monetary policy, financial sector issues, and payments system

Foreign exchange market

Development of foreign exchange

interbank market, monetary operations, and reform of the financial system

Monetary operations, reform of the financial system, government securities market, and payments system.

Resolution of nonbank financial institutions

Monetary survey (combined STA/AFR mission)

Balance of payments

Monetary survey

Balance of payments

General Data Dissemination System

(GDDS)

Government Finance Statistics (GDDS)

Real Sector (GDDS)

Monetary Statistics (GDDS)

Data ROSC 
FAD

LEG
March 2000

January 2001

May-June, 2001

August 2001

December 2002
Budget management

Public expenditure management

ROSC - fiscal transparency module

Tax policy issues

Inspection mission to review the progress

in public expenditure management reform

August-December 2004 Assistance on amending legislation on nonbank financial institutions

\section{Resident Representative:}

A Fund Resident Representative first took up the position in Lusaka in June 1990. Mr. Joseph Kakoza has been the Resident Representative since October 2003. 


\section{Zambia: Relations with the World Bank Group}

\section{A. Partnership for Development}

1. The IDA and IMF Boards endorsed the Government's Poverty Reduction Strategy, covering the period 2002-2004, embodied in its first full PRSP, in May of 2002. An Interim Poverty Reduction Strategy Paper (I-PRSP) had been presented to the Board in July 2000. The PRSP was substantially complete when the new Government took office in January 2002. The new Government endorsed the PRSP and went further to expand the PRSP in the Transitional National Development Plan (TNDP) that was published in October 2002 and covers the period 2002-2005. The first and the second Annual Progress Report on the implementation of the PRSP were presented to the IDA and IMF Boards in May 2004 and March 2005, respectively.

2. Strategic focus of the PRSP. The PRSP /TNDPhighlights the following barriers to poverty reduction: lack of economic growth, high inequality, excessive dependence on foreign aid, poor prioritization of public resources, inadequate safety nets, and HIV/AIDS. Therefore, the PRSP seeks to achieve poverty reduction through promoting (i) economic growth and diversification of production and exports; (ii) improved access and quality in the provision of social services; and (iii) the mainstreaming of crosscutting issues of governance, HIV/AIDS, gender, and the environment. Targets and indicators are provided for each sector and sub-sector. For example, by 2004 the poverty headcount is to be reduced to 65 percent, the same as in 1996. Even though the MDGs are not explicitly mentioned in the PRSP, given that the MDGs were not widely discussed when Zambia prepared its PRSP, some of the targets for improvements in the social indicators are similar to the MDGs.

3. Joint staff assessment of the PRSP and the Progress Reports. Bank and IMF staffs are in agreement with the major objectives set out in the PRSP and confirmed in the Annual Progress Reports. The strengths of the PRSP are: a participatory process that involved civil society, the private sector, and the donors; a poverty analysis that draws from available quantitative and qualitative data, looks at multiple dimensions of poverty, and provides data broken down by socio-economic group, gender, and region; an appropriate macroeconomic framework and financing plan; identification of priority sectors likely to contribute most to pro-poor growth; and the emphasis placed on the need to improve governance and reduce corruption.

4. Since 1995, the Fund has supported by ESAF and PRGF arrangements Zambia's economic reform program. During December 2003-May 2004, the authorities have implemented an extended Staff Monitoring Program (SMP), which has formed the basis of a new Fund-supported program under the Poverty Reduction and Growth Facility which was endorsed by the IMF Board on June 14, 2004. The PRGF focuses on (i) fiscal consolidation; (ii) inflation reduction, and (iii) maintaining an adequate level of foreign reserves. Successful 
performance under the PRGF is a key condition for reaching the completion point under the Enhanced HIPC Initiative.

\section{B. World Bank Group Strategy}

5. In March 2004, the Bank's executive Board discussed the Bank's current Country Assistance Strategy (CAS), which outlines the roadmap for the Bank's country support for the period 2004-2007. The CAS is explicitly rooted in a result-based framework, which links overall goals of the PRSP/TNDP to specific strategic objectives. Paying attention to causeand-result linkages, the main focus of the planned Bank's activities is to remove obstacles to longer-term strategic development outcomes. Where appropriate, quantitative targets to be reached during the CAS period have been specified. For the CAS period, the Bank will track progress on specific outcomes and intermediate indicators directly affected by Bank interventions.

6. World Bank activities focus on three strategic priorities:

1. Strategic Priority 1: Sustained Economic Growth Anchored in a Diversified and Export-Oriented Economy;

2. $\quad$ Strategic Priority 2: Improved Lives and Protection of the Vulnerable;

3. Strategic Priority 3: Efficiently and Effectively Managed Public Sector.

In addition to ongoing Bank's lending operations in the areas of public sector reform, privatization, HIV/AIDS, education, social service delivery, infrastructure, support for diversification, and the environment, the base case of the new CAS includes adjustment lending, i.e., the Economic Management and Growth Credit, approved in December 2004. the new CAS also continues project support (in particular, for public sector reforms, infrastructure, and sanitation) and pursues a reengagement in the agricultural sector.

7. The Bank continues to undertake substantive country diagnostics providing a solid base for policy dialogue, design, and implementation of its lending operations in Zambia. A combined Public Expenditure Review (PER), Country Procurement Assessment Review (CPAR), and Country Financial Accountability Assessment (CFAA), was completed in 2003 and forms the basis for the Public Expenditure and Financial Accountability TA work jointly undertaken with cooperating partners. The main economic sector works recently completed are a Country Economic Memorandum (CEM), an Investment Climate Assessment (ICA), an Administrative Barriers study (AB), and a Strategic Country Gender Assessment (SCGA). In addition, a collaborative Poverty and Vulnerability Assessment (PVA) and Public Expenditure Review for the education sector are currently underway.

8. For FY06/07 a review of labor market regulations, a rural development strategy, a post privatization copper strategy, and an integrated framework study for trade have been planned. In the social sectors, the Bank plans to undertake a public expenditure review of the health sector and a review of utility regulations and tariff setting mechanisms in FY07. 
9. Zambia had concluded the 1999-2003 CAS in the low case scenario, which had become the departure platform for the current CAS. Starting from the low case of the last CAS of about US\$65 million in IDA commitments per year (actual for FY02/03 were US\$132 million in total) the proposed resource envelope under the new low case scenario is US\$180-240 million for the period FY04-07. Currently Zambia is in the base case scenario, which results in US\$70-80 million p.a., or between US\$280-320 million in total. This amount implies an exhaustion of the current IDA allocation (SDR230 million for FY04-07). In case Zambia's performance is sustained or accelerate with regard to macroeconomic and structural reforms and PRSP implementation, the new high case would call for exceptional allocation of IDA resources during the 2004-2007 CAS, i.e., between US\$90-105 million p.a. or US\$360-420 million in total (an increase by 30 percent over the current IDA envelope).

10. The Bank's active portfolio as of February 2005 contains 17 IDA credits for a total commitment of US\$677.1 million (see Table 1). During FY04, one project was approved, while 3 projects were approved during FY05: (i) Road Rehabilitation and Maintenance project in March 2004; (ii) GEF SEED Biodiversity, in July 2004; (iii) SEED LiL to support economic diversification in July 2004; and (iv) Economic management and Growth Credit in December 2004.

Table 1: Projects under Supervision

(As of $02 / 06 / 05$ )

\begin{tabular}{|c|c|c|c|c|c|c|}
\hline 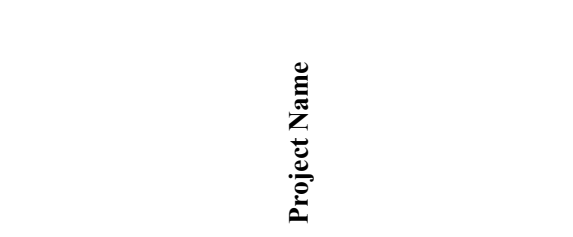 & 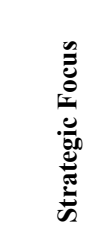 & 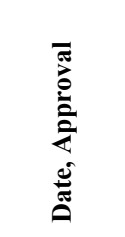 & 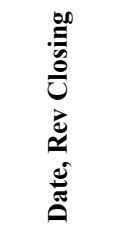 & 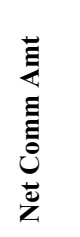 & $\begin{array}{l}\stackrel{0}{0} \\
\stackrel{0}{0} \\
\stackrel{0}{0}\end{array}$ & 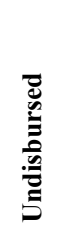 \\
\hline Totals & & & & 677.1 & 441.6 & 235.5 \\
\hline ZM-Econ Mgmt \& Growth SAC (FY05) & Pillar 1 & $12 / 14 / 04$ & $12 / 31 / 05$ & 40.0 & 20.0 & 20.0 \\
\hline ZM-SEED SIL (FY05) & Pillar 1 & $7 / 29 / 04$ & $11 / 30 / 09$ & 28.2 & 2.4 & 25.7 \\
\hline ZM-GEF SEED Biodiversity SIL (FY05) & Pillar 2 & $7 / 29 / 04$ & $5 / 31 / 09$ & 4.0 & 0.3 & 3.8 \\
\hline ZM-Road Rehab Maintenance Prj (FY04) & Pillar 1 & $3 / 9 / 04$ & $6 / 30 / 07$ & 50.0 & 3.0 & 47.0 \\
\hline Copperbelt Environment & Pillar 1 & $3 / 20 / 03$ & $8 / 1 / 08$ & 40.0 & 3.3 & 36.7 \\
\hline HIV/AIDS (ZANARA) & Pillar 2 & $12 / 30 / 02$ & $2 / 28 / 08$ & 42.0 & 9.4 & 32.6 \\
\hline ZA:Emergency Drought Recovery Project & Pillar 1 & $11 / 19 / 02$ & $6 / 30 / 05$ & 50.0 & 46.1 & 3.9 \\
\hline ZM-TEVET SIM (FY01) & Pillar 2 & $6 / 14 / 01$ & $12 / 30 / 06$ & 25.0 & 9.2 & 15.8 \\
\hline Regional Trade Fac. Proj. - Zambia & Pillar 1 & $4 / 3 / 01$ & $6 / 30 / 11$ & 15.0 & 7.7 & 7.3 \\
\hline ZM RAILWAYS RESTRUCTURING PROJECT & Pillar 1 & $11 / 16 / 00$ & $6 / 30 / 05$ & 27.0 & 27.8 & -0.8 \\
\hline MINE TOWNSHIP SERVICES PROJECT & Pillar 2 & $6 / 20 / 00$ & $12 / 31 / 05$ & 37.7 & 25.8 & 11.9 \\
\hline Social Investment Fund (ZAMSIF) & Pillar 2 & $5 / 25 / 00$ & $12 / 31 / 05$ & 64.7 & 60.7 & 4.0 \\
\hline PUB SVC CAP (PSCAP) & Pillar 3 & $3 / 28 / 00$ & $6 / 30 / 05$ & 28.0 & 26.9 & 1.1 \\
\hline Basic Ed. Subsect. Inv. Pgr & Pillar 3 & $4 / 8 / 99$ & $6 / 30 / 05$ & 40.0 & 27.6 & 12.4 \\
\hline ZM POWER REHABILITATION & Pillar 1 & $2 / 19 / 98$ & $12 / 31 / 05$ & 75.0 & 68.2 & 6.8 \\
\hline ZM NATIONAL ROAD & Pillar 1 & $10 / 14 / 97$ & $3 / 31 / 05$ & 70.0 & 69.0 & 1.0 \\
\hline ERIPTA & Pillar 1 & $6 / 4 / 96$ & $9 / 30 / 05$ & 40.5 & 34.1 & 6.4 \\
\hline
\end{tabular}


11. The International Finance Corporation (IFC) is very much aligned with the Bank's policy agenda in Zambia. The IFC currently has a small portfolio of 12 projects totaling US\$15 million outstanding. These are a mix of financial sector, agribusiness, telecommunications (mobile telephone), and tourism projects. The IFC's most prominent project was for US\$30 million in the equity of Konkola Copper Mines (KCM) with AngloAmerican in 2000. The IFC exited in 2002 along with Anglo, in accordance with the terms of the investment agreement.

12. Zambia is currently a group 3 country for the IFC (as defined in the recently board approved IFC strategic initiative for Africa) and thus assessed as a relatively poor investment climate, with limited prospects for stimulating new private investment and limited progress on reforms to improve the investment climate. In this environment, the IFC seeks to be responsive to individual projects where IFC can play a role, and in which there is strong development impact, but does not invest significant resources in upstream project development. The IFC is exploring, jointly with the Bank, how to support the Bank Group's activities on improving the investment climate in Zambia.

13. MIGA's outstanding portfolio in Zambia consists of four contracts of guarantee in the agribusiness and manufacturing services sector with a US\$36.1 million gross exposure and a US\$35.7 million net exposure as of December 2003. The projects are for the privatization, modernization, and expansion of a foundry, and for cobalt and copper facilities. They benefit the Zambian economy by saving production costs and by providing training to personnel working on projects. The estimated total amount of foreign direct investment facilitated to date is US\$155.5 million

\section{IMF-World Bank Collaboration in Specific Areas}

14. A summary of IMF-World Bank collaboration in Zambia is provided in Table 2 . There are a number of areas where the Fund leads and its analysis serve as inputs into the World Bank policy formulation and advice, including policies to maintain macroeconomic stability, fiscal, monetary, and exchange rate policies. There are other areas in which the Fund and the Bank share responsibility and are coordinating closely their policy advice to the Zambian authorities, such as trade, financial sector, public expenditure management, including debt management, and economic governance. The Bank has taken the lead in the social sectors, including health, education, social protection, water and sanitation, agriculture and rural development, private sector development including regulatory issues and the environment.

15. The Fund and the Bank shared joint responsibility in supporting the Government in the preparation of the PRSP and its annual progress reports on the implementation of the PRSP. The Fund and the Bank have also jointly provided assistance to Zambia for evaluating progress to reach the Completion Point under the Enhanced HIPC Initiative. In addition, the Bank and the Fund will jointly evaluate Zambia's debt and cash management practices. 


\section{C.1. Areas in which the World Bank leads and there is no direct IMF involvement}

16. Areas in which the Bank leads and there is no direct involvement of the IMF are support for social sectors, agriculture and rural development, economic diversification, infrastructure, and the environment. In light of the complex and crosscutting nature of risk and vulnerability in Zambia, the Bank is assisting Government in developing a cross-sectoral Social Protection Strategy. The strategy will extend beyond traditional ex post risk-coping measures (e.g. safety nets) to include a better understanding of the role of ex ante riskprevention and risk-mitigation measures. The strategy is being prepared in a highly participatory fashion, with the aim of integrating it within Zambia's next poverty-reduction strategy. For the same purpose, the Bank is in the process of undertake a Poverty and Vulnerability Assessment, which is to be discussed with GRZ in spring 2005.

17. HIV/AIDS and health. At this time, IDA is assisting the Ministry of Health with a US\$12 million project under a multi-sectoral AIDS program (ZANARA). In addition, IDA has carried out an ESW on human resources in the health sector (FY04) and plans a health sector PER in FY06. Building on this, IDA will provide support, but not necessarily financially for the Health Sector Support Program (SWAP) beginning in FY07. The improvement of water supplies under the Mine Township Services Project will also contribute to health outcomes in the areas served.

18. In education, the Bank is currently supporting the Ministry of Education's efforts through Adaptable Program Lending (APL). The APL1 Credit enables IDA funds to be devoted to a wide range of purposes in basic education and, following an amendment, to construct two high schools in the Eastern Province. The Bank is supporting expenditure effectiveness and efficiency in the sector through a public expenditure review (currently underway).

19. In the area of technical education, vocational, and entrepreneurship training (TEVET), the Government is carrying out reforms aimed at improving the quality of training, making it more responsive to labor market demand and financially sustainable. Reforms also aim at increasing the participation of female trainees and trainees from socio-economically disadvantaged groups. The Government's efforts in this area are being supported by an IDA Credit committed in FY01 and by several other partners.

20. In agriculture and rural development, the Bank's focus is two fold: improvement of productivity of the sector and increase of overall food security. The Bank is reengaging in the agricultural sector given the priority Zambia attaches to this sector, as set out in the PRSP/TNDP. Indeed, in a stable macroeconomic environment, if supporting infrastructure and adequate agricultural policy are in place, a major effort could be launched to unleash growth and poverty reduction potential of the sector, in particular through well-targeted interventions for both smallholders and commercial agriculture.

21. Analytical and Advisory Activities (AAA) are planned to support the formulation of an agricultural policy consistent with the planned and ongoing projects in related sectors, 
such as roads and electricity, to remove bottlenecks that prohibit and or make difficult for farmers to produce for the market. Hence, the policy dialogue with the Government on reforms and priorities of public spending for agriculture and rural development will address both short run policy issues, as well as a long run strategy to tap the large agricultural export potential of the country, focusing on investment in land development, irrigation, and infrastructure. A balanced treatment of smallholders and commercial agriculture is important for growth and poverty reduction. As a follow-up to the AAA work, an Agricultural Support Project is planned to assist the Government with these challenges.

22. In addition, the Bank is assisting Zambia in developing an Integrated Framework for Disaster Relief and Mitigation-intended as multi-sectoral response to anticipate and address the impacts of climate shocks-under the ongoing Emergency Drought Recovery Project (EDRP). The aim is to increase food security and protect living conditions for the poorest and most vulnerable in targeted districts. Pilot programs are being designed to manage and protect against climate shocks, including dams and local irrigation, labor intensive public works, and agriculture input distribution programs. Feeding programs will be implemented in primary schools in a subset of districts; young children in Zambia are particularly at risk to high and rising levels of malnutrition.

23. The Bank supports economic diversification directly through its SEED project, which will assist implementation of a diversification strategy in the tourism, agribusiness, and gemstone sectors. In the initial phase, the SEED project will focus on supporting the design and establishment of appropriate policy and legal frameworks to encourage private investments in these three sectors, as well as investments, primarily in tourism-related infrastructure. The tourism component will work toward making Livingstone the flagship tourism destination in Zambia and strengthening management of national parks, which underpin Zambia's medium-term tourism development strategy. The interventions in the gemstones and agribusiness subsectors are focused to strengthen the supply chain that links rural/small-scale producers to local and export markets.

24. Private sector development. To improve Zambia's investment climate, the Bank and the IFC have assisted the Government with analytical work, notably an Investment Climate Assessment and a FIAS-led Administrative Barriers to Investment study. These studies are geared to facilitate the adoption of a new and more business friendly Investment Act and result in regular consultations between business and Government to discuss how to strengthen Zambia's business climate. As a result of this dialogue, the Government, with support from the Bank and the IFC, prepared a private sector development strategy. The strategy was developed through an interactive process, which included intensive consultations with the private sector and other donors and coordination within the Government itself. Implementation of some of the reform actions identified in the strategy has already started under the Bank's ERIPTA project and through programs funded by other donors.

25. In the infrastructural sectors, the Bank is assisting the authorities in the commercialization of ZESCO. Analytical work aims at identifying the main bottlenecks with 
regard to competition and effective service delivery. The policy recommendations and institutional changes would be supported through adjustment operations. In particular, the ongoing and proposed investment projects (Road Rehabilitation and Maintenance Project and the Water Sector Reform Project) would provide the financial resources needed for investments. Zambia will also benefit from the Southern African Power Pool Project.

26. Environmental concerns are addressed as part of each individual project as laid down in the Bank's safeguard policies. They are also addressed through the Bank's environmental project in the Copperbelt, which will assist Government with the cleanup of several hazardous sites in the Copperbelt and Kabwe area. The proposed SEED project will assist the Government in making environmentally sustainable tourism in protected areas.

\section{C.2. Areas in which the World Bank leads and its analysis serves as input into the IMF program}

27. The Bank leads the dialogue on institutional dimensions of public expenditure management and financial accountability as well as public sector reform and restructuring and privatization. The Bank program will support these areas through a combination of lending and analytical work. In the area of institutional dimensions of public expenditure management and financial accountability, the Bank's long-term objective is to encourage a more transparent and accountable public sector by improving budget management, procurement systems, and the accountability for public funds. The public sector reform and restructuring agenda centers on instilling a more productive and efficient public service. To achieve this goal, reform efforts aim at promoting a more qualified and motivated civil service, developing a strategic management orientation in line ministries, improving policy coordination and implementation, and lastly, strengthening local government's capacity to participate in the development process.

28. While the Bank has taken the lead in privatization, the IMF has also a strong interest in these areas since many of the reforms are critical to achieving macroeconomic stabilization and enhancing Zambia's growth prospects. Accordingly, there is a high degree of consultation and coordination between the two institutions on these matters.

\section{C.3. Areas of shared responsibility}

29. The Fund and the Bank are working jointly in the following five areas (i) trade policy; (ii) financial sector reform; (iii) debt management and domestic debt market development; (iv) PRSP monitoring and evaluation; and (v) assessment of progress towards the completion point under the HIPC Initiative and preparation of a debt sustainability analysis.

30. In the area of trade policy, the Bank, with collaboration from other partners, will undertake an integrated framework exercise. The Fund and the Bank jointly reviewed the Financial Sector Development Plan (FSDP). Follow ups on the FSAP and the FSDP will guide the Bank's policy dialogue and interventions in the financial sector in close 
collaboration with the IMF. The PRGF and the Economic Management and Growth Credit assist the Government on the resolution of three state-owned Non-Bank Financial Institutions.

31. Assistance in the areas of central government debt management and domestic debt market development will focus on reducing vulnerability and the cost of and the need for domestic financing. This work will draw from the Guidelines for Public Debt Management (published in March 2001) and the Handbook on Developing Government Bond Markets (published in July 2001). It will also benefit from an accompanying document to the Guidelines (prepared by the World Bank and the IMF), containing case studies from a range of countries at different stages of economic and financial development. The Bank, through its Treasury department, has under taken a debt management assessment and currently assists the Government in putting the recommended institutional changes in place.

32. The Fund and the Bank are working closely to review progress with Zambia's PRSP. The Bank and the Fund jointly prepared the Joint Staff Assessment (JSA) of the full PRSP (published in May 2002), the JSA of the first annual progress report (May 2004), and the Joint Staff Advisory Note on the second annual progress report (March 2005). Zambia reached its decision point in December 2000. Finally, the Fund and the Bank jointly prepared the HIPC-AAP document, monitored progress towards the completion point under the Enhanced HIPC Initiative, and prepared a debt sustainability analysis.

\section{C.4. Areas in which the Fund leads and its analysis serves as input into the World Bank program}

33. The Fund leads the dialogue on macroeconomic framework, in particular with regard to fiscal policy, advising the Government on the overall envelope for public expenditures, tax policy, as well as monetary and exchange rate policy. In these areas the Bank takes into account the policy recommendations of the Fund and ensures that its own policy advice, embedded in its adjustment operation (the Economic Management and Growth Credit), is consistent. 


\section{Table 2: IMF-World Bank Collaboration in Zambia}

\begin{tabular}{|c|c|c|c|}
\hline CAS Component & $\begin{array}{c}\text { Specialized advice } \\
\text { from Fund }\end{array}$ & $\begin{array}{l}\text { Specialized advice } \\
\text { from World Bank }\end{array}$ & Key Instruments \\
\hline \multicolumn{4}{|c|}{ Goal: A growth conducive macro economic environment } \\
\hline $\begin{array}{l}\text { Subject: Economic } \\
\text { Framework/ } \\
\text { Management }\end{array}$ & $\begin{array}{l}\text { Monetary policy; } \\
\text { Financial sector } \\
\text { reforms, exchange } \\
\text { rate, fiscal policy } \\
\text { and wage bill, debt } \\
\text { management, } \\
\text { balance of } \\
\text { payments, } \\
\text { economic statistics }\end{array}$ & $\begin{array}{l}\text { Debt management; } \\
\text { payroll management; } \\
\text { Financial sector } \\
\text { reforms }\end{array}$ & $\begin{array}{l}\text { IMF: PRGF performance criteria and } \\
\text { benchmarks on key monetary and fiscal } \\
\text { targets and structural reforms } \\
\text { Bank: } \\
\text { Ongoing portfolio: Public Sector Capacity } \\
\text { Building Project (PSCAP) (FY00); EMGC } \\
\text { (FY05) } \\
\text { New Lending: EMGC II (DPL) (FY06) } \\
\underline{\text { ESW: Debt Management Assessment }} \\
\text { Jointly: JSAN on PRSP progress report and } \\
\text { debt sustainability analysis }\end{array}$ \\
\hline \multicolumn{4}{|c|}{ Goal: A diversified and export oriented economy } \\
\hline $\begin{array}{l}\text { Subject: Private } \\
\text { sector development; } \\
\text { Trade and export } \\
\text { diversification }\end{array}$ & $\begin{array}{l}\text { Financial sector } \\
\text { reform; trade } \\
\text { policy; exchange } \\
\text { rate policy }\end{array}$ & $\begin{array}{l}\text { Economic growth, } \\
\text { investment climate } \\
\text { for private sector } \\
\text { (infrastructure } \\
\text { services, reforms of } \\
\text { financial sector and } \\
\text { pension funds, power } \\
\text { and } \\
\text { telecommunication } \\
\text { sector reforms, } \\
\text { vocational training), } \\
\text { trade policy for } \\
\text { export } \\
\text { diversification, rural } \\
\text { development, } \\
\text { environmental } \\
\text { regulations for the } \\
\text { mining sector }\end{array}$ & $\begin{array}{l}\text { IMF: PRGF performance criteria and } \\
\text { benchmarks on key monetary and fiscal } \\
\text { targets and structural reforms. } \\
\text { Bank: } \\
\text { On-going Portfolio: ERIPTA, Copperbelt } \\
\text { Environment Program, ROADSIP, Mine } \\
\text { Township Services Project (FY00), Regional } \\
\text { Trade Facilitation Project (FY00), Railways } \\
\text { Restructuring Project (FY00), TEVET (FY00) } \\
\text { Zambia Power Rehabilitation, SEED (FY05), } \\
\text { EMGC (FY05). } \\
\text { New Lending: EMGC II (DPL-FY06), EMGC } \\
\text { III (FY07), PMSP (FY05), Increased Access } \\
\text { to Electricity, South Africa Power Pool. } \\
\text { ESW: Country Economic Memorandum } \\
\text { (FY04), Investment Climate Assessment } \\
\text { (FY04), ICT Options Study (FY06), Energy } \\
\text { Services Delivery. } \\
\text { Jointly: FSAP (FY02) and follow up, JSAN } \\
\text { of PRSP progress report }\end{array}$ \\
\hline \multicolumn{4}{|c|}{ Goal: Better health and long-term survival, with particular focus on at risk and vulnerable groups } \\
\hline Subject: Health & & $\begin{array}{l}\text { Health Sector } \\
\text { Expenditure } \\
\text { Management. } \\
\text { Provision of } \\
\text { sustainable water } \\
\text { services in provinces } \\
\text { and districts }\end{array}$ & $\begin{array}{l}\text { Bank: } \\
\text { On-going Portfolio: ZANARA (FY03), } \\
\text { ZAMSIF (FY00), Mine Townships Project } \\
\text { (FY00) } \\
\text { New Lending: PRSC Health (FY07) (high } \\
\text { case only) } \\
\text { ESW: Health Sector PER (FY06), Strategic } \\
\text { Country Gender Assessment (FY04) }\end{array}$ \\
\hline
\end{tabular}




\begin{tabular}{|c|c|c|c|}
\hline \multicolumn{4}{|c|}{ Goal: Reverse the spread of HIV/AIDS: decrease prevalence among } \\
\hline Subject: HIV/AIDS & & $\begin{array}{l}\text { Behavioral change; } \\
\text { education; } \\
\text { prevention, care and } \\
\text { risk mitigation } \\
\text { services. }\end{array}$ & $\begin{array}{l}\text { Bank: } \\
\text { On-going Portfolio: ZANARA (FY03), } \\
\text { ZAMSIF (FY00), Aids Component } \\
\text { BESSIP(FY00) } \\
\text { New Lending: HIV/AIDS Corridor Project } \\
\text { (Sub-regional project) (FY07) } \\
\text { ESW: Social Safety Nets and Protection } \\
\text { Strategy Note (FY04), Strategic Country } \\
\text { Gender Assessment (FY04) }\end{array}$ \\
\hline \multicolumn{4}{|c|}{ Goal: Better educated populace, with relevant job skills as well as academic training } \\
\hline Subject: Education & & $\begin{array}{l}\text { Provision and quality } \\
\text { of primary education }\end{array}$ & $\begin{array}{l}\text { Bank: } \\
\text { On-going Portfolio: BESSIP I } \\
\text { (SWAP)(FY99), TEVET(FY01) } \\
\text { ZAMSIF (FY00) } \\
\text { New Lending: PRSC (education) FY06 (high } \\
\text { case only) } \\
\text { ESW: Education Sector PER(FY05) } \\
\end{array}$ \\
\hline \multicolumn{4}{|c|}{ Goal: Improved living conditions for poor/vulnerable households in drought prone areas } \\
\hline $\begin{array}{l}\text { Subject: Social } \\
\text { Protection/ Risk } \\
\text { Mitigation }\end{array}$ & & $\begin{array}{l}\text { Social protection and } \\
\text { risk management } \\
\text { systems }\end{array}$ & $\begin{array}{l}\text { IMF: PRGF } \\
\text { Bank: } \\
\text { Ongoing Portfolio: Emergency Drought } \\
\text { Recovery Project, SEED } \\
\text { New Lending: Health/Nutrition Project, } \\
\text { Agriculture Project. } \\
\text { ESW: Poverty and Vulnerability Assessment, } \\
\text { Strategic Country Gender Assessment, Social } \\
\text { Safety Nets and Social Protection Strategy } \\
\text { Note, Health Sector Review }\end{array}$ \\
\hline \multicolumn{4}{|c|}{ Goal: Good governance and public sector management } \\
\hline $\begin{array}{l}\text { Subject: Public } \\
\text { Expenditure }\end{array}$ & $\begin{array}{l}\text { Medium-term } \\
\text { budget framework, } \\
\text { tax policy and } \\
\text { administration. } \\
\text { Arrears and } \\
\text { commitment } \\
\text { control }\end{array}$ & $\begin{array}{l}\text { Public expenditure } \\
\text { analysis, } \\
\text { Capacity building in } \\
\text { financial } \\
\text { management and } \\
\text { accountability }\end{array}$ & $\begin{array}{l}\text { IMF: PRGF measures to put in place the } \\
\text { IFMIS and to ensure timely reporting on } \\
\text { budget execution } \\
\text { Bank: } \\
\text { On-going Portfolio: PSCAP I (FY00), EMGC } \\
\text { (FY05) } \\
\text { New Lending: PSMP(FY05); EMGC II } \\
\text { (FY06); EMGC III (FY07) } \\
\text { ESW: PEMFAR (FY03) } \\
\text { Jointly: HIPC AAP expenditure tracking } \\
\text { assessment (FY04) }\end{array}$ \\
\hline \multicolumn{4}{|c|}{ Goal: A productive and efficient public service } \\
\hline $\begin{array}{l}\text { Subject: Public } \\
\text { Sector Reform and } \\
\text { Restructuring }\end{array}$ & & $\begin{array}{l}\text { Pay reform, public } \\
\text { sector restructuring }\end{array}$ & $\begin{array}{l}\text { Bank: } \\
\text { On-going Portfolio: PSCAP I; EMGC (FY05) } \\
\text { New Lending: PSMP (FY05), EMGC II } \\
\text { (FY06), EMGC III (FY07) } \\
\text { ESW: PEMFAR(FY03), Governance Survey }\end{array}$ \\
\hline
\end{tabular}

Questions may be referred to Jos Verbeek (202-473-5539). 


\section{Statement by the IMF Staff Representative April 8, 2005}

This statement provides additional information that has become available since the issuance of the staff report. Recent developments were broadly in line with the program and do not alter the thrust of the staff appraisal.

- In March, the 12-month rate of inflation stood at 17.4 percent (in line with program assumptions), down from 18.7 percent in February, reflecting a leveling-off of food prices. Low rainfall in some regions of the country may reduce the maize harvest in coming months, but a firm estimate will not be available until the crop forecasting survey is completed in late April or early May. Existing maize stocks amount to about 10 percent of annual consumption. As a precautionary measure, the government has temporarily banned the export of maize.

- Preliminary data through February indicate that government revenues were about 1 percent below authorities' monthly target, which is consistent with program projections for end-March. Net domestic financing of the government was similarly also in line with the authorities' end-February ceiling after applying program adjustors.

- Net domestic assets of the Bank of Zambia were held within the authorities' monthly ceiling for end February and gross reserves of the Bank of Zambia exceeded the adjusted floor by comfortable margins. In line with the program, reserve and broad money growth moderated substantially, as the 12-month growth rates dropped to $15 \frac{1}{2}$ percent and 221/2 percent, respectively, in February. The average yield on treasury bills eased slightly in March to 18.0 percent, from 18.3 percent in February while the kwacha appreciated against the U.S. dollar by 0.5 percent in nominal terms.

- On March 29, cabinet approved the proposal to repeal sections of the Building Societies Act, the National Savings and Credit Bank Act, and the Development Bank of Zambia Amendment Act which were in conflict with the Banking and Financial Services Act. The written notification of this measure received by staff on March 30 satisfies the prior action for the Board discussion of the PRGF review.

- On March 31, the Zambia Privatization Agency (ZPA) advertised an invitation for bids for the sale of a 49 percent share with management rights of the Zambia National Commercial Bank. The staff welcomes this action, which has restarted the privatization process and could soon lead to the issuance of international bidding documents as called for by the HIPC trigger on which a waiver is recommended. The ZPA has established a deadline for submitting bids of June 30, 2005. In addition, since the issuance of the invitation for bids fulfilled a proposed performance criterion for May 2005, a revised decision removing this performance criterion has been circulated. 
- Parliament approved the 2005 budget on March 30. The final budget is in line with the framework under the PRGF-supported program, including a ceiling on government domestic financing of 1.6 percent of GDP. Total revenues and expenditures are unchanged from the original budget proposal. The budget includes a minor reallocation of spending (of 0.1 percent of GDP) to grants to local governments with offsetting cuts in various administrative and capital expenditures and the contingency.

- The contract for the installation of the integrated financial management and information system (IFMIS) has yet to be finalized. The World Bank's Legal Department recommended changes to a proposal for a joint venture arrangement between the contractor and its former parent company, in order to comply with the original prequalification conditions for bids. At this point, the authorities and the contractor have not indicated whether these recommendations are acceptable.

- The authorities have given their consent to the publication of the staff report and the Completion Point Document for the Enhanced HIPC Initiative. 
April 8, 2005

\section{IMF Executive Board Completes Second Review Under Zambia's PRGF Arrangement and Approves US\$8.3 Million Disbursement}

The Executive Board of the International Monetary Fund (IMF) today completed the second review of Zambia's economic performance under a three-year Poverty Reduction and Growth Facility (PRGF) arrangement, which was approved on June 16, 2004 (see Press Release No. $\underline{04 / 117}$ ). The Board also approved waivers for the nonobservance of the continuous quantitative performance criterion on the nonaccumulation of new external arrears and the end-February 2005 structural performance criterion on cabinet approval of proposals to repeal sections of various acts that are in conflict with the Banking and Financial Services Act.

The completion of the review enables the release of a further SDR 5.5 million (about US\$8.3 million), which will bring the total amount drawn under the arrangement to about SDR 170.6 million (about US\$256.7 million).

Following the Executive Board discussion, Mr. Takatoshi Kato, Deputy Managing Director and Acting Chair, said:

“Zambia's economic performance continued to strengthen in 2004, and over the last five years annual growth averaged nearly $4 \frac{1}{2}$ percent. This performance is particularly encouraging against the backdrop of the economic decline in the preceding two decades. However, growth is still not strong enough to make large inroads into widespread poverty, and inflation is high, even though progress was made in this area in 2004 despite increases in fuel prices.

"The implementation of the large fiscal adjustment envisaged under the PRGF-supported program, which embraced politically difficult spending restraint, allowed for a sharp decline in government domestic borrowing and halted an unsustainable rise in domestic debt. The wage bill was held to less than 8 percent of GDP and, for the first time, priority poverty-reducing programs (PRPs) were fully funded. The 2005 budget framework seeks to consolidate these gains to contain domestic borrowing while allowing for increased spending on priority PRPs, including the hiring of a substantial number of additional teachers and frontline health workers.

"Lower government borrowing allowed for a large increase in bank credit to the private sector in 2004, which had a positive impact on investment and the prospects for future economic growth. Moreover, the authorities are taking active steps to deepen financial intermediation through the privatization of the Zambia National Commercial Bank, resolution of some problem state-owned 
nonbank financial institutions, and strengthening of the supervisory role of the Bank of Zambia. In addition, rapid progress in implementing the private sector development initiative would further enhance economic growth.

"Zambia's attainment of the completion point under the enhanced Initiative for Heavily Indebted Poor Countries (HIPC) will greatly improve its debt sustainability position and provide greater resources for poverty-reducing priorities. The authorities are strongly encouraged to quickly reach agreements with all creditors on the delivery of HIPC assistance. In addition, timely implementation of the comprehensive work plan to strengthen public expenditure management, along with further progress toward macroeconomic stabilization, could lead to a scaling up of donor support for Zambia's efforts to reduce poverty. In this connection, it is important that the authorities complete the updating of Zambia's Poverty Reduction Strategy Paper/ National Development Plan before the year's end," Mr. Kato said.

The PRGF is the IMF's concessional facility for low-income countries. It is intended that PRGFsupported programs are based on country-owned poverty reduction strategies adopted in a participatory process involving civil society and development partners and articulated in a Poverty Reduction Strategy Paper (PRSP). This is intended to ensure that PRGF-supported programs are consistent with a comprehensive framework for macroeconomic, structural, and social policies to foster growth and reduce poverty. PRGF loans carry an annual interest rate of 0.5 percent and are repayable over 10 years with a $5 \frac{1}{2}$-year grace period on principal payments. 


\section{Statement by Peter Ngumbullu, Executive Director for Zambia and John Steytler, Advisor to Executive Director April 8, 2005}

1. On behalf of the Zambian authorities, we thank staff and Fund management for the constructive engagement in Zambia and for the positive assessment that Zambia has satisfactorily met the requirements for reaching the completion point under the Enhanced Initiative for Heavily Indebted Poor Countries (HIPC). The successful implementation of the economic program supported by the PRFG arrangement, along with the implementation of the PRSP and the significant progress made in meeting the completion point triggers, have been the basis for Zambia's attainment of these achievements. The authorities have implemented almost all the triggers, with only three out of fifteen which have not been fully implemented; however, substantial progress has been made towards reaching them. The authorities view the staff report as fair and balanced and would like to reiterate their commitment to the objectives of the economic program and the strategy for reaching them as outlined in the Memorandum of Economic and Financial Policies (MEFP) attached to their Letter of Intent.

\section{Recent Economic Developments}

2. Zambia's economy continued to expand solidly for the fifth consecutive year, fueled by agriculture, mining and construction. Real GDP growth is estimated to have reached 5.0 percent in 2004, while inflation, despite a sharp increase in fuel oil prices during the year, was contained at 17.5 percent. Instrumental to this good performance were the structural reforms and improved macroeconomic management, particularly in the fiscal area implemented in previous years.

3. Fiscal discipline remains the cornerstone of Zambia's macroeconomic stabilization effort. In this regard, fiscal policy implementation in 2004 was broadly in line with the program. Total spending was 1.0 percent of GDP less than programmed, while tax collection strengthened. Improved fiscal discipline, and the delivery of external budget support in line with program expectations, allowed for a sharp decline in government's domestic borrowing from over 5.0 percent of GDP in 2003 to less than 1 percent of GDP in 2004. In addition, the authorities strengthened expenditure controls, including the wage bill, which was restrained at 7.8 percent of GDP as budgeted.

4. During 2004, despite efforts to tighten liquidity conditions, monetary growth surged at the end of the year mostly due to unanticipated large donor disbursements and increased foreign currency deposits by corporations. As a result, reserve money increased to 21 percent at end-year, while broad money reached 30 percent. Notwithstanding these developments, commercial banks' lending rates, supported by fiscal discipline stabilized at around 37 percent in the second half of 2004, while interest rates on treasury bills rose in mid-year and stabilized in the final quarter at about 18 percent. Credit to the private sector increased strongly in 2004, driven by large demand for financing in agriculture and manufacturing sectors. 
5. The external current account position improved in 2004, benefiting from increases in earnings from copper and other metal exports due to higher prices and increased production. Most notably, growth of non-traditional exports, including cotton, lint and tobacco, exceeded projections and contributed to strengthening Zambia's external position. Gross international reserves exceeded program target by US\$35 million, reaching US $\$ 222$ million by year-end, an import coverage of 1.2 months of goods and services. The exchange rate has remained relatively stable in nominal terms, although for the whole year the kwacha has appreciated in real terms by about 8 percent.

6. The authorities have finalized their second PRSP Implementation Progress Report reviewing the period of July 2003 to June 2004 and policies for 2005. The authorities view the joint staff advisory note as a balanced assessment of the progress being made in the implementation of the PRSP and have reiterated their commitment to the policies and strategy in the PRSP.

7. Performance under the program continued to be strong, as most of the performance criteria and policy benchmarks set under the program for the period up to end-December 2004 were observed, several of them with large margins. The authorities are, nevertheless, requesting the Board to grant waivers for the nonobservance of two performance criteria. However, the two criteria on external arrears payments and strengthening of the Bank of Zambia's supervision capacity have in the mean time been met.

\section{Medium-Term Strategy and Program for 2005}

8. Notwithstanding the encouraging economic results, the Zambian authorities are fully committed to the reform agenda. The medium-term strategy, embedded in the government's PRSP, aims at bolstering the conditions for sustained high levels of private sector led economic growth needed to reduce poverty and achieve the MDGs. Key macroeconomic objectives include real GDP growth of at least five percent a year over the medium term, with annual inflation falling to single digits by 2007 . The authorities remain committed to increasing poverty-reducing spending by at least 1.6 percent of GDP between 2004 and 2007, while gradually reducing government domestic borrowing over the medium term.

9. The economic policy agenda for 2005 remains essentially unchanged from that presented in the MEFP during the First Review under the PRGF arrangement. The policies set forth in the current MEFP continue to be adequate to achieve the objectives of the program, which envisage a real GDP growth of 5.0 percent and a further deceleration of inflation to 15 percent, with a target of reaching single digits by 2007 . However, due to the sharp increase in monetary aggregates in late 2004, some adjustments to the monetary program were necessary to achieve the objective of lowering inflation by end-2005. In addition, new structural measures have been introduced in the areas of public expenditure management and financial sector development.

10. The authorities are determined to enhance the fiscal consolidation process, while ensuring increased effectiveness of public spending, promoting strong economic growth and meeting poverty reduction goals. The budget for 2005 , which was approved by parliament on 
March 30, this year is consistent with the framework of the first review and limits government's domestic borrowing to 1.6 percent of GDP. It also includes measures to ease the burden of direct taxation, while broadening the tax base to raise revenues. Overall, the tax policy measures are expected to raise revenues by 0.1 percent of GDP. On the expenditure side, the authorities recognize that containing the wage bill is critical for maintaining macroeconomic stability and fiscal targets. The budget limits the wage bill to 8.1 percent of GDP, a slight augmentation as compared to 7.8 percent of GDP achieved in 2004. This increase will mostly accommodate the hiring of additional teachers and health workers. The overall deficit, including grants, is projected at 2.5 percent of GDP.

11. Monetary policy is geared towards reducing inflation and strengthening international reserves. In addition, the monetary program for 2005 was tightened to reverse the unanticipated increase of monetary aggregates at end of 2004. Growth in reserve money will be limited to 10.5 percent, while growth of broad money will be contained at 14.8 percent. However, the program will continue to accommodate an expansion of credit to the private sector in real terms as commercial banks are expected to make good use of the extraordinary buildup of foreign assets at the end of last year.

12. The external outlook for 2005 is expected to be favorable, despite the oil import bill which is likely to remain high. In this connection, with the continued recovery in export earnings, the external current account deficit, including grants, is expected to narrow further to 3.1 percent of GDP in 2005.

13. The authorities are committed to stepping up the structural reform agenda during 2005, which they see as providing critical support to macroeconomic stability and growth. The reforms will continue to focus on public expenditure management and financial accountability (PEMFA), including implementation of the integrated financial management information system (IFMIS) and on financial sector development, and improvement of the operations of Non-Bank Financial Intermediaries (NBFIs). The authorities are also committed to strengthening their capacity for debt management capacity and preparation of a public debt management reform plan. With regard to private sector development, they intend to actively collaborate with the World Bank in designing a work program that will address existing deficiencies in this area. Special attention will also continue to be devoted to creating an enabling environment for private sector development, including supporting the implementation of the private sector development initiative (PSDI) and advancing further the commercialization strategy. In this regard, the authorities intend to conclude the sale of 49 percent of the equity in the Zambia National Commercial Bank to a strategic investor. On March 31, 2005, the Zambia Privatization Agency issued a new invitation for bids for the purchase of 49 percent equity with management control. The authorities are also proceeding with the implementation of a comprehensive commercialization strategy for ZESCO.

\section{Conclusion}

14. The Zambian authorities are grateful to the Fund and the international community for their financial and technical assistance support, which has been instrumental in enabling them meet the existing social and economic challenges in the reform agenda. The authorities 
are looking forward to the completion of this review under the PRGF arrangement and to achieving the completion point under the Enhanced HIPC Initiative. The authorities have consented to the publication of the staff papers and the Memorandum of Economic and Financial Policies (MEFP). 